Pacific

Journal of

Mathematics

NONCOMMUTATIVE DIFFERENTIALS ON POISSON-LIE GROUPS AND PRE-LIE ALGEBRAS

Shahn MaJid AND Wen-QING TaO

Volume $284 \quad$ No. 1 


\title{
NONCOMMUTATIVE DIFFERENTIALS ON POISSON-LIE GROUPS AND PRE-LIE ALGEBRAS
}

\author{
SHAHN MAJID AND WEN-QING TAO
}

\begin{abstract}
We show that the quantisation of a connected simply connected Poisson-Lie group admits a left-covariant noncommutative differential structure at lowest deformation order if and only if the dual of its Lie algebra admits a preLie algebra structure. As an example, we find a pre-Lie algebra structure underlying the standard 3-dimensional differential structure on $\mathbb{C}_{q}\left[\mathrm{SU}_{2}\right]$. At the noncommutative geometry level we show that the enveloping algebra $U(\mathfrak{m})$ of a Lie algebra $\mathfrak{m}$, viewed as quantisation of $\mathfrak{m}^{*}$, admits a connected differential exterior algebra of classical dimension if and only if $\mathfrak{m}$ admits a pre-Lie algebra structure. We give an example where $\mathfrak{m}$ is solvable and we extend the construction to tangent and cotangent spaces of Poisson-Lie groups by using bicross-sum and bosonisation of Lie bialgebras. As an example, we obtain a 6-dimensional left-covariant differential structure on the bicrossproduct quantum group $\mathbb{C}\left[\mathrm{SU}_{2}\right] \triangleleft U_{\lambda}\left(\mathrm{su}_{2}^{*}\right)$.
\end{abstract}

\section{Introduction}

It is well-known following [Drinfeld 1987] that the semiclassical objects underlying quantum groups are Poisson-Lie groups. This means a Lie group together with a Poisson bracket such that the group product is a Poisson map. The infinitesimal notion of a Poisson-Lie group is a Lie bialgebra, meaning a Lie algebra $\mathfrak{g}$ equipped with a "Lie cobracket" $\delta: \mathfrak{g} \rightarrow \mathfrak{g} \otimes \mathfrak{g}$ forming a Lie 1-cocycle and such that its adjoint is a Lie bracket on $\mathfrak{g}^{*}$. Of the many ways of thinking about quantum groups, this is a "deformation" point of view in which the coordinate algebra on a group is made noncommutative, with commutator controlled at lowest order by the Poisson bracket.

In recent years, the examples initially provided by quantum groups have led to a significant "quantum groups approach" to noncommutative differential geometry in

Majid was on leave at the Mathematical Institute, Oxford, during 2014 when this work was completed. Tao was supported by the China Scholarship Council.

MSC2010: 17D25, 58B32, 81R50.

Keywords: noncommutative geometry, quantum group, left-covariant, differential calculus, bicovariant, deformation, Poisson-Lie group, pre-Lie algebra, (co)tangent bundle, bicrossproduct, bosonisation. 
which the next layers of geometry beyond the coordinate algebra are considered, and often classified with the aid of quantum group symmetry. The most important of these is the differential structure (also known as the differential calculus) on the coordinate algebra, expressed normally as the construction of a bimodule $\Omega^{1}$ of "1-forms" over the (possibly noncommutative) coordinate algebra $A$ and a map $\mathrm{d}: A \rightarrow \Omega^{1}$ (called the exterior derivation) satisfying the Leibniz rule. Usually, $\Omega^{1}$ is required to be spanned by elements of the form $a \mathrm{~d} b$, where $a, b \in A$. This is then typically extended to a differential graded algebra (DGA) $(\Omega, \mathrm{d})$ of all degrees where $\Omega$ is formulated as a graded algebra $\Omega=\bigoplus_{i \geq 0} \Omega^{i}$ generated by $\Omega^{0}=A, \Omega^{1}$, and $\mathrm{d}$ is a degree-one map such that $\mathrm{d}^{2}=0$ and the "super-Leibniz rule" holds, namely $\mathrm{d}(\xi \eta)=(\mathrm{d} \xi) \eta+(-1)^{n} \xi \mathrm{d} \eta$ for all $\xi \in \Omega^{n}, \eta \in \Omega$. The semiclassical version of what this data means at the Poisson level is known to be a Poisson-compatible preconnection (or "Lie-Rinehart connection"; see Remark 2.2). The systematic analysis in [Beggs and Majid 2006] found, in particular, a no-go theorem proving the nonexistence of a left and right translation-covariant differential structure of classical dimension on standard quantum group coordinate algebras $\mathbb{C}_{q}[G]$ when $G$ is the connected and simply connected Lie group of a complex semisimple Lie algebra $\mathfrak{g}$. Beggs and Majid [2010] had a similar result for the nonexistence of ad-covariant differential structures of classical dimension on enveloping algebras of semisimple Lie algebras. Such results tied in with experience at the algebraic level, where one often has to go to higher-dimensional $\Omega^{1}$, and [Beggs and Majid $2006 ; 2010]$ also provided an alternative, namely to consider nonassociative exterior algebras corresponding to preconnections with curvature. This has been taken up further in [Beggs and Majid 2014b].

The present paper revisits the analysis focussing more clearly on the Lie algebraic structure. For left-covariant differentials on a connected and simply connected Poisson-Lie group, we find (Corollary 4.2) that the semiclassical data exists if and only if the dual Lie algebra $\mathfrak{g}^{*}$ of the Lie algebra $\mathfrak{g}$ admits a so-called pre-Lie structure $\Xi: \mathfrak{g}^{*} \otimes \mathfrak{g}^{*} \rightarrow \mathfrak{g}^{*}$. Here a pre-Lie structure is a product obeying certain axioms such that the commutator is a Lie algebra, such objects also being called leftsymmetric or Vinberg algebras; see [Cartier 2009] and [Burde 2006] for two reviews. Our result has no contradiction to $\mathfrak{g}$ being semisimple and includes quantum groups such as $\mathbb{C}_{q}\left[\mathrm{SU}_{2}\right]$, where we exhibit the pre-Lie structure that corresponds to its known 3-dimensional calculus in [Woronowicz 1989].

Even better, the duals $\mathfrak{g}^{*}$ for all the quantum groups $\mathbb{C}_{q}[G]$ are known to be solvable [Majid 1990a] and it may be that all solvable Lie algebras admit pre-Lie algebra structures, a question posed by Milnor; see [Burde 2006]. This suggests for the first time a systematic route to the construction of left-covariant differential calculi for all $\mathbb{C}_{q}[G]$, currently an unsolved problem. We build on the initial analysis of this example in [Beggs and Majid 2006]. Next, for the calculus to be both left 
and right covariant (i.e., bicovariant), we find an additional condition (4-6) on $\Xi$ which we relate to infinitesimal or Lie-crossed modules with the coadjoint action; see Theorems 3.1 and 4.1 .

The paper also covers in detail the important case of the enveloping algebra $U(\mathfrak{m})$ of a Lie algebra $\mathfrak{m}$, viewed as a quantisation of $\mathfrak{m}^{*}$. This is a Hopf algebra so, trivially, a quantum group, and our theory applies with $\mathfrak{g}=\mathfrak{m}^{*}$ an abelian Poisson-Lie group with its Kirillov-Kostant Poisson bracket. In fact our result in this example turns out to extend canonically to all orders in deformation theory, not just the lowest semiclassical order. We show (Proposition 4.4) that $U(\mathfrak{m})$ admits a connected bicovariant differential exterior algebra of classical dimension if and only if $\mathfrak{m}$ admits a pre-Lie structure. The proof builds on results in [Majid and Tao 2015b]. We do not require ad-invariance but the result excludes the case that $\mathfrak{m}$ is semisimple since semisimple Lie algebras do not admit pre-Lie structures [Burde 1994]. The $\mathfrak{m}$ that are allowed do, however, include solvable Lie algebras of the form $\left[x_{i}, t\right]=x_{i}$, which have been extensively discussed for the structure of "quantum spacetime" (here $x_{i}$ and $t$ are now viewed as space and time coordinates, respectively), most recently in [Beggs and Majid 2014a]. In the 2-dimensional case we use the known classification of 2-dimensional pre-Lie structures over $\mathbb{C}$ in [Burde 1998] to classify all possible left-covariant differential structures of classical dimension. This includes the standard calculus previously used in [Beggs and Majid 2014a] as well as some other differential calculi in the physics literature [Meljanac et al. 2012]. The 4-dimensional case and its consequences for quantum gravity are explored in our related paper [Majid and Tao 2015a].

We then apply our theory to the quantisation of the tangent bundle and cotangent bundle of a Poisson-Lie group. In Section 5, we recall the use of the Lie bialgebra $\mathfrak{g}$ of a Poisson-Lie group $G$ to construct the tangent bundle as a bicrossproduct of Poisson-Lie groups and its associated "bicross-sum" of Lie bialgebras [Majid 1995]. Our results (see Theorem 5.6) then suggest a full differential structure, not only at semiclassical level, on the associated bicrossproduct quantum groups $\mathbb{C}[G] \bowtie U_{\lambda}\left(\mathfrak{g}^{*}\right)$ in [Majid 1990a; 1990b; 1995]. We prove this in Proposition 5.7 and give $\mathbb{C}\left[\mathrm{SU}_{2}\right] \triangleleft U_{\lambda}\left(\mathrm{su}_{2}^{*}\right)$ in detail. Indeed, these bicrossproduct quantum groups were exactly conceived in the 1980s as quantum tangent spaces of Lie groups. In Section 6 , we use a pre-Lie structure on $\mathfrak{g}^{*}$ to make $\mathfrak{g}$ into a braided-Lie biaglebra [Majid 2000] (see Lemma 6.1). The Lie bialgebra of the cotangent bundle becomes a "bosonisation" in the sense of [Majid 2000] and we construct in some cases a natural preconnection for the semiclassical differential calculus. As before, we cover abelian Lie groups with the Kirillov-Kostant Poisson bracket and a restricted class of quasitriangular Poisson-Lie groups as examples.

Most of the work in the paper is at the semiclassical level but occasionally we have results about differentials at the Hopf algebra level as in [Woronowicz 1989], 
building on [Majid and Tao 2015b]. We recall that a Hopf algebra $A$ is an algebra equipped with a compatible coalgebra and an "antipode" $S$ in the role of inverse. We denote the coproduct $\Delta: A \rightarrow A \otimes A$ by the Sweedler notation $\Delta a=a_{(1)} \otimes a_{(2)}$. A differential calculus $\left(\Omega^{1}, \mathrm{~d}\right)$ on a Hopf algebra is called left-covariant if $\Omega^{1}$ is a left $A$-comodule with coaction $\Delta_{L}: \Omega^{1} \rightarrow A \otimes \Omega^{1}$ satisfying $\Delta_{L}(a \mathrm{~d} b)=$ $a_{(1)} b_{(1)} \otimes a_{(2)} \mathrm{d} b_{(2)}$ for all $a, b \in A$. Similarly for a right-covariant calculus with structure map $\Delta_{R}: \Omega^{1} \rightarrow \Omega^{1} \otimes A$ satisfying $\Delta_{R}(a \mathrm{~d} b)=a_{(1)} \mathrm{d} b_{(1)} \otimes a_{(2)} b_{(2)}$. A calculus is bicovariant if it is both left- and right-covariant. A left-covariant calculus can always be put in the form $\Omega^{1}=A \otimes \Lambda^{1}$ as a left $A$-module, where $\Lambda^{1}$ is the space of invariants under the left coaction, and in the bicovariant case extends canonically to a differential graded algebra $\Omega$ [Woronowicz 1989].

\section{Preliminaries}

2A. Deformation of noncommutative differentials. We follow the setting given in [Beggs and Majid 2006]. Let $M$ be a smooth manifold and consider the deformation of the coordinate algebra $C^{\infty}(M)$ by replacing the usual commutative point-wise multiplication (usually omitted) with a new multiplication $\bullet$ of the form $a \bullet b=$ $a b+O(\lambda)$ for all $a, b \in C^{\infty}(M)$. The noncommutativity of the new product can be expressed in a bracket $\{\}:, C^{\infty}(M) \otimes C^{\infty}(M) \rightarrow C^{\infty}(M)$ defined by $[a, b]_{\bullet}=a \bullet b-b \bullet a=\lambda\{a, b\}+O\left(\lambda^{2}\right)$. We assume that we are working in a deformation setting where we can equate order by order in $\lambda$. Then it is well-known that the new product $\bullet$ is associative up to order $O\left(\lambda^{2}\right)$ if and only if the bracket $\{$, is a Poisson bracket. We denote the associated bivector by $\pi$, so $\{a, b\}=\pi(\mathrm{d} a, \mathrm{~d} b)$.

In the same spirit, however, one can likewise consider the deformation of differential forms. The $n$-forms $\Omega^{n}(M)$ and exterior algebra $\Omega(M)$ are identified with their classical counterparts as vector spaces. But now $\Omega^{1}(M)$ is equipped with new left/right actions $a \bullet \tau=a \tau+O(\lambda)$ and $\tau \bullet a=\tau a+O(\lambda)$. The deformed derivation $\mathrm{d}_{\bullet}: \Omega^{n}(M) \rightarrow \Omega^{n+1}(M)$ is of the form $\mathrm{d}_{\boldsymbol{\bullet}} a=\mathrm{d} a+O(\lambda)$. Define a linear map $\gamma: C^{\infty}(M) \otimes \Omega^{1}(M) \rightarrow \Omega^{1}(M)$ by

$$
a \cdot \tau-\tau \bullet a=[a, \tau]_{\bullet}=\lambda \gamma(a, \tau)+O\left(\lambda^{2}\right) .
$$

It was shown in [Hawkins 2004; Beggs and Majid 2006] that for $\Omega^{1}(M)$ with new left/right actions to be a $\left(C^{\infty}(M), \bullet\right)$-bimodule up to order $O\left(\lambda^{2}\right)$ requires the associated map $\gamma$ to satisfy

$$
\begin{aligned}
& \gamma(a b, \tau)=\gamma(a, \tau) b+a \gamma(b, \tau), \\
& \gamma(a, b \tau)=b \gamma(a, \tau)+\{a, b\} \tau .
\end{aligned}
$$

If $\mathrm{d}_{0}$ is a derivation up to order $O\left(\lambda^{2}\right)$, then $\gamma$ should also satisfy

$$
\mathrm{d}\{a, b\}=\gamma(a, \mathrm{~d} b)-\gamma(b, \mathrm{~d} a),
$$


where $\mathrm{d}: C^{\infty}(M) \rightarrow \Omega^{1}(M)$ is the usual exterior derivation.

Definition 2.1. Any map $\gamma: C^{\infty}(M) \otimes \Omega^{1}(M) \rightarrow \Omega^{1}(M)$ satisfying (2-1) and (2-2) is called a preconnection on $M$. A preconnection $\gamma$ is said to be Poisson-compatible if (2-3) also holds.

Such preconnections can arise by pullback along the map that associates a Hamiltonian vector fields $\hat{a}=\{a,-\}$ to a function $a \in C^{\infty}(M)$, i.e., $\gamma(a,-)=\nabla_{\hat{a}}$ for a covariant derivative defined at least along Hamiltonian vector fields, in which case the remaining (2-3) appears as a constraint on its torsion. From the analysis above, we see that a Poisson-compatible preconnection controls the noncommutativity of functions and 1-forms, and thus plays a vital role in deforming a differential graded algebra $\Omega(M)$ at lowest order, parallel to the Poisson bracket for $C^{\infty}(M)$ at lowest order.

Remark 2.2. As pointed out by the referee, a Poisson-compatible preconnection in Definition 2.1 can be seen as an example of a Lie-Rinehart connection; cf. [Huebschmann 1990]. If $M$ is a Poisson manifold then the pair $\left(C^{\infty}(M), \Omega^{1}(M)\right)$ forms a Lie-Rinehart algebra with $\Omega^{1}(M)$ a Lie algebra by $[\mathrm{d} a, \mathrm{~d} b]=\mathrm{d}\{a, b\}$ for all $a, b \in C^{\infty}(M)$, where $\left(\Omega^{1}(M),[],\right)$ acts on $C^{\infty}(M)$ by $(\mathrm{d} a) \triangleright b=\pi(\mathrm{d} a, \mathrm{~d} b)=\{a, b\}$ for all $a, b \in C^{\infty}(M)$. In this context we can consider a Poisson-compatible preconnection as a covariant derivative $\nabla_{\eta}$ along 1-forms $\eta \in \Omega^{1}$ by $\nabla_{\mathrm{d} a}=\gamma(a,-)$ extended $C^{\infty}(M)$-linearly, i.e., a Lie-Rinehart connection in this context. Here (2-2) appears as the connection property $\nabla_{\eta}(a \tau)=\pi(\eta, \mathrm{d} a) \tau+a \nabla_{\eta} \tau$ while (2-3) appears as the further property $[\eta, \tau]=\nabla_{\eta} \tau-\nabla_{\tau} \eta$ for all $\eta, \tau \in \Omega^{1}(M)$.

2B. Poisson-Lie groups and Lie bialgebras. Throughout the paper, we mainly work over a Poisson-Lie group $G$ and its Lie bialgebra $\mathfrak{g}$. By definition, the Poisson bracket $\{\}:, C^{\infty}(G) \otimes C^{\infty}(G) \rightarrow C^{\infty}(G)$ is determined uniquely by a so-called Poisson bivector $\pi=\pi^{(1)} \otimes \pi^{(2)}$, i.e., $\{a, b\}=\pi^{(1)}(\mathrm{d} a) \pi^{(2)}(\mathrm{d} b)$. Then $\mathfrak{g}$ is a Lie bialgebra with Lie cobracket $\delta: \mathfrak{g} \rightarrow \mathfrak{g} \otimes \mathfrak{g}$ given by

$$
\delta(x)=\left.\frac{\mathrm{d}}{\mathrm{d} t} \pi^{(1)}(g) g^{-1} \otimes \pi^{(2)}(g) g^{-1}\right|_{t=0},
$$

where $g=\exp t x \in G$ for any $x \in \mathfrak{g}$. The map $\delta$ is a Lie 1 -cocycle with respect to the adjoint action, and extends to group 1-cocycles $D(g)=\left(R_{g^{-1}}\right)_{*} \pi(g)$ with respect to the left adjoint action and $D^{\vee}(g)=\left(L_{g^{-1}}\right)_{*} \pi(g)$ with respect to the right adjoint action, respectively. Here $D^{\vee}$ and $D$ are related by $D^{\vee}(g)=\operatorname{Ad}_{g^{-1}} D(g)$ and thus are equivalent. We recall that a left group cocycle means

$$
D(u v)=D(u)+\operatorname{Ad}_{u}(D(v)) \text { for all } u, v \in G, D(e)=0 .
$$

When $G$ is connected and simply connected, one can recover $D$ for a given $\delta$ by solving

$$
\mathrm{d} D(\tilde{x})(g)=\operatorname{Ad}_{g}(\delta x), \quad D(e)=0,
$$


where $\tilde{x}$ is the left-invariant vector field corresponding to $x \in \mathfrak{g}$. We then recover the Poisson bracket by $\pi(g)=R_{g *}(D(g))$ for all $g \in G$. These notions are due to Drinfeld and an introduction can be found in [Majid 1995].

For convenience, we recall that a left $\mathfrak{g}$-module over a Lie algebra $\mathfrak{g}$ is a vector space $V$ together with a linear map $\triangleright: \mathfrak{g} \otimes V \rightarrow V$ such that $[x, y] \triangleright v=$ $x \triangleright(y \triangleright v)-y \triangleright(x \triangleright v)$ for all $x, y \in \mathfrak{g}$ and $v \in V$. Dually, a left $\mathfrak{g}$-comodule over a Lie coalgebra $(\mathfrak{g}, \delta)$ is a vector space $V$ together with a linear map $\alpha: V \rightarrow \mathfrak{g} \otimes V$ such that $(\delta \otimes \mathrm{id}) \circ \alpha=((\mathrm{id}-\tau) \otimes \mathrm{id}) \circ(\mathrm{id} \otimes \delta) \circ \alpha$. Over a Lie bialgebra $(\mathfrak{g},[],, \delta)$, a left $\mathfrak{g}$-crossed module $(V, \triangleright, \alpha)$ is both a left $\mathfrak{g}$-module $(V, \triangleright)$ and a left $\mathfrak{g}$-comodule $(V, \alpha)$ such that

$$
\alpha(x \triangleright v)=([x,] \otimes \mathrm{id}+\mathrm{id} \otimes x \triangleright) \alpha(v)+\delta(x) \triangleright v
$$

for any $x \in \mathfrak{g}, v \in V$. When $\mathfrak{g}$ is finite-dimensional, the notion of a left $\mathfrak{g}$-crossed module is equivalent to a left $\mathfrak{g}$-module $(V, \triangleright)$ that admits a left $\mathfrak{g}^{* \text { op }}$-action $\triangleright^{\prime}$ satisfying

$$
\phi_{(1)} \triangleright^{\prime} v\left\langle\phi_{(2)}, x\right\rangle+x_{(1)} \triangleright v\left\langle\phi, x_{(2)}\right\rangle=x \triangleright\left(\phi \triangleright^{\prime} v\right)-\phi \triangleright^{\prime}(x \triangleright v)
$$

for any $x \in \mathfrak{g}, \phi \in \mathfrak{g}^{*}$ and $v \in V$, where the left $\mathfrak{g}^{* 0 \mathrm{op}}$-action $\triangleright^{\prime}$ corresponds to the left $\mathfrak{g}$-coaction $\alpha$ above via $\phi \triangleright^{\prime} v=\left\langle\phi, v^{(1)}\right\rangle v^{(2)}$ with $\alpha(v)=v^{(1)} \otimes v^{(2)}$. Therefore, a left $\mathfrak{g}$-crossed module is precisely a left $D(\mathfrak{g})$-module, where $D(\mathfrak{g})$ is the Drinfeld double of $\mathfrak{g}$; see [Majid 1995]. For brevity, we call a left $\mathfrak{g}$-module $V$ with linear map $\triangleright^{\prime}: \mathfrak{g}^{*} \otimes V \rightarrow V$ (not necessarily an action) such that (2-4) holds a left almost $\mathfrak{g}$-crossed module.

2C. Left-covariant preconnections. The algebra of functions on a Poisson-Lie group $G$ typically deforms to a noncommutative Hopf algebra $A$ and a semiclassical analysis of the covariance of a differential structure was initiated in [Beggs and Majid 2006] in terms of preconnection $\gamma$. By definition, a preconnection $\gamma$ is said to be left-covariant (right-covariant, or bicovariant) if the associated differential calculus on $\left(C^{\infty}(G), \bullet\right)$ is left-covariant (right-covariant, or bicovariant) over $\left(C^{\infty}(G), \bullet\right)$ up to $O\left(\lambda^{2}\right)$. [Beggs and Majid 2006, Lemma 4.3] gives a precise characterisation of this in terms of a map $\Xi$ as follows.

We first explain the notations used in [Beggs and Majid 2006]. We recall that there is a one-to-one correspondence between 1-forms $\Omega^{1}(G)$ and $C^{\infty}\left(G, \mathfrak{g}^{*}\right)$, the set of smooth sections of the trivial $\mathfrak{g}^{*}$ bundle. For any 1-form $\tau$, define $\tilde{\tau} \in C^{\infty}\left(G, \mathfrak{g}^{*}\right)$ by letting $\tilde{\tau}_{g}=L_{g}^{*}\left(\tau_{g}\right)$. Conversely, any $s \in C^{\infty}\left(G, \mathfrak{g}^{*}\right)$ defines an 1-form (denoted by $\left.\hat{s}\right)$ by setting $\hat{s}_{g}=L_{g^{-1}}^{*}(s(g))$. In particular, we know $\mathrm{d} a \in \Omega^{1}(G)$ and $\widetilde{\mathrm{d} a} \in C^{\infty}\left(G, \mathfrak{g}^{*}\right)$ for any $a \in C^{\infty}(G)$. Denote $\widetilde{\mathrm{d} a}$ by $\hat{L}_{a}$, then

$$
\left\langle\hat{L}_{a}(g), v\right\rangle=\langle\widetilde{\mathrm{d} a}(g), v\rangle=\left\langle L_{g}^{*}\left((\mathrm{~d} a)_{g}\right), v\right\rangle=\left\langle(\mathrm{d} a)_{g},\left(L_{g}\right)_{*} v\right\rangle=\left(L_{g}\right)_{*}(v) a,
$$


which is the directional derivation of $a$ with respect to $v \in \mathfrak{g}$ at $g$.

Using the above notations, a preconnection $\gamma$ can now be rewritten on $\mathfrak{g}^{*}$-valued functions as $\tilde{\gamma}: C^{\infty}(G) \times C^{\infty}\left(G, \mathfrak{g}^{*}\right) \rightarrow C^{\infty}\left(G, \mathfrak{g}^{*}\right)$ by letting $\left.\tilde{\gamma}(a, \tilde{\tau})=\widetilde{\gamma(a, \tau}\right)$. Note that for any $\phi, \psi \in \mathfrak{g}^{*}$ and $g \in G$, there exist $a \in C^{\infty}(G), s \in C^{\infty}\left(G, \mathfrak{g}^{*}\right)$ such that $\hat{L}_{a}(g)=\phi$ and $s(g)=\psi$. One can define a map $\widetilde{\Xi}: G \times \mathfrak{g}^{*} \times \mathfrak{g}^{*} \rightarrow \mathfrak{g}^{*}$ by

$$
\tilde{\gamma}(a, s)(g)=\{a, s\}(g)+\widetilde{\Xi}\left(g, \hat{L}_{a}(g), s(g)\right) .
$$

For brevity, the notation for the Poisson bracket is extended to include $\mathfrak{g}^{*}$-valued functions on one side.

Beggs and Majid [2006, Proposition 4.5] show that a preconnection $\gamma$ is leftcovariant if and only if $\widetilde{\Xi}(g h, \phi, \psi)=\widetilde{\Xi}(h, \phi, \psi)$ for any $g, h \in G$ and $\phi, \psi \in \mathfrak{g}^{*}$. Hence for a left-covariant preconnection the map $\widetilde{\Xi}$ defines a map $\Xi: \mathfrak{g}^{*} \otimes \mathfrak{g}^{*} \rightarrow \mathfrak{g}^{*}$ by $\Xi(\phi, \psi)=\widetilde{\Xi}(e, \phi, \psi)$ and conversely, given $\Xi: \mathfrak{g}^{*} \otimes \mathfrak{g}^{*} \rightarrow \mathfrak{g}^{*}$,

$$
\tilde{\gamma}(a, s)(g)=\{a, s\}(g)+\Xi\left(\hat{L}_{a}(g), s(g)\right)
$$

defines the corresponding left-covariant preconnection $\gamma$. In addition, Beggs and Majid [2006, Proposition 4.6] show that a left-covariant preconnection is Poissoncompatible if and only if the corresponding $\Xi$ obeys

$$
\Xi(\phi, \psi)-\Xi(\psi, \phi)=[\phi, \psi]_{\mathfrak{g}^{*}}
$$

for all $\phi, \psi \in \mathfrak{g}^{*}$.

Based on these results, we can write down a formula for the preconnection $\gamma$ in coordinates. Let $\left\{e_{i}\right\}$ be a basis of $\mathfrak{g}$ and $\left\{f^{i}\right\}$ be the dual basis of $\mathfrak{g}^{*}$. Let $\left\{\omega^{i}\right\}$ be the basis of left-invariant 1 -forms that is dual to $\left\{\partial_{i}\right\}$ the left-invariant vector fields (generated by $\left\{e_{i}\right\}$ ) of $G$. Then the Maurer-Cartan form is

$$
\omega=\sum_{i} \omega^{i} e_{i} \in \Omega^{1}(G, \mathfrak{g}) .
$$

For any $\eta=\sum_{i} \eta_{i} \omega^{i} \in \Omega^{1}(G)$ with $\eta_{i} \in C^{\infty}(G)$, we know $\eta$ corresponds to $\tilde{\eta}=\sum_{i} \eta_{i} f^{i} \in C^{\infty}\left(G, \mathfrak{g}^{*}\right)$. On the other hand, any $s=\sum_{i} s_{i} f^{i} \in C^{\infty}\left(G, \mathfrak{g}^{*}\right)$ with $s_{i} \in C^{\infty}(G)$ corresponds to $\hat{s}=\sum_{i} s_{i} \omega^{i} \in \Omega^{1}(G)$. In particular, $\tilde{\mathrm{d} a}=\sum_{i}\left(\partial_{i} a\right) f^{i}$.

For any $a \in C^{\infty}(G)$ and $\tau=\sum_{i} \tau_{i} \omega^{i} \in \Omega^{1}(G)$, we know $\{a, \tilde{\tau}\}=\sum_{i}\left\{a, \tau_{i}\right\} f^{i}$ and

$$
\begin{aligned}
\Xi(\tilde{\mathrm{d} a}(g), \tilde{\tau}(g)) & =\Xi\left(\sum_{i}\left(\partial_{i} a\right)(g) f^{i}, \sum_{j} \tau_{j}(g) f^{j}\right) \\
& =\sum_{i, j}\left(\partial_{i} a\right)(g) \tau_{j}(g) \Xi\left(f^{i}, f^{j}\right) \\
& =\sum_{i, j, k}\left(\partial_{i} a\right)(g) \tau_{j}(g)\left\langle\Xi\left(f^{i}, f^{j}\right), e_{k}\right\rangle f^{k},
\end{aligned}
$$


so

$$
\tilde{\gamma}(a, \tilde{\tau})=\sum_{k}\left(\left\{a, \tau_{k}\right\}+\sum_{i, j}\left(\partial_{i} a\right) \tau_{j}\left\langle\Xi\left(f^{i}, f^{j}\right), e_{k}\right\rangle\right) f^{k}
$$

If we write $\Xi_{k}^{i j}=\left\langle\Xi\left(f^{i}, f^{j}\right), e_{k}\right\rangle\left(\right.$ or $\left.\Xi\left(f^{i}, f^{j}\right)=\sum_{k} \Xi_{k}^{i j} f^{k}\right)$ for any $i, j, k$, then we have

$$
\gamma(a, \tau)=\sum_{k}\left(\left\{a, \tau_{k}\right\}+\sum_{i, j} \Xi_{k}^{i j}\left(\partial_{i} a\right) \tau_{j}\right) \omega^{k}
$$

In particular, we have a more handy formula,

$$
\gamma\left(a, \omega^{j}\right)=\sum_{i, k}\left(\partial_{i} a\right)\left\langle\Xi\left(f^{i}, f^{j}\right), e_{k}\right\rangle \omega^{k}=\sum_{i, k} \Xi_{k}^{i j}\left(\partial_{i} a\right) \omega^{k} \quad \text { for all } j
$$

\section{Bicovariant preconnections}

Beggs and Majid [2006, Theorem 4.14] show that $\gamma$ is bicovariant at the Poisson-Lie group level if and only if

$$
\Xi(\phi, \psi)-\operatorname{Ad}_{g^{-1}}^{*} \Xi\left(\operatorname{Ad}_{g}^{*} \phi, \operatorname{Ad}_{g}^{*} \psi\right)=\phi\left(g^{-1} \pi^{(1)}(g)\right) \operatorname{ad}_{g^{-1} \pi^{(2)}(g)}^{*} \psi
$$

for all $g \in G$ and $\phi, \psi \in \mathfrak{g}^{*}$. We now give a new characterisation in terms of Lie bialgebra-level data.

Theorem 3.1. Let $G$ be a connected and simply connected Poisson-Lie group. A left-covariant preconnection on $G$ determined by $\Xi: \mathfrak{g}^{*} \otimes \mathfrak{g}^{*} \rightarrow \mathfrak{g}^{*}$ is bicovariant if and only if $\left(\mathrm{ad}^{*},-\Xi\right)$ makes $\mathfrak{g}^{*}$ into a left almost $\mathfrak{g}$-crossed module, or explicitly,

$$
\operatorname{ad}_{x}^{*} \Xi(\phi, \psi)-\Xi\left(\operatorname{ad}_{x}^{*} \phi, \psi\right)-\Xi\left(\phi, \operatorname{ad}_{x}^{*} \psi\right)=\phi\left(x_{(1)}\right) \operatorname{ad}_{x_{(2)}}^{*}(\psi)
$$

for all $x \in \mathfrak{g}$ and $\phi, \psi \in \mathfrak{g}^{*}$, where $\delta(x)=x_{(1)} \otimes x_{(2)}$. This is equivalent to

$$
\delta_{\mathfrak{g}^{*}} \Xi(\phi, \psi)-\Xi\left(\phi_{(1)}, \psi\right) \otimes \phi_{(2)}-\Xi\left(\phi, \psi_{(1)}\right) \otimes \psi_{(2)}=\psi(1) \otimes\left[\phi, \psi_{(2)}\right]_{\mathfrak{g}^{*}}
$$

for all $\phi, \psi \in \mathfrak{g}^{*}$.

Proof. We first show the "only if" part. To obtain the corresponding formula at the Lie algebra level for (3-1), we substitute $g$ with $\exp t x$ and differentiate at $t=0$. Notice that $\mathrm{d} \mathrm{Ad}^{*}(\exp t x) /\left.\mathrm{d} t\right|_{t=0}=\operatorname{ad}_{x}^{*}$ and $\left.\mathrm{Ad}^{*}(\exp t x)\right|_{t=0}=\mathrm{id}_{\mathfrak{g}^{*}}$. This gives (3-2) as stated, where $\delta(x)=x_{(1)} \otimes x_{(2)}=\mathrm{d} g^{-1} P(g) /\left.\mathrm{d} t\right|_{t=0}$ when $g=\exp t x$. Now denote $\operatorname{ad}_{x}^{*}$ by $x \triangleright$ and let $-\Xi(\phi)=,\phi \triangleright$, the left $\mathfrak{g}^{* \text { op }}$-action; then the left-hand side of (3-2) becomes

$$
-x \triangleright(\phi \triangleright \psi)+\phi_{(1)} \triangleright \psi\left\langle\phi_{(2)}, x\right\rangle+\phi \triangleright(x \triangleright \psi),
$$


while the right-hand side is

$$
\phi\left(x_{(1)}\right) \operatorname{ad}_{x_{(2)}}^{*}(\psi)=-\phi\left(x_{(2)}\right) \operatorname{ad}_{x_{(1)}}^{*}(\psi)=-x_{(1)} \triangleright \psi\left\langle\phi, x_{(2)}\right\rangle .
$$

Hence (3-2) is the content of

$$
\phi_{(1)} \triangleright \psi\left\langle\phi_{(2)}, x\right\rangle+x_{(1)} \triangleright \psi\left\langle\phi, x_{(2)}\right\rangle=x \triangleright(\phi \triangleright \psi)-\phi \triangleright(x \triangleright \psi)
$$

in our case, i.e., that $\mathfrak{g}^{*}$ is a left almost $\mathfrak{g}$-crossed module under $\left(\mathrm{ad}^{*},-\Xi\right)$.

Conversely, we can exponentiate $x$ near zero, and solve the ordinary differential equation (3-2) near $g=e$. It has a unique solution (3-1) near the identity. Since the Lie group $G$ is connected and simply connected, one can show that (3-1) is valid on the whole group.

Notice that $\operatorname{ad}_{x}^{*} \phi=\phi_{(1)}\left\langle\phi_{(2)}, x\right\rangle$ for any $x \in \mathfrak{g}$ and $\phi \in \mathfrak{g}^{*}$, so the left-hand side of (3-2) becomes

$$
-\Xi(\phi, \psi)_{(1)}\left\langle\Xi(\phi, \psi)_{(2)}, x\right\rangle-\Xi\left(\phi_{(1)}, \psi\right)\left\langle\phi_{(2)}, x\right\rangle-\Xi\left(\phi, \psi_{(1)}\right)\left\langle\psi_{(2)}, x\right\rangle,
$$

while the right-hand side of (3-2) is

$$
\phi\left(x_{(1)}\right) \psi_{(1)}\left\langle\psi_{(2)}, x_{(2)}\right\rangle=\psi_{(1)}\left\langle\left[\phi, \psi_{(2)}\right]_{\mathfrak{g}^{*}}, x\right\rangle,
$$

thus (3-2) is equivalent to (3-3) by using the duality pairing between $\mathfrak{g}$ and $\mathfrak{g}^{*}$.

\section{Flat preconnections}

As in [Beggs and Majid 2006], the curvature of a preconnection $\gamma$ is defined on Hamiltonian vector fields $\hat{x}=\{x,-\}$ by

$$
R(x, y) \tau=\gamma(x, \gamma(y, \tau))-\gamma(y, \gamma(x, \tau))-\gamma(\{x, y\}, \tau) \text { for all } \tau \in \Omega^{1}(G),
$$

which agrees with the covariant derivative curvature along Hamiltonian vector fields $\hat{x}, \hat{y}$ when this applies, on noting that $[\hat{x}, \hat{y}]=\{\widehat{x, y}\}$. The curvature of a preconnection reflects the obstruction to the Jacobi identity on any functions $x, y$ and 1-form $\tau$ up to third order, namely

$$
\left[x,[y, \tau]_{\bullet}\right]_{\bullet}+\left[y,[\tau, x]_{\bullet}\right]_{\bullet}+\left[\tau,[x, y]_{\bullet}\right]_{\bullet}=\lambda^{2} R(\hat{x}, \hat{y})(\tau)+O\left(\lambda^{3}\right) .
$$

This is the deformation-theoretic meaning of curvature in this context. We say a preconnection is flat if its curvature is zero. This takes a similar form in terms of $\tilde{\gamma}$, namely

$$
\tilde{\gamma}(x, \tilde{\gamma}(y, s))-\tilde{\gamma}(y, \tilde{\gamma}(x, s))-\tilde{\gamma}(\{x, y\}, s)=0
$$

for all $x, y \in C^{\infty}(G)$ and $s \in C^{\infty}\left(G, \mathfrak{g}^{*}\right)$.

Theorem 4.1. Let $G$ be a connected and simply connected Poisson-Lie group with Lie algebra $\mathfrak{g}$ and $\gamma$ a Poisson-compatible left-covariant preconnection. 
(i) $\gamma$ is flat if and only if the corresponding map $-\Xi$ is a right $\mathfrak{g}^{*}$-action (or left $\mathfrak{g}^{* 0 \mathrm{p}}$-action) on $\mathfrak{g}^{*}$,

(4-2) $\Xi\left([\phi, \psi]_{\mathfrak{g}^{*}}, \zeta\right)=\Xi(\phi, \Xi(\psi, \zeta))-\Xi(\psi, \Xi(\phi, \zeta))$ for all $\phi, \psi, \zeta \in \mathfrak{g}^{*}$.

(ii) $\gamma$ is bicovariant and flat if and only if $\left(\mathrm{ad}^{*},-\Xi\right)$ makes $\mathfrak{g}^{*}$ a left $\mathfrak{g}$-crossed module.

Proof. Let $\gamma$ be a Poisson-compatible left-covariant preconnection on a Poisson-Lie group $G$. Firstly, we can rewrite formula (4-1) in terms of $\Xi: \mathfrak{g}^{*} \otimes \mathfrak{g}^{*} \rightarrow \mathfrak{g}^{*}$. By definition, the three terms in (4-1) become

$$
\begin{aligned}
\tilde{\gamma}(x, \tilde{\gamma}(y, s))(g)=\{x,\{y, s\}\} & (g)+\left\{x, \Xi\left(\hat{L}_{y}(g), s(g)\right)\right\} \\
& +\Xi\left(\hat{L}_{x}(g),\{y, s\}(g)\right)+\Xi\left(\hat{L}_{x}(g), \Xi\left(\hat{L}_{y}(g), s(g)\right)\right),
\end{aligned}
$$$$
\tilde{\gamma}(y, \tilde{\gamma}(x, s))(g)=\{y,\{x, s\}\}(g)+\left\{y, \Xi\left(\hat{L}_{x}(g), s(g)\right)\right\}
$$$$
+\Xi\left(\hat{L}_{y}(g),\{x, s\}(g)\right)+\Xi\left(\hat{L}_{y}(g), \Xi\left(\hat{L}_{x}(g), s(g)\right)\right),
$$

and

$$
\tilde{\gamma}(\{x, y\}, s)(g)=\{\{x, y\}, s\}(g)+\Xi\left(\hat{L}_{\{x, y\}}(g), s(g)\right) .
$$

Cancelling terms involving the Jacobi identity of a Poisson bracket, formula (4-1) becomes

$$
\begin{array}{r}
\left\{x, \Xi\left(\hat{L}_{y}(g), s(g)\right)\right\}+\Xi\left(\hat{L}_{x}(g),\{y, s\}(g)\right)+\Xi\left(\hat{L}_{x}(g), \Xi\left(\hat{L}_{y}(g), s(g)\right)\right) \\
-\left\{y, \Xi\left(\hat{L}_{x}(g), s(g)\right)\right\}-\Xi\left(\hat{L}_{y}(g),\{x, s\}(g)\right)-\Xi\left(\hat{L}_{y}(g), \Xi\left(\hat{L}_{x}(g), s(g)\right)\right) \\
=\Xi\left(\hat{L}_{\{x, y\}}(g), s(g)\right) .
\end{array}
$$

Note that since $\gamma$ is Poisson-compatible, this implies

$$
\begin{aligned}
\hat{L}_{\{x, y\}}(g) & =\tilde{\gamma}\left(x, \hat{L}_{y}\right)(g)-\tilde{\gamma}\left(y, \hat{L}_{x}\right)(g) \\
& =\left\{x, \hat{L}_{y}\right\}(g)+\Xi\left(\hat{L}_{x}(g), \hat{L}_{y}(g)\right)-\left\{y, \hat{L}_{x}\right\}(g)-\Xi\left(\hat{L}_{y}(g), \hat{L}_{x}(g)\right) .
\end{aligned}
$$

and $\left\{x, \Xi\left(\hat{L}_{y}(g), s(g)\right\}=\Xi\left(\left\{x, \hat{L}_{y}\right\}(g), s(g)\right)+\Xi\left(\hat{L}_{y}(g),\{x, s\}(g)\right)\right.$ by the derivation property of $\{x,-\}$. In this case (4-1) is equivalent to

$$
\begin{aligned}
\Xi\left(\hat{L}_{x}(g), \Xi\left(\hat{L}_{y}(g), s(g)\right)\right)-\Xi\left(\hat{L}_{y}(g), \Xi\left(\hat{L}_{x}(g), s(g)\right)\right) \\
=\Xi\left(\Xi\left(\hat{L}_{x}(g), \hat{L}_{y}(g)\right)-\Xi\left(\hat{L}_{y}(g), \hat{L}_{x}(g)\right), s(g)\right)
\end{aligned}
$$

for all $x, y \in C^{\infty}(G)$ and $s \in C^{\infty}\left(G, \mathfrak{g}^{*}\right)$.

Now if $\gamma$ is flat, we can evaluate this equation at the identity $e$ of $G$, and for any $\phi, \psi, \zeta \in \mathfrak{g}^{*}$, set $\phi=\hat{L}_{x}(e), \psi=\hat{L}_{y}(e)$ and $\zeta=s(e)$ for some $x, y \in C^{\infty}(G)$ and $s \in C^{\infty}\left(G, \mathfrak{g}^{*}\right)$. Then (4-3) becomes

$$
\Xi(\Xi(\phi, \psi)-\Xi(\psi, \phi), \zeta)=\Xi(\phi, \Xi(\psi, \zeta))-\Xi(\psi, \Xi(\phi, \zeta)) .
$$


Using compatibility again, we get (4-2) as displayed. This also shows $\Xi$ is a left $\mathfrak{g}^{*}$-action on itself, or $\mathfrak{g}^{*}$ is a left $\mathfrak{g}^{* \text { op }}$-module via $-\Xi$.

Conversely, if $\mathfrak{g}^{*}$ is a left $\mathfrak{g}^{* 0 \mathrm{op}}$-module via $\triangleright: \mathfrak{g}^{*} \otimes \mathfrak{g}^{*} \rightarrow \mathfrak{g}^{*}$ and such that $-\phi \triangleright \psi+\psi \triangleright \phi=[\phi, \psi]_{\mathfrak{g}^{*}}$, i.e., (4-2) holds. This implies (4-3) for any $x, y \in C^{\infty}(G)$, $s \in C^{\infty}\left(G, \mathfrak{g}^{*}\right)$, which is equivalent to (4-1).

The second part of the theorem combines the first part with Theorem 3.1.

4A. Preconnections and pre-Lie algebras. Now we recall the notion of a left preLie algebra (also known as a Vinberg algebra or left symmetric algebra). An algebra $(A, \circ)$, not necessarily associative, with product $\circ: A \otimes A \rightarrow A$ is called a (left) pre-Lie algebra if the identity

$$
(x \circ y) \circ z-(y \circ x) \circ z=x \circ(y \circ z)-y \circ(x \circ z)
$$

holds for all $x, y, z \in A$. From the definition, every associative algebra is a pre-Lie algebra and meanwhile every pre-Lie algebra $(A, \circ)$ admits a Lie algebra structure (denoted by $\mathfrak{g}_{A}$ ) with Lie bracket given by

$$
[x, y]_{\mathfrak{g}_{A}}:=x \circ y-y \circ x
$$

for all $x, y \in A$. The Jacobi identity of [ , $]_{\mathfrak{g}_{A}}$ holds automatically due to (4-4). With this in mind, we can rephrase Theorems 3.1 and 4.1 as follows.

Corollary 4.2. A connected and simply connected Poisson-Lie group G with Lie algebra $\mathfrak{g}$ admits a Poisson-compatible left-covariant flat preconnection if and only if $\left(\mathfrak{g}^{*},[,]_{\mathfrak{g}^{*}}\right)$ admits a pre-Lie structure $\Xi$. Moreover, this left-covariant preconnection is bicovariant if and only if $\Xi$ in addition obeys

$$
\begin{aligned}
\delta_{\mathfrak{g}^{*}} \Xi(\phi, \psi)-\Xi\left(\phi, \psi_{(1)}\right) \otimes \psi_{(2)}-\psi_{(1)} \otimes \Xi\left(\phi, \psi_{(2)}\right) & \\
& =\Xi\left(\phi_{(1)}, \psi\right) \otimes \phi_{(2)}-\psi_{(1)} \otimes \Xi\left(\psi_{(2)}, \phi\right)
\end{aligned}
$$

for all $\phi, \psi \in \mathfrak{g}^{*}$.

Proof. The first part is shown by (2-6) and (4-2). For the bicovariant case, the additional condition on $\Xi$ is (3-3). Using compatibility and rearranging terms, we know that (3-3) is equivalent to (4-6) as displayed.

Example 4.3. Let $\mathfrak{m}$ be a finite-dimensional Lie algebra and $G=\mathfrak{m}^{*}$ be an abelian Poisson-Lie group with its Kirillov-Kostant Poisson-Lie group structure $\{x, y\}=$ $[x, y]$ for all $x, y \in \mathfrak{m} \subset C^{\infty}\left(\mathfrak{m}^{*}\right)$ or $S(\mathfrak{m})$ in an algebraic context. By Corollary 4.2, this admits a Poisson-compatible left-covariant flat preconnection if and only if $\mathfrak{m}$ admits a pre-Lie algebra structure $\circ$. This preconnection is always bicovariant as (4-6) vanishes when Lie algebra $\mathfrak{m}^{*}$ is abelian $\left(\delta_{\mathfrak{m}}=0\right)$. Then (2-7) with $\Xi=0$ implies

$$
\gamma(x, \mathrm{~d} y)=\mathrm{d}(x \circ y) \quad \text { for all } x, y \in \mathfrak{m} .
$$


(Note that $\widetilde{\mathrm{d} y}$ is a constant-valued function in $C^{\infty}(G, \mathfrak{m})$, so $\{x, \widetilde{\mathrm{d} y}\} \equiv 0$ and $\tilde{\gamma}(x, \tilde{\mathrm{d} y})=\boldsymbol{\Xi}(x, y)$.)

In fact the algebra and its calculus in this example work to all orders. Thus the quantisation of $C^{\infty}\left(\mathfrak{m}^{*}\right)$ is $U_{\lambda}(\mathfrak{m})$, defined as a version of the enveloping algebra with relations $x y-y x=\lambda[x, y]$ for all $x, y \in \mathfrak{m}$, where we introduce a deformation parameter. If $\mathfrak{m}$ has an underlying pre-Lie structure then the above results lead to relations

$$
[x, \mathrm{~d} y]=\lambda \mathrm{d}(x \circ y) \text { for all } x, y \in \mathfrak{m},
$$

and one can check that this works exactly and not only to order $\lambda$ precisely as a consequence of the pre-Lie algebra axiom. The full result here is:

Proposition 4.4. Let $\mathfrak{m}$ be a finite-dimensional Lie algebra over a field $k$ of characteristic zero. Then connected bicovariant calculi $\Omega^{1}$ of classical dimension (i.e., $\left.\operatorname{dim} \Lambda^{1}=\operatorname{dim} \mathfrak{m}\right)$ on the enveloping algebra $U(\mathfrak{m})$ are in one-to-one correspondence with pre-Lie structures on $\mathfrak{m}$.

Proof. A differential calculus is said to be connected if $k e r \mathrm{~d}=k 1$ (as for a connected manifold classically). It is clear from [Majid and Tao 2015b, Propositions 2.11 and 4.7] that a bicovariant differential graded algebra on $U(\mathfrak{m})$ with left-invariant 1 -forms $\mathfrak{m}$ as a vector space corresponds to a 1 -cocycle $Z_{\triangleleft}^{1}(\mathfrak{m}, \mathfrak{m})$ that extends to a surjective right $\mathfrak{m}$-module map $\omega: U(\mathfrak{m})^{+} \rightarrow \mathfrak{m}$. Here the derivation

$$
\mathrm{d}: U(\mathfrak{m}) \rightarrow \Omega^{1}(U(\mathfrak{m}))=U(\mathfrak{m}) \otimes \mathfrak{m}
$$

is given by $\mathrm{d} a=a_{(1)} \otimes \omega\left(\pi\left(a_{(2)}\right)\right)$ for any $a \in U(\mathfrak{m})$. Suppose that $\omega$ is such a map; we take $\zeta=\left.\omega\right|_{\mathfrak{m}} \in Z_{\triangleleft}^{1}(\mathfrak{m}, \mathfrak{m})$. For any $x \in \mathfrak{m}$ such that $\zeta(x)=0$, we have $\mathrm{d} x=1 \otimes \omega(x)=0$, then ker $\mathrm{d}=k 1$ implies $x=0$, so $\zeta$ is an injection, hence a bijection as $\mathfrak{m}$ is finite-dimensional. Now we can define a product $0: \mathfrak{m} \otimes \mathfrak{m} \rightarrow \mathfrak{m}$ by $x \circ y=-\zeta^{-1}(\zeta(y) \triangleleft x)$. The 1-cocycle property $\zeta([x, y])=\zeta(x) \triangleleft y-\zeta(y) \triangleleft x$ implies $[x, y]=\zeta^{-1}(\zeta(x) \triangleleft y-\zeta(y) \triangleleft x)=-y \circ x+x \circ y$ for all $x, y \in \mathfrak{m}$. Hence this makes $\mathfrak{m}$ into a left pre-Lie algebra as

$$
\begin{aligned}
{[x, y] \circ z } & =-\zeta^{-1}(\zeta(z) \triangleleft[x, y]) \\
& =\zeta^{-1}((\zeta(z) \triangleleft y) \triangleleft x)-\zeta^{-1}((\zeta(z) \triangleleft x) \triangleleft y) \\
& =x \circ(y \circ z)-y \circ(x \circ z) .
\end{aligned}
$$

Conversely, if $\mathfrak{m}$ admits a left pre-Lie structure $\circ$, then $y \triangleleft x=-x \circ y$ makes $\mathfrak{m}$ into a right $\mathfrak{m}$-module and $\zeta=\mathrm{id}_{\mathfrak{m}}$, the identity map, becomes a bijective 1 cocycle in $Z_{\triangleleft}^{1}(\mathfrak{m}, \mathfrak{m})$. The extended map $\omega: U(\mathfrak{m})^{+} \rightarrow \mathfrak{m}$ and the derivation $\mathrm{d}: U(\mathfrak{m}) \rightarrow U(\mathfrak{m}) \otimes \mathfrak{m}$ are given by $\omega\left(x_{1} x_{2} \cdots x_{n}\right)=\left(\left(x_{1} \triangleleft x_{2}\right) \triangleleft \cdots \triangleleft x_{n}\right)$ for any 
$x_{1} x_{2} \cdots x_{n} \in U(\mathfrak{m})^{+}$and

$$
\mathrm{d}\left(x_{1} x_{2} \cdots x_{n}\right)=\sum_{p=0}^{n-1} \sum_{\sigma \in \operatorname{Sh}(p, n-p)} x_{\sigma(1)} \cdots x_{\sigma(p)} \otimes \omega\left(x_{\sigma(p+1)} \cdots x_{\sigma(n)}\right)
$$

for any $x_{1} x_{2} \cdots x_{n} \in U(\mathfrak{m})$, respectively. We need to show that ker $\mathrm{d}=k 1$. On the one hand, $k 1 \subseteq$ kerd, as d(1) $=0$. On the other hand, denote by $U_{n}(\mathfrak{m})$ the subspace of $U(\mathfrak{m})$ generated by the products $x_{1} x_{2} \cdots x_{p}$, where $x_{1}, \ldots, x_{p} \in \mathfrak{m}$ and $p \leq n$. Clearly, $U_{0}=k 1, U_{1}(\mathfrak{m})=k 1 \oplus \mathfrak{m}, U_{p}(\mathfrak{m}) U_{q}(\mathfrak{m}) \subseteq U_{p+q}(\mathfrak{m})$ and thus $\left(U_{n}(\mathfrak{m})\right)_{n \geq 0}$ is a filtration of $U(\mathfrak{m})$. In order to show ker $d \subseteq k 1$, it suffices to show that the intersection

$$
(\text { kerd }) \cap U_{n}(\mathfrak{m})=k 1 \quad \text { for any integer } n \geq 0 .
$$

We prove this by induction on $n \geq 0$. It is obvious for $n=0$, and true for $n=1$ as, for any $v=\sum_{i} x_{i} \in($ ker $\mathrm{d}) \cap \mathfrak{m}, 0=\mathrm{d} v=\sum_{i} 1 \otimes \omega\left(x_{i}\right)=\sum_{i} 1 \otimes x_{i}$ implies $v=\sum_{i} x_{i}=0$. Suppose that (kerd) $\cap U_{n-1}(\mathfrak{m})=k 1$ for $n \geq 2$. For any $v \in$ $\left(\right.$ ker d) $\cap U_{n}(\mathfrak{m})$, without loss of generality we can write $v=\sum_{i} x_{i_{1}} x_{i_{2}} \cdots x_{i_{n}}+v^{\prime}$, where $x_{i_{j}} \in \mathfrak{m}$ and $v^{\prime}$ is an element in $U_{n-1}(\mathfrak{m})$. We have

$$
\begin{aligned}
\mathrm{d} v=\sum_{i} 1 \otimes \omega\left(x_{i_{1}} \cdots x_{i_{n}}\right)+\sum_{i} \sum_{j=1}^{n} x_{i_{1}} \cdots x_{i_{(j-1)}} \hat{x}_{i_{j}} x_{i_{(j+1)}} \cdots x_{i_{n}} \otimes x_{i_{j}} \\
+\sum_{i} \sum_{r=1}^{n-2} \sum_{\sigma \in \operatorname{Sh}(r, n-r)} x_{i_{\sigma(1)}} \cdots x_{i_{\sigma(r)}} \otimes \omega\left(x_{i_{\sigma(r+1)}} \cdots x_{i_{\sigma(n)}}\right)+\mathrm{d} v^{\prime} .
\end{aligned}
$$

We denote the elements

$$
u_{i_{j}}:=x_{i_{1}} \cdots x_{i_{(j-1)}} \hat{x}_{i_{j}} x_{i_{(j+1)}} \cdots x_{i_{n}} \in U_{n-1}(\mathfrak{m})
$$

for any $i, 1 \leq j \leq n$. Except the term $\sum_{i} \sum_{j=1}^{n} u_{i_{j}} \otimes x_{i_{j}}$, all the summands in $\mathrm{d} v$ lie in $U_{n-2}(\mathfrak{m}) \otimes \mathfrak{m}$, thus $\sum_{i} \sum_{j=1}^{n} u_{i_{j}} \otimes x_{i_{j}}$ also lies in $U_{n-2}(\mathfrak{m}) \otimes \mathfrak{m}$ as $\mathrm{d} v=0$. This implies $\sum_{i} \sum_{j=1}^{n} u_{i_{j}} x_{i_{j}}=v^{\prime \prime}$ for some element $v^{\prime \prime} \in U_{n-1}(\mathfrak{m})$. Rearrange this and add $n-1$ copies of $\sum_{i} u_{i_{n}} x_{i_{n}}=\sum_{i} x_{i_{1}} x_{i_{2}} \cdots x_{i_{n}}$ on both sides; we get

$$
n \sum_{i} x_{i_{1}} \cdots x_{i_{n}}=\sum_{i} \sum_{j=1}^{n-1} x_{i_{1}} \cdots x_{i_{(j-1)}}\left[x_{i_{j}}, x_{i_{j+1}} \cdots x_{i_{n}}\right]+v^{\prime \prime} ;
$$

therefore,

$$
v=\frac{1}{n} \sum_{i} \sum_{j=1}^{n-1} x_{i_{1}} \cdots x_{i_{(j-1)}}\left[x_{i_{j}}, x_{i_{j+1}} \cdots x_{i_{n}}\right]+\frac{1}{n} v^{\prime \prime}+v^{\prime} \in U_{n-1}(\mathfrak{m}) .
$$

Thus, we see that $v$ actually lies in (kerd) $\cap U_{n-1}(\mathfrak{m})$, hence $v \in k 1$ by assumption. Hence (kerd) $\cap U_{n}(\mathfrak{m})=k 1$ for any $n \geq 0$, which completes the proof. 
We apply this to $U_{\lambda}(\mathfrak{m})$. Because the Hopf algebra here is cocommutative, the canonical extension to a DGA is by the classical exterior or Grassmann algebra on $\Lambda^{1}=\mathfrak{m}$ with $\mathrm{d} \Lambda^{1}=0$. To make contact with real classical geometry in the rest of the paper, the standard approach in noncommutative geometry is to work over $\mathbb{C}$ with complexified differential forms and functions and to remember the "real form" by means of a $*$-involution. We recall that a differential graded algebra over $\mathbb{C}$ is called a $*$-DGA if it is equipped with a conjugate-linear map $*: \Omega \rightarrow \Omega$ such that

$$
*^{2}=\mathrm{id}, \quad(\xi \wedge \eta)^{*}=(-1)^{|\xi||\eta|} \eta^{*} \wedge \xi^{*}, \quad \mathrm{~d}\left(\xi^{*}\right)=(\mathrm{d} \xi)^{*}
$$

for any $\xi, \eta \in \Omega$. Let $\mathfrak{m}$ be a real pre-Lie algebra, i.e., there is a basis $\left\{e_{i}\right\}$ of $\mathfrak{m}$ with real structure coefficients. Then this is also a real form for $\mathfrak{m}$ as a Lie algebra. In this case, $e_{i}^{*}=e_{i}$ extends complex-linearly to an involution $*: \mathfrak{m} \rightarrow \mathfrak{m}$, which then makes $\Omega\left(U_{\lambda}(\mathfrak{m})\right)$ a $*$-DGA if $\lambda^{*}=-\lambda$, i.e., if $\lambda$ is imaginary. If we want $\lambda$ real then we should take $e_{i}^{*}=-e_{i}$.

Example 4.5. Let $\mathfrak{b}$ be the 2-dimensional complex nonabelian Lie algebra defined by $[x, t]=x$. It admits five families of mutually nonisomorphic pre-Lie algebra structures over $\mathbb{C}$ [Burde 1998], which are

$$
\begin{aligned}
& \mathfrak{b}_{1, \alpha}: \quad t \circ x=-x, \quad t \circ t=\alpha t, \\
& \mathfrak{b}_{2, \beta \neq 0}: \quad x \circ t=\beta x, \quad t \circ x=(\beta-1) x, \quad t \circ t=\beta t, \\
& \mathfrak{b}_{3}: \quad t \circ x=-x, \quad t \circ t=x-t \text {, } \\
& \mathfrak{b}_{4}: \quad x \circ x=t, \quad t \circ x=-x, \quad t \circ t=-2 t \text {, } \\
& \mathfrak{b}_{5}: \quad x \circ t=x, \quad t \circ t=x+t,
\end{aligned}
$$

where $\alpha, \beta \in \mathbb{C}$. (Here $\mathfrak{b}_{1,0} \cong \mathfrak{b}_{2,0}$, so we let $\beta \neq 0$.) Thus there are five families of bicovariant differential calculi over $U_{\lambda}(\mathfrak{b})$ :

$$
\begin{array}{llll}
\Omega^{1}\left(U_{\lambda}\left(\mathfrak{b}_{1, \alpha}\right)\right): & {[t, \mathrm{~d} x]=-\lambda \mathrm{d} x,} & {[t, \mathrm{~d} t]=\lambda \alpha \mathrm{d} t} \\
\Omega^{1}\left(U_{\lambda}\left(\mathfrak{b}_{2, \beta \neq 0}\right)\right): & {[x, \mathrm{~d} t]=\lambda \beta \mathrm{d} x,} & {[t, \mathrm{~d} x]=\lambda(\beta-1) \mathrm{d} x,} & {[t, \mathrm{~d} t]=\lambda \beta \mathrm{d} t} \\
\Omega^{1}\left(U_{\lambda}\left(\mathfrak{b}_{3}\right)\right): & {[t, \mathrm{~d} x]=-\lambda \mathrm{d} x,} & {[t, \mathrm{~d} t]=\lambda \mathrm{d} x-\lambda \mathrm{d} t ;} & \\
\Omega^{1}\left(U_{\lambda}\left(\mathfrak{b}_{4}\right)\right): & {[x, \mathrm{~d} x]=\lambda \mathrm{d} t,} & {[t, \mathrm{~d} x]=-\lambda \mathrm{d} x,} & {[t, \mathrm{~d} t]=-2 \lambda \mathrm{d} t} \\
\Omega^{1}\left(U_{\lambda}\left(\mathfrak{b}_{5}\right)\right): & {[x, \mathrm{~d} t]=\lambda \mathrm{d} x,} & {[t, \mathrm{~d} t]=\lambda \mathrm{d} x+\lambda \mathrm{d} t .} &
\end{array}
$$

All these examples are $*$-DGAs with $x^{*}=x$ and $t^{*}=t$ when $\lambda^{*}=-\lambda$ as $\{x, t\}$ is a real form of the relevant pre-Lie algebra. We also need for this that $\alpha$ and $\beta$ are real. The further noncommutative geometry of $\mathfrak{b}_{1, \alpha}$ and $\mathfrak{b}_{2, \beta}$ in 4-dimensional cases is studied in [Majid and Tao 2015a]. 
Example 4.6. For $q \in \mathbb{C}, q \neq 0$, we recall that the Hopf algebra $\mathbb{C}_{q}\left[\mathrm{SL}_{2}\right]$ is, as an algebra, a quotient of a free algebra $\mathbb{C}\langle a, b, c, d\rangle$ modulo relations

$$
\begin{gathered}
b a=q a b, \quad c a=q a c, \quad d b=q b d, \quad d c=q c d, \quad b c=c b, \\
a d-d a=\left(q^{-1}-q\right) b c, \quad a d-q^{-1} b c=1 .
\end{gathered}
$$

Writing the generators $a, b, c, d$ as a single matrix, the coproduct, counit and antipode of $\mathbb{C}_{q}\left[\mathrm{SL}_{2}\right]$ are given by

$$
\Delta\left(\begin{array}{ll}
a & b \\
c & d
\end{array}\right)=\left(\begin{array}{ll}
a & b \\
c & d
\end{array}\right) \otimes\left(\begin{array}{ll}
a & b \\
c & d
\end{array}\right), \quad \epsilon\left(\begin{array}{ll}
a & b \\
c & d
\end{array}\right)=\left(\begin{array}{ll}
1 & 0 \\
0 & 1
\end{array}\right), \quad S\left(\begin{array}{ll}
a & b \\
c & d
\end{array}\right)=\left(\begin{array}{cc}
d & -q b \\
-q^{-1} c & a
\end{array}\right),
$$

where we understand $\Delta(a)=a \otimes a+b \otimes c, \epsilon(a)=1, S(a)=d$, etc. By definition, the quantum group $\mathbb{C}_{q}\left[\mathrm{SU}_{2}\right]$ is Hopf algebra $\mathbb{C}_{q}\left[\mathrm{SL}_{2}\right]$ with $q$ real and $*$-structure

$$
\left(\begin{array}{ll}
a^{*} & b^{*} \\
c^{*} & d^{*}
\end{array}\right)=\left(\begin{array}{cc}
d & -q^{-1} c \\
-q b & a
\end{array}\right) \text {. }
$$

We use the conventions of [Majid 1995] and refer there for the history, which is related both to [Woronowicz 1989] and the Drinfeld theory [1987].

On $\mathbb{C}_{q}\left[\mathrm{SU}_{2}\right]$, there is a connected left-covariant calculus $\Omega^{1}\left(\mathbb{C}_{q}\left[\mathrm{SU}_{2}\right]\right)$ in [Woronowicz 1989] with basis, in our conventions,

$$
\omega^{0}=d \mathrm{~d} a-q b \mathrm{~d} c, \quad \omega^{+}=d \mathrm{~d} b-q b \mathrm{~d} d, \quad \omega^{-}=q a \mathrm{~d} c-c \mathrm{~d} a
$$

of left-invariant 1 -forms which is dual to the basis $\left\{\partial_{0}, \partial_{ \pm}\right\}$of left-invariant vector fields generated by the Chevalley basis $\left\{H, X_{ \pm}\right\}$of $\operatorname{su}_{2}$ (so that $\left[H, X_{ \pm}\right]= \pm 2 X_{ \pm}$ and $\left.\left[X_{+}, X_{-}\right]=H\right)$. The first-order calculus is generated by $\left\{\omega^{0}, \omega^{ \pm}\right\}$as a left module while the right module structure is given by the bimodule relations

$$
\omega^{0} f=q^{2|f|} f \omega^{0}, \quad \omega^{ \pm} f=q^{|f|} f \omega^{ \pm}
$$

for homogeneous $f$ of degree $|f|$, where $|a|=|c|=1,|b|=|d|=-1$, and with exterior derivatives

$$
\begin{array}{rlrl}
\mathrm{d} a & =a \omega^{0}+q^{-1} b \omega^{+}, & \mathrm{d} b=-q^{-2} b \omega^{0}+a \omega^{-}, \\
\mathrm{d} c=c \omega^{0}+q^{-1} d \omega^{+}, & \mathrm{d} d=-q^{-2} d \omega^{0}+c \omega^{-} .
\end{array}
$$

These extend to a differential graded algebra $\Omega\left(\mathbb{C}_{q}\left[\mathrm{SU}_{2}\right]\right)$ that has same dimension as classically. Moreover, it is a $*$-DGA with

$$
\omega^{0 *}=-\omega^{0}, \quad \omega^{+*}=-q^{-1} \omega^{-}, \quad \omega^{-*}=-q \omega^{+} .
$$

Since $\mathbb{C}_{q}\left[\mathrm{SU}_{2}\right]$ and $\Omega\left(\mathbb{C}_{q}\left[\mathrm{SU}_{2}\right]\right)$ are $q$-deformations, from Corollary 4.2 these must be quantised from some pre-Lie algebra structure of $s_{2}^{*}$, which we now compute. Let

$$
q=e^{\imath \lambda / 2}=1+\frac{l}{2} \lambda+O\left(\lambda^{2}\right)
$$


for imaginary $\lambda$. The Poisson bracket from the algebra relations is

$$
\begin{gathered}
\{a, b\}=-\frac{l}{2} a b, \quad\{a, c\}=-\frac{l}{2} a c, \quad\{a, d\}=-l b c, \quad\{b, c\}=0, \\
\{b, d\}=-\frac{l}{2} b d, \quad\{c, d\}=-\frac{l}{2} c d .
\end{gathered}
$$

The reader should not be alarmed by the $l$ as this is a "complexified" Poisson bracket on $C^{\infty}\left(\mathrm{SU}_{2}, \mathbb{C}\right)$ and is a real Poisson bracket on $C^{\infty}\left(\mathrm{SU}_{2}, \mathbb{R}\right)$ when we choose real-valued functions instead of complex-valued functions $a, b, c, d$ here.

As $\mathrm{d} x=\sum_{i}\left(\partial_{i} x\right) \omega^{i}$, we know, in the classical limit,

$$
\partial_{0}\left(\begin{array}{ll}
a & b \\
c & d
\end{array}\right)=\left(\begin{array}{ll}
a & -b \\
c & -d
\end{array}\right), \quad \partial_{+}\left(\begin{array}{ll}
a & b \\
c & d
\end{array}\right)=\left(\begin{array}{ll}
b & 0 \\
d & 0
\end{array}\right), \quad \partial_{-}\left(\begin{array}{ll}
a & b \\
c & d
\end{array}\right)=\left(\begin{array}{ll}
0 & a \\
0 & c
\end{array}\right) .
$$

From $a \omega^{0}-\omega^{0} a=\left(1-q^{2}\right) a \omega^{0}=-\imath \lambda a \omega^{0}+O\left(\lambda^{2}\right)$, we know that $\gamma\left(a, \omega^{0}\right)=$ $-\imath a \omega^{0}$. Likewise, we can get

$$
\gamma\left(\left(\begin{array}{ll}
a & b \\
c & d
\end{array}\right), \omega^{i}\right)=\frac{1}{2} t_{i}\left(\begin{array}{ll}
a & -b \\
c & -d
\end{array}\right) \omega^{i} \quad \text { for all } i \in\{0, \pm\}, t_{0}=-2 \imath, t_{ \pm}=-\imath .
$$

Now we can compute the pre-Lie structure $\Xi: \mathrm{su}_{2}^{*} \otimes \mathrm{su}_{2}^{*} \rightarrow \mathrm{su}_{2}^{*}$ by comparing with (2-8), namely

$$
\gamma\left(\left(\begin{array}{ll}
a & b \\
c & d
\end{array}\right), \omega^{j}\right)=\sum_{i, k \in\{0, \pm\}} \Xi_{k}^{i j}\left(\partial_{i}\left(\begin{array}{ll}
a & b \\
c & d
\end{array}\right)\right) \omega^{k}
$$

tells us that the only nonzero coefficients are

$$
\Xi_{0}^{00}=-l, \quad \Xi_{+}^{0+}=-\frac{l}{2}, \quad \Xi_{-}^{0-}=-\frac{l}{2} .
$$

Then

$$
\Xi(\phi, \phi)=-\imath \phi, \quad \Xi\left(\phi, \psi_{+}\right)=-\frac{l}{2} \psi_{+}, \quad \Xi\left(\phi, \psi_{-}\right)=-\frac{l}{2} \psi_{-},
$$

and $\Xi$ is zero on other terms, where $\left\{\phi, \psi_{ \pm}\right\}$is the dual basis of $\operatorname{su}_{2}^{*}$ to $\left\{H, X_{ \pm}\right\}$. Thus the corresponding pre-Lie structure of $\mathrm{su}_{2}^{*}$ is

$$
\Xi(\phi, \phi)=-\imath \phi, \quad \Xi\left(\phi, \psi_{ \pm}\right)=-\frac{l}{2} \psi_{ \pm} \quad \text { and zero otherwise. }
$$

Letting $t=-2 \imath \phi, x_{1}=\imath\left(\psi_{+}+\psi_{-}\right), x_{2}=\psi_{+}-\psi_{-}$, we have a real pre-Lie structure for $\mathrm{su}_{2}^{*}=\operatorname{span}\left\{t, x_{1}, x_{2}\right\}$ :

$$
t \circ t=-2 t, \quad t \circ x_{i}=-x_{i} \quad \text { for all } i=1,2 .
$$

This is a 3-dimensional version of $\mathfrak{b}_{1,-2}$.

Example 4.7. Let $\mathfrak{g}$ be a quasitriangular Lie bialgebra with $r$-matrix

$$
r=r^{(1)} \otimes r^{(2)} \in \mathfrak{g} \otimes \mathfrak{g} .
$$


Then $\mathfrak{g}$ acts on its dual $\mathfrak{g}^{*}$ by coadjoint action ad* and by [Majid 2000, Lemma 3.8], $\mathfrak{g}^{*}$ becomes a left $\mathfrak{g}$-crossed module with $-\Xi$, where $\Xi$ is the left $\mathfrak{g}^{*}$-action

$$
\Xi(\phi, \psi)=-\left\langle\phi, r^{(2)}\right\rangle \operatorname{ad}_{r^{(1)}}^{*} \psi .
$$

To satisfy the Poisson-compatibility $(2-6),(\mathfrak{g}, r)$ is required to obey

$$
r^{(1)} \otimes\left[r^{(2)}, x\right]+r^{(2)} \otimes\left[r^{(1)}, x\right]=0, \quad \text { i.e., } r_{+} \triangleright x=0, \quad \text { for all } x \in \mathfrak{g},
$$

where $r_{+}=\frac{1}{2}\left(r+r_{21}\right)$ is the symmetric part of $r$ and the second factor of $r_{+}$acts on $x$ via adjoint action of $\mathfrak{g}$. In this case $\mathfrak{g}^{*}$ has a pre-Lie algebra structure with $\Xi(\phi, \psi)=-\left\langle\phi, r^{(2)}\right\rangle \mathrm{ad}_{r^{(1)}}^{*} \psi$ by Corollary 4.2. We see in particular that every finite-dimensional cotriangular Lie bialgebra $\mathfrak{g}^{*}$ is canonically a pre-Lie algebra. More generally, if the centre $Z(\mathfrak{g})$ is nontrivial then any nonzero $r_{+} \in Z(\mathfrak{g})^{\otimes 2}$ combined with a triangular structure $r_{-}$gives a strictly quasitriangular $r=r_{-}+r_{+}$ obeying (4-7). This is the full content of (4-7) since this requires that the image of $r_{+}$regarded as a map $\mathfrak{g}^{*} \rightarrow \mathfrak{g}$ lies in $Z(\mathfrak{g})$ and $r_{+}$is symmetric. On the other hand, $\delta$ and $\Xi$ are the same as computed from $r_{-}$, so we may as well take $r_{+}=0$ as far as our present applications are concerned.

\section{Quantisation of the tangent bundle $T G=G \ltimes \underline{\mathfrak{g}}$}

We will be interested in quantisation of the tangent bundle $T G$ of a Poisson-Lie group $G$, with natural noncommutative coordinate algebra in this case provided by a bicrossproduct [Majid 1990b; 1995].

5A. Review of bicrossproduct Hopf algebras. We start with the notions of double cross-sum and bicross-sum of Lie bialgebras [Majid 1995, Chapter 8]. We say $(\mathfrak{g}, \mathfrak{m}, \triangleleft, \triangleright)$ forms a right-left matched pair of Lie algebras if $\mathfrak{g}$ and $\mathfrak{m}$ are both Lie algebras and $\mathfrak{g}$ right acts on $\mathfrak{m}$ via $\triangleleft, \mathfrak{m}$ left acts on $\mathfrak{g}$ via $\triangleright$ with

$$
\begin{aligned}
{[\phi, \psi] \triangleleft \xi } & =[\phi \triangleleft \xi, \psi]+[\phi, \psi \triangleleft \xi]+\phi \triangleleft(\psi \triangleright \xi)-\psi \triangleleft(\phi \triangleright \xi), \\
\phi \triangleright[\xi, \eta] & =[\phi \triangleright \xi, \eta]+[\xi, \phi \triangleright \eta]+(\phi \triangleleft \xi) \triangleright \eta-(\phi \triangleleft \eta) \triangleright \xi,
\end{aligned}
$$

for any $\xi, \eta \in \mathfrak{g}, \phi, \psi \in \mathfrak{m}$. Given such a matched pair, one can define the "double cross-sum Lie algebra" $\mathfrak{g} \bowtie \mathfrak{m}$ as the vector space $\mathfrak{g} \oplus \mathfrak{m}$ with the Lie bracket

$$
[(\xi, \phi),(\eta, \psi)]=([\xi, \eta]+\phi \triangleright \eta-\psi \triangleright \xi,[\phi, \psi]+\phi \triangleleft \eta-\psi \triangleleft \xi) .
$$

In addition, if both $\mathfrak{g}$ and $\mathfrak{m}$ are now Lie bialgebras with $\triangleright$ and $\triangleleft$ making $\mathfrak{g}$ a left $\mathfrak{m}$-module Lie coalgebra and $\mathfrak{m}$ a right $\mathfrak{g}$-module Lie coalgebra, such that

$$
\phi \triangleleft \xi_{(1)} \otimes \xi_{(2)}+\phi_{(1)} \otimes \phi_{(2)} \triangleright \xi=0
$$

for all $\xi \in \mathfrak{g}, \phi \in \mathfrak{m}$, then the direct sum Lie coalgebra structure makes $\mathfrak{g} \bowtie \mathfrak{m}$ into a Lie bialgebra, the double cross-sum Lie bialgebra. 
Next, if $\mathfrak{g}$ is finite-dimensional, the matched pair of Lie bialgebras $(\mathfrak{g}, \mathfrak{m}, \triangleleft, \triangleright)$ equivalently defines a right-left bicross-sum Lie bialgebra $\mathfrak{m} \bowtie \mathfrak{g}^{*}$ built on $\mathfrak{m} \oplus \mathfrak{g}^{*}$ with

$$
\begin{gathered}
{[(\phi, f),(\psi, h)]=\left([\phi, \psi]_{\mathfrak{m}},[f, h]_{\mathfrak{g}^{*}}+f \triangleleft \psi-h \triangleleft \phi\right),} \\
\delta \phi=\delta_{\mathfrak{m}} \phi+(\mathrm{id}-\tau) \beta(\phi), \quad \delta f=\delta_{\mathfrak{g}^{*}} f,
\end{gathered}
$$

for any $\phi, \psi \in \mathfrak{m}$ and $f, h \in \mathfrak{g}^{*}$, where the right action of $\mathfrak{m}$ on $\mathfrak{g}^{*}$ and the left coaction of $\mathfrak{g}^{*}$ on $\mathfrak{m}$ are induced from $\triangleleft$ and $\triangleright$ by

$$
\langle f \triangleleft \phi, \xi\rangle=\langle f, \phi \triangleright \xi\rangle, \quad \beta(\phi)=\sum_{i} f^{i} \otimes \phi \triangleleft e_{i},
$$

for all $\phi \in \mathfrak{m}, f \in \mathfrak{g}^{*}, \xi \in \mathfrak{g}$ and $\left\{e_{i}\right\}$ is a basis of $\mathfrak{g}$ with dual basis $\left\{f^{i}\right\}$. We refer to [Majid 1995, Section 8.3] for the proof.

Now let $(\mathfrak{g}, \mathfrak{m}, \triangleleft, \triangleright)$ be a matched pair of Lie algebras and $M$ be the connected and simply connected Lie group associated to $\mathfrak{m}$. The Poisson-Lie group $M \triangleright \mathfrak{g}^{*}$ associated to the bicross-sum $\mathfrak{m} \bowtie \mathfrak{g}^{*}$ is the semidirect product $M \bowtie \mathfrak{g}^{*}$ (where $\mathfrak{g}^{*}$ is regarded as an abelian group) equipped with Poisson bracket

$$
\{f, g\}=0, \quad\{\xi, \eta\}=[\xi, \eta]_{\mathfrak{g}}, \quad\{\xi, f\}=\alpha_{* \xi}(f),
$$

for all functions $f, g$ on $M$ and linear functions $\xi, \eta$ on $\mathfrak{g}^{*}$, where $\alpha_{* \xi}$ is the vector field for the action of $\mathfrak{g}$ on $M$. See [Majid 1995, Proposition 8.4.7] for the proof. Note that here $\mathfrak{g}, \mathfrak{m}$ are both viewed as Lie bialgebras with zero cobracket, so the Lie bracket and Lie cobracket of the bicross-sum Lie bialgebra $\mathfrak{m} \triangleright \mathfrak{g}^{*}$ is now given by (5-1) and (5-2) but with $[,]_{\mathfrak{g}^{*}}=0, \delta_{\mathfrak{m}}=0$.

More precisely, let $(\mathfrak{g}, \mathfrak{m}, \triangleleft, \triangleright)$ be a matched pair of Lie algebras, with the associated connected and simply connected Lie groups $G$ acting on $\mathfrak{m}$ and $M$ acting on $\mathfrak{g}$. The action $\triangleleft$ can be viewed as Lie algebra cocycle $\triangleleft \in Z_{\triangleright^{*} \otimes-\text { id }}^{1}\left(\mathfrak{m}, \mathfrak{g}^{*} \otimes \mathfrak{m}\right)$ and under some assumptions can then be exponentiated to a group cocycle

$$
a \in Z_{\triangleright^{*} \otimes \operatorname{Ad}_{R}}^{1}\left(M, \mathfrak{g}^{*} \otimes \mathfrak{m}\right),
$$

which defines an infinitesimal action of $\mathfrak{g}$ on $M$. Hence, by evaluation of the corresponding vector fields, $a$ defines a left action of the Lie algebra $\mathfrak{g}$ on $C^{\infty}(M)$ [Majid 1990a]:

$$
(\tilde{\xi} f)(s)=\tilde{a}_{\xi}(f)(s)=\left.\frac{\mathrm{d}}{\mathrm{d} t} f\left(s \exp \left(t a_{\xi}(s)\right)\right)\right|_{t=0} \quad \text { for all } f \in C^{\infty}(M), \xi \in \mathfrak{g} .
$$

We also note that $\mathfrak{m}$ acts on $M$ by a left-invariant vector field:

$$
(\widetilde{\phi} f)(s)=\left.\frac{\mathrm{d}}{\mathrm{d} t} f(s \exp (t \phi))\right|_{t=0}
$$


for any $\phi \in \mathfrak{m}, f \in C^{\infty}(M)$, and these two actions fit together to an action of $\mathfrak{g} \bowtie \mathfrak{m}$ on $C^{\infty}(M)$.

Finally, we can explain the bicrossproduct $\mathbb{C}[M] \bowtie U_{\lambda}(\mathfrak{g})$ based on a matched pair of Lie algebras $(\mathfrak{g}, \mathfrak{m}, \triangleleft, \triangleright)$, where $\mathbb{C}[M]$ is an algebraic model of functions on $M$. The algebra of $\mathbb{C}[M] \triangleleft U_{\lambda}(\mathfrak{g})$ is the cross product defined by the action (5-3). Its coalgebra, on the other hand, is the cross coproduct given in reasonable cases by a right coaction (defined by the left action of $M$ on $\mathfrak{g}$ )

$$
\beta: \mathfrak{g} \rightarrow \mathfrak{g} \otimes \mathbb{C}[M], \quad \beta(\xi)(s)=s \triangleright \xi \quad \text { for all } \xi \in \mathfrak{g}, s \in M .
$$

The map $\beta$ is extended to products of the generators of $U_{\lambda}(\mathfrak{g})$ to form a bicrossproduct $\mathbb{C}[M] \triangleleft U_{\lambda}(\mathfrak{g})$ as in [Majid 1995, Theorem 6.2.2].

The Poisson-Lie group $M \bowtie \mathfrak{g}^{*}$ quantises to $\mathbb{C}[M] \bowtie U_{\lambda}(\mathfrak{g})$ as a noncommutative deformation of the commutative algebra of functions $\mathbb{C}\left[M \triangleright \mathfrak{g}^{*}\right]$. See [Majid 1995, Section 8.3] for more details. The half-dualisation process we have described at the Lie bialgebra level also works at the Hopf algebra level, at least in the finitedimensional case. So morally speaking, $U_{\lambda}(\mathfrak{g}) \bowtie U(\mathfrak{m})$ half-dualises in a similar way to the bicrossproduct Hopf algebra $\mathbb{C}[M] \bowtie U_{\lambda}(\mathfrak{g})$. If one is only interested in the algebra and its calculus, we can extend to the cross product $C^{\infty}(M) \rtimes U_{\lambda}(\mathfrak{g})$.

5B. Poisson-Lie group structures on the tangent bundle $G \ltimes \mathfrak{g}$. Let $G$ be a Lie group with Lie algebra $\mathfrak{g}$. As a Lie group, the tangent bundle $T G$ of a Lie group $G$ can be identified with the semidirect product of Lie groups $G \ltimes \underline{\mathfrak{g}}$ (by the right adjoint action of $G$ on $\mathfrak{g}$ ) with product

$$
\left(g_{1}, x\right)\left(g_{2}, y\right)=\left(g_{1} g_{2}, \operatorname{Ad}\left(g_{2}^{-1}\right)(x)+y\right) \quad \text { for all } g_{1}, g_{2} \in G, x, y \in \mathfrak{g},
$$

where $\mathfrak{g}$ is $\mathfrak{g}$ but viewed as an abelian Poisson-Lie group under addition. Naturally, the Lie algebra of $G \ltimes \underline{\mathfrak{g}}$ is the semidirect sum Lie algebra $\mathfrak{g} \triangleright \underline{\mathfrak{g}}$ with Lie bracket

$$
[\xi, \eta]=[\xi, \eta]_{\mathfrak{g}}, \quad[x, y]=0, \quad[x, \xi]=[x, \xi]_{\mathfrak{g}} \quad \text { for all } \xi, \eta \in \mathfrak{g}, x, y \in \underline{\mathfrak{g}} .
$$

Keeping in mind the observations in Section 5A, we propose the following construction of a Poisson-Lie structure on the tangent bundle $G \ltimes \mathfrak{g}$ via a bicrosssum. In what follows we assume that $G$ is a finite-dimensional connected and simply connected Poisson-Lie group, and $\mathfrak{g}$ is its Lie algebra with the corresponding Lie bialgebra structure. We let

$$
\overline{\mathfrak{g}^{*}}:=\left(\mathfrak{g}^{*},[,]_{\mathfrak{g}^{*}} \text {, zero Lie cobracket }\right) \text { and } \overline{\mathfrak{g}}:=\left(\mathfrak{g},[,]_{\mathfrak{g}} \text {, zero Lie cobracket }\right),
$$

where $\overline{\mathfrak{g}^{*}}$ is the dual of Lie bialgebra $\underline{\mathfrak{g}}=\left(\mathfrak{g}\right.$, zero bracket, $\left.\delta_{\mathfrak{g}}\right)$. One can check that $\overline{\mathfrak{g}^{*}}$ and $\overline{\mathfrak{g}}$ together form a matched pair of Lie bialgebras with coadjoint actions, i.e.,

$$
\xi \triangleleft \phi=-\operatorname{ad}_{\phi}^{*} \xi=\left\langle\phi, \xi_{(1)}\right\rangle \xi_{(2)}, \quad \xi \triangleright \phi=\operatorname{ad}_{\xi}^{*} \phi=\phi_{(1)}\left\langle\phi_{(2)}, \xi\right\rangle
$$


for any $\phi \in \overline{\mathfrak{g}^{*}}, \xi \in \overline{\mathfrak{g}}$.

5B1. Lie bialgebra level. The double cross-sum Lie bialgebra $\overline{\mathfrak{g}^{*}} \bowtie \overline{\mathfrak{g}}$ is then built on $\mathfrak{g}^{*} \oplus \mathfrak{g}$ as a vector space with Lie bracket

$$
\begin{gathered}
{[\phi, \psi]=[\phi, \psi]_{\mathfrak{g}^{*}}, \quad[\xi, \eta]=[\xi, \eta]_{\mathfrak{g}},} \\
{[\xi, \phi]=\xi \triangleleft \phi+\xi \triangleright \phi=\left\langle\phi, \xi_{(1)}\right\rangle \xi_{(2)}+\phi_{(1)}\left\langle\phi_{(2)}, \xi\right\rangle}
\end{gathered}
$$

for all $\phi, \psi \in \overline{\mathfrak{g}^{*}}, \xi, \eta \in \overline{\mathfrak{g}}$, and zero Lie cobracket. This is nothing but the Lie algebra of the Drinfeld double $D(\mathfrak{g})=\mathfrak{g}^{*} \bowtie \mathfrak{g}$ of $\mathfrak{g}$ with zero Lie-cobracket.

Correspondingly, the right-left bicross-sum Lie bialgebra defined by the matched pair $\left(\overline{\mathfrak{g}^{*}}, \overline{\mathfrak{g}}, \triangleleft, \triangleright\right)$ above is $\overline{\mathfrak{g}} \triangleright \triangleleft \underline{\mathfrak{g}}$, whose Lie algebra is a semidirect sum $\overline{\mathfrak{g}} \bowtie \underline{\mathfrak{g}}$ and the Lie coalgebra is semidirect cobracket $\overline{\mathfrak{g}} \succ \underline{\mathfrak{g}}$, namely

$$
\begin{gathered}
{[\xi, \eta]=[\xi, \eta]_{\mathfrak{g}}, \quad[x, y]=0, \quad[x, \xi]=[x, \xi]_{\mathfrak{g}},} \\
\delta \xi=(\mathrm{id}-\tau) \delta_{\mathfrak{g}}(\xi)=\underline{\xi_{(1)}} \otimes \overline{\xi_{(2)}}-\overline{\xi_{(2)}} \otimes \underline{\xi_{(1)}}, \quad \delta x=\delta_{\mathfrak{g}} x,
\end{gathered}
$$

for any $\xi, \eta \in \overline{\mathfrak{g}}, x, y \in \underline{\mathfrak{g}}$. Here the coaction on $\overline{\mathfrak{g}}$ is the Lie cobracket $\delta_{\mathfrak{g}}$ viewed as a map from $\overline{\mathfrak{g}}$ to $\underline{\mathfrak{g}} \otimes \overline{\mathfrak{g}}$.

5B2. Poisson-Lie level. Associated to the right-left bicross-sum Lie bialgebra $\overline{\mathfrak{g}} \bowtie \underline{\mathfrak{g}}$, the Lie group $G \ltimes \underline{\mathfrak{g}}$ is a Poisson-Lie group (denoted by $\bar{G} \bowtie \underline{\mathfrak{g}}$ ) with the Poisson bracket

$$
\{f, h\}=0, \quad\{\phi, \psi\}=[\phi, \psi]_{\mathfrak{g}^{*}}, \quad\{\phi, f\}=\widetilde{\phi} f
$$

for any $\phi, \psi \in \overline{\mathfrak{g}^{*}} \subseteq C^{\infty}(\underline{\mathfrak{g}})$ and $f, h \in C^{\infty}(\bar{G})$, where $\widetilde{\phi}$ denotes the left Lie algebra action of $\overline{\mathfrak{g}^{*}}$ on $C^{\infty}(G)$ (viewed as a vector field on $G$ ) and is defined by the right action of $\overline{\mathfrak{g}^{*}}$ on $\overline{\mathfrak{g}}$.

The vector field $\widetilde{\phi}$ for any $\phi \in \mathfrak{g}^{*}$ in this case can be interpreted more precisely. We can view the actions between $\overline{\mathfrak{g}^{*}}$ and $\overline{\mathfrak{g}}$ as Lie algebra 1-cocycles, namely the right coadjoint action $\triangleleft=-$ ad $^{*}: \overline{\mathfrak{g}} \otimes \overline{\mathfrak{g}^{*}} \rightarrow \overline{\mathfrak{g}}$ (of $\overline{\mathfrak{g}^{*}}$ on $\overline{\mathfrak{g}}$ ) is viewed as a map $\overline{\mathfrak{g}} \rightarrow\left(\overline{\mathfrak{g}^{*}}\right)^{*} \otimes \overline{\mathfrak{g}}=(\underline{\mathfrak{g}})^{* *} \otimes \overline{\mathfrak{g}}=\underline{\mathfrak{g}} \otimes \overline{\mathfrak{g}}$. It maps $\xi$ to

$$
\sum_{i} e_{i} \otimes \xi \triangleleft f^{i}=\sum_{i} e_{i} \otimes\left\langle f^{i}, \xi_{(1)}\right\rangle \xi_{(2)}=\xi_{(1)} \otimes \xi_{(2)},
$$

which is nothing but the Lie cobracket $\delta_{\mathfrak{g}}$ of $\mathfrak{g}$. Likewise, the left coadjoint action of $\overline{\mathfrak{g}}$ on $\overline{\mathfrak{g}^{*}}$ is viewed as the Lie cobracket $\delta_{\mathfrak{g}^{*}}$ of $\mathfrak{g}^{*}$. We already know that the Lie 1-cocycle $\delta_{\mathfrak{g}} \in Z_{- \text {ad }}^{1}(\mathfrak{g}, \mathfrak{g} \otimes \mathfrak{g})$ exponentiates to a group cocycle

$$
D^{\vee} \in Z_{\operatorname{Ad}_{R}}^{1}(G, \mathfrak{g} \otimes \mathfrak{g}),
$$

thus

$$
\widetilde{\phi}_{g}:=\left(L_{g}\right)_{*}\left((\phi \otimes \mathrm{id}) D^{\vee}(g)\right) \in T_{g} G, \quad \text { for all } g \in G,
$$


defines the vector field on $G$ in (5-5).

According to [Majid 1995, Proposition 8.4.7], the Poisson bivector on the tangent bundle $T G=\bar{G} \triangleright \bullet \mathfrak{g}$ is

$$
\pi=\sum_{i}\left(\partial_{i} \otimes \tilde{f}^{i}-\tilde{f}^{i} \otimes \partial_{i}\right)+\sum_{i, j, k} d_{k}^{i j} f^{k} \partial_{i} \otimes \partial_{j},
$$

where $\left\{\partial_{i}\right\}$ is the basis of left-invariant vector fields generated by the basis $\left\{e_{i}\right\}$ of $\mathfrak{g}$ and $\left\{f^{i}\right\}$ is the dual basis of $\mathfrak{g}^{*}$. Here

$$
P_{K K}=\sum_{i, j, k} d_{k}^{i j} f^{k} \partial_{i} \otimes \partial_{j}
$$

is the known Kirillov-Kostant bracket on $\mathfrak{g}$ with

$$
\delta_{\mathfrak{g}} e_{k}=\sum_{i j} d_{k}^{i j} e_{i} \otimes e_{j}
$$

We arrive at the following special case of [Majid 1995, Proposition 8.4.7]:

Lemma 5.1. Let $G$ be a finite-dimensional connected and simply connected PoissonLie group and $\mathfrak{g}$ be its Lie algebra. The tangent bundle $T G=G \ltimes \underline{\mathfrak{g}}$ of $G$ admits a Poisson-Lie structure given by (5-5) or (5-7), denoted by $\bar{G} \bowtie \underline{\text { g. }}$. The corresponding Lie bialgebra is $\overline{\mathfrak{g}} \triangleright \mathbb{\mathfrak { g }}$, given by (5-4).

5B3. Bicrossproduct Hopf algebra. Finally, when the actions and coactions are suitably algebraic, we have a bicrossproduct Hopf algebra $\mathbb{C}[\bar{G}] \triangleleft U_{\lambda}\left(\overline{\mathfrak{g}^{*}}\right)$ as a quantisation of the commutative algebra of functions $\mathbb{C}[\bar{G} \triangleright \mathbf{g}]$ on the tangent bundle $\bar{G} \bowtie \mathfrak{g}$ of a Poisson-Lie group $G$. The commutation relations of $\mathbb{C}[\bar{G}] \bowtie U_{\lambda}\left(\overline{\mathfrak{g}^{*}}\right)$ are

$$
[f, h]=0, \quad[\phi, \psi]=\lambda[\phi, \psi]_{\mathfrak{g}^{*}}, \quad[\phi, f]=\lambda \widetilde{\phi} f
$$

for any $\phi, \psi \in \overline{\mathfrak{g}^{*}} \subseteq C^{\infty}(\mathfrak{g})$ and $f, h \in \mathbb{C}[\bar{G}]$. This construction is still quite general but includes a canonical example for all compact real forms $\mathfrak{g}$ of complex simple Lie algebras based in the Iwasawa decomposition to provide the double cross product or "Manin triple" in this case [Majid 1990a]. We start with an even simpler example.

Example 5.2. Let $\mathfrak{m}$ be a finite-dimensional real Lie algebra, viewed as a Lie bialgebra with zero Lie-cobracket. Take $G=\mathfrak{m}^{*}$, the abelian Poisson-Lie group with Kirillov-Kostant Poisson bracket given by the Lie bracket of $\mathfrak{m}$. Then $\mathfrak{g}=\mathfrak{m}^{*}$ and $\overline{\mathfrak{g}^{*}}=\mathfrak{m}$ and $\overline{\mathfrak{g}}=\overline{\mathfrak{m}^{*}}=\mathbb{R}^{n}$, where $n=\operatorname{dim} \mathfrak{m}$. Since the Lie bracket of $\mathfrak{m}^{*}$ is zero, $\overline{\mathfrak{m}^{*}}$ acts trivially on $\mathfrak{m}$, while $\mathfrak{m}$ acts on $\overline{\mathfrak{m}^{*}}$ by right coadjoint action $-\mathrm{ad}^{*}$, namely

$$
f \triangleleft \xi=-\operatorname{ad}_{\xi}^{*} f, \quad \text { or } \quad\langle f \triangleleft \xi, \eta\rangle=\left\langle f,[\xi, \eta]_{\mathfrak{m}}\right\rangle
$$

for any $f \in \overline{\mathfrak{m}^{*}}, \xi, \eta \in \mathfrak{m}$. So $\left(\mathfrak{m}, \overline{\mathfrak{m}^{*}}, \triangleleft=-\mathrm{ad}^{*}, \triangleright=0\right)$ forms a matched pair. 
The double cross-sum of the matched pair $\left(\mathfrak{m}, \overline{\mathfrak{m}^{*}}\right)$ is $\mathfrak{m} \bowtie \overline{\mathfrak{m}^{*}}$, the semidirect sum Lie algebra with coadjoint action of $\mathfrak{m}$ on $\overline{\mathfrak{m}^{*}}$ :

$$
\begin{gathered}
{[\xi, \eta]=[\xi, \eta]_{\mathfrak{m}}, \quad[f, h]=0, \quad[f, \xi]=f \triangleleft \xi=\left\langle\xi, f_{(1)}\right\rangle f_{(2)},} \\
\delta \xi=0, \quad \delta f=0 \quad \text { for all } \xi, \eta \in \mathfrak{m}, f, h \in \overline{\mathfrak{m}^{*}} .
\end{gathered}
$$

Meanwhile, the right-left bicross-sum of the matched pair $\left(\mathfrak{m}, \overline{\mathfrak{m}^{*}}\right)$ is $\overline{\mathfrak{m}^{*}} \backslash \mathfrak{m}^{*}$, the semidirect sum Lie coalgebra

$$
\begin{gathered}
{[f, h]=0, \quad[\phi, \psi]=0, \quad[\phi, f]=\phi \triangleleft f=0,} \\
\delta f=(\mathrm{id}-\tau) \beta(f), \quad \delta \phi=\delta_{\mathfrak{m}^{*}} \phi,
\end{gathered}
$$

for any $f, h \in \overline{\mathfrak{m}^{*}}, \phi, \psi \in \mathfrak{m}^{*}$, where the left coaction of $\mathfrak{m}^{*}$ on $\overline{\mathfrak{m}^{*}}$ is given by

$$
\beta: \overline{\mathfrak{m}^{*}} \rightarrow \mathfrak{m}^{*} \otimes \overline{\mathfrak{m}^{*}}, \quad \beta(f)=\sum_{i} f^{i} \otimes f \triangleleft e_{i},
$$

and $\left\{e_{i}\right\}$ is a basis of $\mathfrak{m}$ with dual basis $\left\{f^{i}\right\}$ of $\mathfrak{m}^{*}$.

The tangent bundle of $\mathfrak{m}^{*}$ is the associated Poisson-Lie group of $\overline{\mathfrak{m}^{*}}>\mathfrak{m}^{*}$, which is $\overline{M^{*}} \backslash \mathfrak{m}^{*}=\mathbb{R}^{n}>\mathfrak{m}^{*}$, an abelian Lie group, where we identify the abelian Lie group $\overline{M^{*}}$ with its abelian Lie algebra $\overline{\mathfrak{m}^{*}}$. Let $\left\{x^{i}\right\}$ be the coordinate functions on $\mathbb{R}^{n}$ identified with $\left\{e_{i}\right\} \subset \mathfrak{m} \subseteq C^{\infty}\left(\overline{\mathfrak{m}^{*}}\right)=C^{\infty}\left(\mathbb{R}^{n}\right)$, as $e_{i}\left(\sum_{j} \lambda_{j} f^{j}\right)=\lambda_{i}$. The right action of $\mathfrak{m}$ on $\overline{\mathfrak{m}^{*}}$ transfers to $\delta_{\mathfrak{m}^{*}} \in Z^{1}\left(\overline{\mathfrak{m}^{*}}, \underline{\mathfrak{m}^{*}} \otimes \overline{\mathfrak{m}^{*}}\right)$. As a Lie group $\overline{M^{*}}$ is abelian and $\overline{M^{*}}=\overline{\mathfrak{m}^{*}}=\mathbb{R}^{n}$, so the associated group cocycle is identical to $\delta_{\mathfrak{m}^{*}}$, thus from (5-6) we have

$$
\tilde{\xi}_{x} f=\left\langle x_{(1)}, \xi\right\rangle x_{(2) x} f=\sum_{i}\left\langle x_{(1)}, \xi\right\rangle\left\langle x_{(2)}, e_{i}\right\rangle f_{x}^{i} f=\sum_{i}\left\langle\left[\xi, e_{i}\right]_{\mathfrak{m}}, x\right\rangle \frac{\partial f}{\partial x^{i}}(x),
$$

where we use the Lie cobracket in an explicit notation. This shows that

$$
\tilde{\xi}=\sum_{i, j, k}\left\langle f^{i}, \xi\right\rangle c_{i j}^{k} x^{k} \frac{\partial}{\partial x^{j}} \quad \text { for all } \xi \in \mathfrak{m},
$$

where $c_{i j}^{k}$ are the structure coefficients of Lie algebra $\mathfrak{m}$, i.e., $\left[e_{i}, e_{j}\right]_{\mathfrak{m}}=\sum_{k} c_{i j}^{k} e_{k}$. Therefore the Poisson bracket on $\mathbb{R}^{n}>\mathfrak{m}^{*}$ is given by

$$
\{f, h\}=0, \quad\{\xi, \eta\}=[\xi, \eta]_{\mathfrak{m}}, \quad\{\xi, f\}=\tilde{\xi} f=\sum_{i, j, k}\left\langle f^{i}, \xi\right\rangle c_{i j}^{k} x^{k} \frac{\partial f}{\partial x^{j}},
$$

where $f, h \in C^{\infty}\left(\mathbb{R}^{n}\right)$ and $\xi, \eta \in \mathfrak{m}$.

The bicrossproduct Hopf algebra $\mathbb{C}[\bar{G}] \bowtie U_{\lambda}\left(\overline{\mathfrak{g}^{*}}\right)=\mathbb{C}\left[\mathbb{R}^{n}\right] \triangleleft U_{\lambda}(\mathfrak{m})$, as the quantisation of $C^{\infty}\left(\mathbb{R}^{n}>\mathfrak{m}^{*}\right)$, has commutation relations

$$
\left[x^{i}, x^{j}\right]=0, \quad\left[e_{i}, e_{j}\right]=\lambda \sum_{k} c_{i j}^{k} e_{k}, \quad\left[e_{i}, x^{j}\right]=\lambda \sum_{k} c_{i j}^{k} x^{k},
$$


where $\left\{x^{i}\right\}$ are coordinate functions of $\mathbb{R}^{n}=\overline{\mathfrak{m}^{*}}$, identified via the basis $\left\{e_{i}\right\}$ of $\mathfrak{m}$. As an algebra we can equally well take $C^{\infty}\left(\mathbb{R}^{n}\right) \rtimes U_{\lambda}(\mathfrak{m})$, i.e., not limiting ourselves to polynomials. Then $\left[e_{i}, f\right]=\lambda \sum_{j, k} c_{i j}^{k} x^{k} \partial f / \partial x^{j}$ more generally for the cross relations.

Example 5.3. We take $\mathrm{SU}_{2}$ with the standard Drinfeld-Sklyanin Lie bialgebra structure on $\mathrm{su}_{2}$, where the matched pair comes from the Iwasawa decomposition of $\mathrm{SL}_{2}(\mathbb{C})$ [Majid 1990a]. The bicrossproduct Hopf algebra $\mathbb{C}\left[\mathrm{SU}_{2}\right] \triangleleft U_{\lambda}\left(\mathrm{su}_{2}^{*}\right)$, as an algebra, is the cross product $\mathbb{C}\left[\mathrm{SU}_{2}\right] \triangleleft U_{\lambda}\left(\mathrm{su}_{2}^{*}\right)$ with $a, b, c, d$ commuting, $a d-b c=1,\left[x^{i}, x^{3}\right]=\lambda x^{i}(i=1,2)$ and

$$
\left[x^{i}, \boldsymbol{t}\right]=\lambda \boldsymbol{t}\left[e_{i}, \boldsymbol{t}^{-1} e_{3} \boldsymbol{t}-e_{3}\right], i=1,2,3,
$$

that is,

$$
\begin{aligned}
& {\left[x^{1}, t\right]=-\lambda b c t e_{2}+\frac{\lambda}{2} t \operatorname{diag}(a c,-b d)+\frac{\lambda}{2} \operatorname{diag}(b,-c),} \\
& {\left[x^{2}, t\right]=\lambda b c t e_{1}-\frac{l \lambda}{2} t \operatorname{diag}(a c, b d)+\frac{l \lambda}{2} \operatorname{diag}(b, c),} \\
& {\left[x^{3}, t\right]=-\lambda a d t+\lambda \operatorname{diag}(a, d),}
\end{aligned}
$$

where $t=\left(\begin{array}{ll}a & b \\ c & d\end{array}\right)$ and $\left\{e_{i}\right\}$ and $\left\{x^{i}\right\}$ are bases of $\mathrm{su}_{2}$ and $\mathrm{su}_{2}^{*}$ as the half-real forms of $\operatorname{sl}_{2}(\mathbb{C})$ and $\mathrm{sl}_{2}^{*}(\mathbb{C})$ respectively. The coalgebra of $\mathbb{C}\left[\mathrm{SU}_{2}\right] \triangleleft U_{\lambda}\left(\mathrm{su}_{2}^{*}\right)$ is the cross coproduct $\mathbb{C}\left[\mathrm{SU}_{2}\right] \propto U_{\lambda}\left(\mathrm{su}_{2}^{*}\right)$ associated with

$$
\Delta\left(x^{i}\right)=1 \otimes x^{i}-2 \sum_{k} x^{k} \otimes \operatorname{Tr}\left(\boldsymbol{t} e_{i} \boldsymbol{t}^{-1} e_{k}\right), \quad \epsilon\left(x^{i}\right)=0 \quad \text { for all } i \in\{1,2,3\} .
$$

The $*$-structure is the known one on $\mathbb{C}\left[\mathrm{SU}_{2}\right]$ with $x^{i *}=-x^{i}$ for each $i$.

Proof. We recall that the coordinate algebra $\mathbb{C}\left[\mathrm{SU}_{2}\right]$ is the commutative algebra $\mathbb{C}[a, b, c, d]$ modulo the relation $a d-b c=1$ with $*$-structure

$$
\left(\begin{array}{ll}
a^{*} & b^{*} \\
c^{*} & d^{*}
\end{array}\right)=\left(\begin{array}{rr}
d & -c \\
-b & a
\end{array}\right)
$$

As a Hopf $*$-algebra, the coproduct, counit and antipode of $\mathbb{C}\left[\mathrm{SU}_{2}\right]$ are given by $\Delta\left(\begin{array}{ll}a & b \\ c & d\end{array}\right)=\left(\begin{array}{ll}a & b \\ c & d\end{array}\right) \otimes\left(\begin{array}{ll}a & b \\ c & d\end{array}\right), \quad \epsilon\left(\begin{array}{ll}a & b \\ c & d\end{array}\right)=\left(\begin{array}{ll}1 & 0 \\ 0 & 1\end{array}\right), \quad S\left(\begin{array}{ll}a & b \\ c & d\end{array}\right)=\left(\begin{array}{rr}d & -b \\ -c & a\end{array}\right)$.

Let $\left\{H, X_{ \pm}\right\}$and $\left\{\phi, \psi_{ \pm}\right\}$be the dual bases of $\operatorname{sl}_{2}(\mathbb{C})$ and $\mathrm{sl}_{2}^{*}(\mathbb{C})$ respectively, where

$$
H=\left(\begin{array}{rr}
1 & 0 \\
0 & -1
\end{array}\right), \quad X_{+}=\left(\begin{array}{ll}
0 & 1 \\
0 & 0
\end{array}\right) \quad \text { and } \quad X_{-}=\left(\begin{array}{ll}
0 & 0 \\
1 & 0
\end{array}\right)
$$


As the half-real forms of $\mathrm{sl}_{2}(\mathbb{C})$ and $\mathrm{sl}_{2}^{*}(\mathbb{C})$, the Lie algebras $\mathrm{su}_{2}$ and $\mathrm{su}_{2}^{*}$ have bases

$$
\begin{array}{cl}
e_{1}=-\frac{1}{2} \imath\left(X_{+}+X_{-}\right), & e_{2}=-\frac{1}{2}\left(X_{+}-X_{-}\right), \quad e_{3}=-\frac{1}{2} \iota H, \\
x^{1}=\psi_{+}+\psi_{-}, & x^{2}=l\left(\psi_{+}-\psi_{-}\right), \quad x^{3}=2 \phi,
\end{array}
$$

respectively. Note that $x^{i}=-\imath f^{i}$, where $\left\{f^{i}\right\}$ is the dual basis of $\left\{e_{i}\right\}$.

The Lie brackets and Lie cobrackets of $\mathrm{su}_{2}$ and $\mathrm{su}_{2}^{*}$ are given by

$$
\begin{gathered}
{\left[e_{i}, e_{j}\right]=\epsilon_{i j k} e_{k} \quad \text { and } \quad \delta e_{i}=\imath e_{i} \wedge e_{3} \quad \text { for all } i, j, k,} \\
{\left[x^{1}, x^{2}\right]=0, \quad\left[x^{i}, x^{3}\right]=x^{i}, i=1,2, \quad \delta x^{1}=\imath\left(x^{2} \otimes x^{3}-x^{3} \otimes x^{2}\right),} \\
\delta x^{2}=l\left(x^{3} \otimes x^{1}-x^{1} \otimes x^{3}\right), \quad \delta x^{3}=l\left(x^{1} \otimes x^{2}-x^{2} \otimes x^{1}\right),
\end{gathered}
$$

where $\epsilon_{i j k}$ is totally antisymmetric and $\epsilon_{123}=1$. Writing $\xi=\xi^{i} e_{i} \in \mathrm{su}_{2}$ and $\phi=\phi_{i} x^{i} \in \mathrm{su}_{2}^{*}$ for 3 -vectors $\vec{\xi}=\left(\xi^{i}\right), \vec{\phi}=\left(\phi_{i}\right)$, we know that $\left(\mathrm{su}_{2}^{*}, \mathrm{su}_{2}\right)$ forms a the matched pair of Lie bialgebras with interacting actions

$$
\vec{\xi} \triangleleft \vec{\phi}=\left(\vec{\xi} \times \overrightarrow{e_{3}}\right) \times \vec{\phi}, \quad \vec{\xi} \triangleright \vec{\phi}=\vec{\xi} \times \vec{\phi}
$$

To obtain the action of $\mathrm{su}_{2}^{*}$ on $\mathbb{C}\left[\mathrm{SU}_{2}\right]$, we need to solve [Majid 1995, Proposition 8.3.14]

$$
\left.\frac{\mathrm{d}}{\mathrm{d} t} a_{\phi}\left(\mathrm{e}^{t \xi} u\right)\right|_{t=0}=\operatorname{Ad}_{u^{-1}}(\xi \triangleleft(u \triangleright \phi)), \quad a_{\phi}\left(I_{2}\right)=0 .
$$

Note that $\mathrm{SU}_{2}$ acts on $\mathrm{su}_{2}^{*}$ by $u \triangleright \vec{\phi}=\operatorname{Rot}_{u} \vec{\phi}$, where we view $\phi$ as an element in $\mathrm{su}_{2}$ via $\rho(\phi)=\phi_{i} e_{i}$. One can check that

$$
a_{\vec{\phi}}(u)=\vec{\phi} \times\left(\operatorname{Rot}_{u^{-1}}\left(\overrightarrow{e_{3}}\right)-\vec{e}_{3}\right)
$$

is the unique solution to the differential equation. Now we can compute by (5-3)

$$
\begin{aligned}
\left(\phi \triangleright \boldsymbol{t}_{j}^{i}\right)(u) & =\left.\frac{\mathrm{d}}{\mathrm{d} t} \boldsymbol{t}_{j}^{i}\left(u \mathrm{e}^{t a_{\phi}(u)}\right)\right|_{t=0} \\
& =\left.\sum_{k} \frac{\mathrm{d}}{\mathrm{d} t} \boldsymbol{t}_{k}^{i}(u) \boldsymbol{t}_{j}^{k}\left(\mathrm{e}^{t a_{\phi}(u)}\right)\right|_{t=0} \\
& =\sum_{k} u_{k}^{i}\left(a_{\phi}(u)\right)_{j}^{k} \\
& =\sum_{k} u_{k}^{i}\left[\rho(\phi), u^{-1} e_{3} u-e_{3}\right]_{j}^{k},
\end{aligned}
$$

where $\rho(\phi)=\sum_{i} \phi_{i} e_{i}$. This shows that

$$
\left[x^{i}, \boldsymbol{t}\right]=\lambda x^{i} \triangleright \boldsymbol{t}=\lambda \boldsymbol{t}\left[e_{i}, \boldsymbol{t}^{-1} e_{3} \boldsymbol{t}-e_{3}\right],
$$


as displayed. For each $i$, we can work out the terms on the right explicitly (using $a d-b c=1)$ as

$$
\begin{aligned}
& {\left[x^{1}, t\right]=-\frac{\lambda}{2}\left(\begin{array}{cc}
a b d-a^{2} c-2 b, & b^{2} d-a^{2} d+a \\
a d^{2}-a c^{2}-d, & b d^{2}-a c d+2 c
\end{array}\right),} \\
& {\left[x^{2}, t\right]=-\frac{\imath \lambda}{2}\left(\begin{array}{cc}
a^{2} c+a b d-2 b, & a^{2} d+b^{2} d-a \\
a c^{2}+a d^{2}-d, & b d^{2}+a c d-2 c
\end{array}\right),} \\
& {\left[x^{3}, t\right]=-\lambda\left(\begin{array}{cc}
a^{2} d-a, & a b d \\
a c d, & a d^{2}-d
\end{array}\right) .}
\end{aligned}
$$

These can be rewritten as the formulae (5-8) we stated.

For convenience, we use Pauli matrices $\sigma_{1}=\left(\begin{array}{ll}0 & 1 \\ 1 & 0\end{array}\right), \sigma_{2}=\left(\begin{array}{cc}0 & -l \\ l & 0\end{array}\right), \sigma_{3}=\left(\begin{array}{cc}1 & 0 \\ 0 & -1\end{array}\right)$. Clearly, $e_{i}=-\frac{1}{2} \iota \sigma_{i}$ and $\sigma_{i}$ obey $\sigma_{i} \sigma_{j}=\delta_{i j} I_{2}+\imath \epsilon_{i j k} \sigma_{k}$ and $\left[\sigma_{i}, \sigma_{j}\right]=2 \imath \epsilon_{i j k} \sigma_{k}$.

The coaction of $\mathbb{C}\left[\mathrm{SU}_{2}\right]$ on $\mathrm{su}_{2}^{*}$ is defined by $\beta(\phi)(u)=u \triangleright \phi=\operatorname{Rot}_{u} \vec{\phi}$ for any $u \in \mathrm{SU}_{2}, \phi \in \mathrm{su}_{2}^{*}$. Again, we view $\phi$ as an element in $\mathrm{su}_{2}$, so $\rho(u \triangleright \phi)=u \rho(\phi) u^{-1}$, namely $\sum_{i}(u \triangleright \phi)_{i} \sigma_{i}=\sum_{i} \phi_{i} u \sigma_{i} u^{-1}$. In particular, we have

$$
\left(u \triangleright x^{i}\right)_{1} \sigma_{1}+\left(u \triangleright x^{i}\right)_{2} \sigma_{2}+\left(u \triangleright x^{i}\right)_{3} \sigma_{3}=u \sigma_{i} u^{-1}, \quad i=1,2,3 .
$$

Multiplying by $\sigma_{k}$ on the right and then taking the trace of both sides, and using $\operatorname{Tr}\left(\sigma_{i} \sigma_{j}\right)=2 \delta_{i j}$ we have $2\left(u \triangleright x^{i}\right)_{k}=\operatorname{Tr}\left(u \sigma_{i} u^{-1} \sigma_{k}\right)$. Therefore

$$
u \triangleright x^{i}=\frac{1}{2} \sum_{k} \operatorname{Tr}\left(u \sigma_{i} u^{-1} \sigma_{k}\right) x^{k}=-2 \sum_{k} \operatorname{Tr}\left(u e_{i} u^{-1} e_{k}\right) x^{k},
$$

and thus $\beta\left(x^{i}\right)=\frac{1}{2} \sum_{k} x^{k} \otimes \operatorname{Tr}\left(\boldsymbol{t} \sigma_{i} \boldsymbol{t}^{-1} \sigma_{k}\right)=-2 \sum_{k} x^{k} \otimes \operatorname{Tr}\left(\boldsymbol{t} e_{i} \boldsymbol{t}^{-1} e_{k}\right)$. This gives rise to the coproduct of $x^{i}$ as stated. This example is dual to a bicrossproduct from this matched pair computed in [Majid 1995].

5C. Preconnections on the tangent bundle $\bar{G} \triangleright \backslash \mathfrak{g}$. We use the following lemma

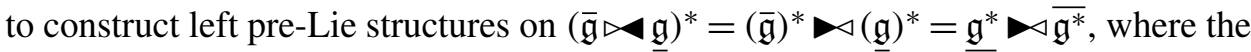
Lie bracket is the semidirect sum $\mathfrak{g}^{*} \rtimes \mathfrak{g}^{*}$ and the Lie cobracket is the semidirect cobracket $\mathfrak{g}^{*}<\overline{\mathfrak{g}^{*}}$, namely

$$
\begin{gathered}
{[\phi, \psi]=0, \quad[f, \phi]=f \triangleright \phi=[f, \phi]_{\mathfrak{g}^{*}}, \quad[f, g]=[f, g]_{\mathfrak{g}^{*}},} \\
\delta \phi=\delta_{\mathfrak{g}^{*}} \phi=\phi_{(1)} \otimes \phi_{(2)}, \quad \delta f=\underline{f_{(1)}} \otimes \overline{f_{(2)}}-\underline{f_{(2)}} \otimes \overline{f_{(1)}},
\end{gathered}
$$

for any $\phi, \psi \in \underline{\mathfrak{g}^{*}}, f, g \in \overline{\mathfrak{g}^{*}}$. For convenience, we denote $f \in \mathfrak{g}^{*}$ by $\bar{f}$ if viewed in $\overline{\mathfrak{g}^{*}}$ or $\underline{f}$ if viewed in $\underline{\mathfrak{g}}^{*}$.

Lemma 5.4. Let $(A, \circ)$ be a left pre-Lie algebra and $(B, *)$ a left pre-Lie algebra in the category $\mathfrak{g}_{A}$ M of left $\mathfrak{g}_{A}$-modules, i.e., there is a left $\mathfrak{g}_{A}$-action $\triangleright$ on $B$ such that

$$
a \triangleright(x * y)=(a \triangleright x) * y+x *(a \triangleright y)
$$


for any $a, b \in A, x, y \in B$. Then there is a left pre-Lie algebra structure on $B \oplus A$

$$
(x, a) \tilde{\circ}(y, b)=(x * y+a \triangleright y, a \circ b) .
$$

We denote this pre-Lie algebra by $B \rtimes A$, and have $\mathfrak{g}_{B \rtimes A}=\mathfrak{g}_{B} \rtimes \mathfrak{g}_{A}$ for the associated Lie algebras.

Proof. This is a matter of directly verifying according to the axioms of a left pre-Lie algebra.

Corollary 5.5. Let $(\mathfrak{m}, \circ)$ be a left pre-Lie algebra. Suppose it admits a (not necessarily unital) commutative associative product $\cdot$ such that

$$
[\xi, x \cdot y]_{\mathfrak{m}}=[\xi, x]_{\mathfrak{m}} \cdot y+x \cdot[\xi, \eta]_{\mathfrak{m}} \quad \text { for all } \xi, x, y \in \mathfrak{m},
$$

where $[,]_{\mathfrak{m}}$ is the Lie bracket defined by $\circ$. Denote the underlying pre-Lie algebra by $\underline{\mathfrak{m}}=(\mathfrak{m}, \cdot)$. Then $\underline{\mathfrak{m}} \rtimes_{\mathrm{ad}} \mathfrak{m}$ is a left pre-Lie algebra with product

$$
(x, \xi) \tilde{\circ}(y, \eta)=\left(x \cdot y+[\xi, y]_{\mathfrak{m}}, \xi \circ \eta\right)
$$

for any $x, y \in \underline{\mathfrak{m}}, \xi, \eta \in \mathfrak{m}$.

Proof. Take $(A, \circ)=(\mathfrak{m}, \circ)$ and $(B, *)=(\mathfrak{m}, \cdot)$ in Lemma 5.4. Here $(\mathfrak{m}, \circ)$ left acts on $(\mathfrak{m}, \cdot)$ by the adjoint action and (5-9) is exactly the condition displayed.

The assumption made in Corollary 5.5 is that $(\mathfrak{m}, \cdot,[]$,$) is a (not necessarily$ unital) Poisson algebra with respect to the Lie bracket, and that the latter admits a left pre-Lie structure $\circ$.

Theorem 5.6. Let $G$ be a finite-dimensional connected and simply connected Poisson-Lie group with Lie bialgebra $\mathfrak{g}$. Assume that $\left(\mathfrak{g}^{*},[,]_{\mathfrak{g}^{*}}\right)$ admits a preLie structure $\circ$ and also that $\mathfrak{g}^{*}$ admits a (not necessarily unital) Poisson algebra structure $\left(\mathfrak{g}^{*}, *,[,]_{\mathfrak{g}^{*}}\right)$

$$
[f, \phi * \psi]_{\mathfrak{g}^{*}}=[f, \phi]_{\mathfrak{g}^{*}} * \psi+\phi *[f, \psi]_{\mathfrak{g}^{*}}
$$

for any $\phi, \psi \in \underline{\mathfrak{g}^{*}}, f \in \mathfrak{g}^{*}$. Then the semidirect sum $\underline{\mathfrak{g}^{*}} \rtimes \mathfrak{g}^{*}$ admits a pre-Lie algebra product õ given by

$$
(\phi, f) \tilde{\circ}(\psi, h)=\left(\phi * \psi+[f, \psi]_{\mathfrak{g}^{*}}, f \circ h\right),
$$

and the tangent bundle $\bar{G} \bowtie \underline{\mathfrak{g}}$ in Lemma 5.1 admits a Poisson-compatible leftcovariant flat preconnection.

Proof. We take $\mathfrak{m}=\mathfrak{g}^{*}$ in Corollary 5.5. We know $\mathfrak{g}^{*} \rtimes \mathfrak{g}^{*}$ is the Lie algebra of $\mathfrak{g}^{*} \triangleleft \overline{\mathfrak{g}^{*}}$, dual to Lie algebra $\overline{\mathfrak{g}} \bowtie \mathfrak{g}$ of the tangent bundle. Then we apply Corollary 4.2. 
The corresponding preconnection can be computed explicitly from (2-8). For a Poisson-Lie group $G$, let $\left\{e_{i}\right\}$ be a basis of $\mathfrak{g}$ and $\left\{f^{i}\right\}$ the dual basis of $\mathfrak{g}^{*}$. Denote by $\left\{\omega^{i}\right\}$ the basis of left-invariant 1-forms that is dual to $\left\{\partial_{i}\right\}$ the left-invariant vector fields of $G$ generated by $\left\{e_{i}\right\}$ as before. For the abelian Poisson-Lie group $\mathfrak{g}$ with Kirillov-Kostant Poisson bracket, let $\left\{E_{i}\right\}$ be a basis of $\mathfrak{g}$ and $\left\{x^{i}\right\}$ the dual basis of $\overline{\mathfrak{g}^{*}}$. Then $\left\{\mathrm{d} x^{i}\right\}$ is the basis of left-invariant 1 -forms that is dual to $\left\{\partial / \partial x^{i}\right\}$, the basis of the left-invariant vector fields on $\mathfrak{g}$ generated by $\left\{E_{i}\right\}$. Now we can choose $\left\{e_{i}, E_{i}\right\}$ to be the basis of $\overline{\mathfrak{g}} \bowtie \mathfrak{g}$, and so $\left\{f^{i}, x^{i}\right\}$ is the dual basis for $\mathfrak{g}^{*} \triangleleft \overline{\mathfrak{g}^{*}}$. Denote by $\left\{\tilde{\partial}_{i}, D_{i}\right\}$ the left-invariant vector fields on $\bar{G} \bowtie \mathfrak{g}$ generated by $\left\{e_{i}, E_{i}\right\}$, and denote by $\left\{\tilde{\omega}^{i}, \widetilde{\mathrm{dx}}{ }^{i}\right\}$ the corresponding dual basis of left-invariant 1-forms. By construction, when viewing any $f \in C^{\infty}(G)$ and $\phi \in \mathfrak{g}^{*} \subset C^{\infty}(\mathfrak{g})$ as functions on the tangent bundle, we know

$$
\tilde{\partial}_{i} f=\partial_{i} f, \quad \tilde{\partial}_{i} \phi=\operatorname{ad}_{e_{i}}^{*} \phi, \quad D_{i} f=0, \quad D_{i} \phi=\frac{\partial}{\partial x^{i}} \phi .
$$

This implies

$\tilde{\partial}_{i}=\partial_{i}+\sum_{j}\left(\operatorname{ad}_{e_{i}}^{*} x^{j}\right) \frac{\partial}{\partial x^{j}}, \quad D_{i}=\frac{\partial}{\partial x^{i}}, \quad \tilde{\omega}^{i}=\omega^{i}, \quad \widetilde{\mathrm{d} x^{i}}=\mathrm{d} x^{i}-\sum_{k}\left(\operatorname{ad}_{e_{k}}^{*} x^{i}\right) \omega^{k}$.

Let $\tilde{o}$ be the pre-Lie structure of $\mathfrak{g}^{*} \rtimes \mathfrak{g}^{*}$ constructed by (5-12) in terms of $*$ and $\circ$ in the setting of Theorem 5.6. The Poisson-compatible left-covariant flat preconnection on the tangent bundle is then, for any function $a$,

$$
\begin{aligned}
\gamma\left(a, \omega^{j}\right) & =\sum_{i, k} \widetilde{\partial_{i}} a\left\langle f^{i} * f^{j}, e_{k}\right\rangle \omega^{k}+\sum_{i, k} D_{i} a\left\langle\left[x^{i}, f^{j}\right]_{\mathfrak{g}^{*}}, e_{k}\right\rangle \omega^{k}, \\
\gamma\left(a, \widetilde{\mathrm{d} x^{j}}\right) & =\sum_{i, k} D_{i} a\left\langle x^{i} \circ x^{j}, E_{k}\right\rangle \widetilde{\mathrm{d} x^{k}} .
\end{aligned}
$$

If we write

$$
\begin{gathered}
f^{i} * f^{j}=\sum_{k} a_{k}^{i j} f^{k}, \quad x^{i} \circ x^{j}=\sum_{k} b_{k}^{i j} x^{k} \\
{\left[x^{i}, f^{j}\right]_{\mathfrak{g}^{*}}=\sum_{k}\left\langle\left[x^{i}, f^{j}\right]_{\mathfrak{g}^{*}}, e_{k}\right\rangle f^{k}=\sum_{s, k} d_{k}^{s j}\left\langle x^{i}, e_{s}\right\rangle f^{k},}
\end{gathered}
$$

where $\left[f^{i}, f^{j}\right]_{\mathfrak{g}^{*}}=d_{k}^{i j} f^{k}$, then the left-covariant preconnection on the tangent bundle $\bar{G} \triangleright \underline{\mathfrak{g}}$ is

$$
\begin{gathered}
\gamma\left(f, \omega^{j}\right)=\sum_{i, k} a_{k}^{i j}\left(\partial_{i} f\right) \omega^{k}, \quad \gamma\left(f, \widetilde{\mathrm{d} x^{j}}\right)=0, \quad \gamma\left(\phi, \widetilde{\mathrm{d} x^{j}}\right)=\sum_{i, k} b_{k}^{i j}\left(\frac{\partial \phi}{\partial x^{i}}\right) \widetilde{\mathrm{d} x^{k}}, \\
\gamma\left(\phi, \omega^{j}\right)=\sum_{i, k}\left(a_{k}^{i j} \operatorname{ad}_{e_{i}}^{*} \phi+\sum_{s} d_{k}^{s j}\left\langle x^{i}, e_{s}\right\rangle\left(\frac{\partial \phi}{\partial x^{i}}\right)\right) \omega^{k}
\end{gathered}
$$

for any $f \in C^{\infty}(G), \phi \in \mathfrak{g}^{*} \subset C^{\infty}(\mathfrak{g})$. 
This result applies, for example, to tell us that we have a left-covariant differential structure on quantum groups such as $\mathbb{C}[\bar{G}] \triangleleft U_{\lambda}\left(\mathfrak{g}^{*}\right)$ at least to lowest order in deformation. In the special case when the product $*$ is zero, there is a natural differential calculus not only at lowest order. Under the notations above, we have:

Proposition 5.7. Let $G$ be a finite-dimensional connected and simply connected Poisson-Lie group with Lie algebra $\mathfrak{g}$. If the dual Lie algebra $\mathfrak{g}^{*}$ admits a preLie structure $\circ: \mathfrak{g}^{*} \otimes \mathfrak{g}^{*} \rightarrow \mathfrak{g}^{*}$ with respect to its Lie bracket $\left([,]_{\mathfrak{g}^{*}}\right.$ determined by $\delta_{\mathfrak{g}}$ ), then the bicrossproduct $\mathbb{C}[\bar{G}] \bowtie U_{\lambda}\left(\mathfrak{g}^{*}\right)$ (if it exists) admits a left-covariant differential calculus

$$
\Omega^{1}=\left(\mathbb{C}[\bar{G}] \bowtie U_{\lambda}\left(\mathfrak{g}^{*}\right)\right) \ltimes \Lambda^{1}
$$

with left-invariant 1-forms $\Lambda^{1}$ spanned by basis $\left\{\omega^{i}, \widetilde{\mathrm{d} x}{ }^{i}\right\}$, where the commutation relations and the derivatives are given by

$$
\begin{gathered}
{\left[f, \omega^{i}\right]=0, \quad\left[f, \widetilde{\mathrm{d} x^{i}}\right]=0, \quad\left[x^{i}, \omega^{j}\right]=\sum_{k} \lambda\left\langle\left[x^{i}, f^{j}\right]_{\mathfrak{g}^{*}}, e_{k}\right\rangle \omega^{k},} \\
{\left[x^{i}, \widetilde{\mathrm{d} x^{j}}\right]=\lambda \mathrm{d}\left(\widetilde{x^{i} \circ x^{j}}\right), \quad \mathrm{d} f=\sum_{j}\left(\partial_{j} f\right) \omega^{j}, \quad \mathrm{~d} x^{i}=\widetilde{\mathrm{d} x^{i}}+\sum_{j}\left(\operatorname{ad}_{e_{j}}^{*} x^{i}\right) \omega^{j}}
\end{gathered}
$$

for any $f \in \mathbb{C}[\bar{G}]$.

Proof. It is easy to see that we have a bimodule $\Omega^{1}$. As the notation indicates [Majid and Tao 2015b], the left action on $\Omega^{1}$ is the product of the bicrossproduct quantum group on itself while the right action is the tensor product of the right action of the bicrossproduct on itself and a right action on $\Lambda^{1}$. The right action of $\mathbb{C}[G]$ here is trivial, namely

$$
\omega^{j} \triangleleft f=f(e) \omega^{j}, \quad \widetilde{\mathrm{d} x^{j}} \triangleleft f=f(e) \widetilde{\mathrm{d} x^{j}} ;
$$

the right actions of $x^{i}$ are clear from the commutation relations and given (summation understood) by

$$
\begin{aligned}
& \omega^{j} \triangleleft x^{i}=-\lambda\left\langle\left[x^{i}, f^{j}\right]_{\mathfrak{g}^{*}}, e_{k}\right\rangle \omega^{k}=-\lambda d_{k}^{s j}\left\langle x^{i}, e_{s}\right\rangle \omega^{k}, \\
& \widetilde{\mathrm{d} x^{j}} \triangleleft x^{i}=-\lambda \mathrm{d}\left(\widetilde{x^{i} \circ x^{j}}\right)=-\lambda b_{k}^{i j} \widetilde{\mathrm{d} x^{k}} .
\end{aligned}
$$

One can check that these fit together to a right action of the bicrossproduct quantum group by using the Jacobi identity of $\mathfrak{g}^{*}$, the pre-Lie identity on $\circ$, and the fact that $\left(\widetilde{x^{i}} f\right)(e)=\widetilde{x_{e}^{i}} f=0$ by $(5-6)$.

We check that the Leibniz rule holds. The conditions

$$
\mathrm{d}[f, h]=0 \quad \text { and } \quad \mathrm{d}\left[x^{i}, x^{j}\right]=\lambda \mathrm{d}\left[x^{i}, x^{j}\right]_{\mathfrak{g}^{*}}
$$

are easy to check, so we omit these. It remains to check that

$$
\mathrm{d}\left[x^{i}, f\right]=\lambda \mathrm{d}\left(\tilde{x^{i}} f\right) \text { for all } f \in \mathbb{C}[\bar{G}] .
$$


The right-hand side of (5-13) is

$$
\lambda \mathrm{d}\left(\tilde{x^{i}} f\right)=\lambda \partial_{j}\left(\widetilde{x^{i}} f\right) \omega^{j},
$$

while the left-hand side of (5-13) is

$$
\begin{aligned}
\mathrm{d}\left[x^{i}, f\right] & =\mathrm{d}\left(x^{i} f-x^{i} f\right)=\left[\mathrm{d} x^{i}, f\right]+\left[x^{i}, \mathrm{~d} f\right] \\
& =\left[\widetilde{\mathrm{d} x^{i}}+\left(\operatorname{ad}_{e_{j}}^{*} x^{i}\right) \omega^{j}, f\right]+\left[x^{i},\left(\partial_{j} f\right) \omega^{j}\right] \\
& =0+\left[\operatorname{ad}_{e_{j}}^{*} x^{i}, f\right] \omega^{j}+\left[x^{i}, \partial_{j} f\right] \omega^{j}+\left(\partial_{k} f\right)\left[x^{i}, \omega^{k}\right] \\
& =\left[\operatorname{ad}_{e_{j}}^{*} x^{i}, f\right] \omega^{j}+\left[x^{i}, \partial_{j} f\right] \omega^{j}+\lambda\left(\partial_{k} f\right)\left\langle\left[x^{i}, f^{k}\right]_{\mathfrak{g}^{*}}, e_{j}\right\rangle \omega^{j} \\
& =\left(\left[\operatorname{ad}_{e_{j}}^{*} x^{i}, f\right]+\left[x^{i}, \partial_{j} f\right]+\lambda\left\langle\left[x^{i}, f^{k}\right]_{\mathfrak{g}^{*}}, e_{j}\right\rangle\left(\partial_{k} f\right)\right) \omega^{j} \\
& =\lambda\left({\widetilde{\operatorname{ad}_{e_{j}}^{*}} x^{i}}_{f}^{x^{i}}\left(\partial_{j} f\right)+\left\langle\left[x^{i}, f^{k}\right]_{\mathfrak{g}^{*}}, e_{j}\right\rangle\left(\partial_{k} f\right)\right) \omega^{j} .
\end{aligned}
$$

It suffices to show that $\partial_{j}\left(\tilde{x^{i}} f\right)=\widetilde{\operatorname{ad}_{e_{j}}^{*} x^{i}} f+\widetilde{x^{i}}\left(\partial_{j} f\right)+\left\langle\left[x^{i}, f^{k}\right]_{\mathfrak{g}^{*}}, e_{j}\right\rangle\left(\partial_{k} f\right)$, namely

$$
\left[\partial_{j}, \widetilde{x^{i}}\right]=\widetilde{\operatorname{ad}_{e_{j}}^{*} x^{i}}+\left\langle\left[x^{i}, f^{k}\right]_{\mathfrak{g}^{*}}, e_{j}\right\rangle \partial_{k} .
$$

Recall that in the double cross-sum $\mathfrak{g}^{*} \bowtie \mathfrak{g}$, for any $e_{j} \in \mathfrak{g}, x^{i} \in \mathfrak{g}^{*}$,

$$
\left[e_{j}, x^{i}\right]=e_{j} \triangleleft x^{i}+e_{j} \triangleright x^{i}=\left\langle\left[x^{i}, f^{k}\right]_{\mathfrak{g}^{*}}, e_{j}\right\rangle e_{k}+\operatorname{ad}_{e_{j}}^{*} x^{i} .
$$

Therefore the condition left to check is nothing but the Lie bracket of elements $e_{j}$ and $x^{i}$ viewed as the infinitesimal action of $\mathfrak{g}^{*} \bowtie \mathfrak{g}$ on $\mathbb{C}[\bar{G}]$, as explained in the general theory of double cross-sums in Section 5A.

Now we compute the left-covariant first-order differential calculus on the bicrossproduct quantum group $\mathbb{C}\left[\mathrm{SU}_{2}\right] \triangleleft U_{\lambda}\left(\mathrm{su}_{2}^{*}\right)$ constructed in Example 5.3.

Example 5.8. As in Example 4.6, the classical connected left-covariant calculus on $\mathbb{C}\left[\mathrm{SU}_{2}\right]$ has basis of left-invariant 1-forms

$$
\omega^{0}=d \mathrm{~d} a-b \mathrm{~d} c=c \mathrm{~d} b-a \mathrm{~d} d, \quad \omega^{+}=d \mathrm{~d} b-b \mathrm{~d} d, \quad \omega^{-}=a \mathrm{~d} c-c \mathrm{~d} a
$$

(corresponding to the Chevalley basis $\left\{H, X_{ \pm}\right\}$of $\mathrm{su}_{2}$ ) with exterior derivative

$$
\mathrm{d} a=a \omega^{0}+b \omega^{-}, \quad \mathrm{d} b=a \omega^{+}-b \omega^{0}, \quad \mathrm{~d} c=c \omega^{0}+d \omega^{-}, \quad \mathrm{d} d=c \omega^{+}-d \omega^{0} .
$$

Let $\circ: \mathrm{su}_{2}^{*} \otimes \mathrm{su}_{2}^{*} \rightarrow \mathrm{su}_{2}^{*}$ be a left pre-Lie algebra structure of $\mathrm{su}_{2}^{*}$ with respect to the Lie bracket $\left[x^{1}, x^{2}\right]=0$ and $\left[x^{i}, x^{3}\right]=x^{i}$ for $i=1,2$. Let

$$
\left\{\widetilde{\mathrm{d} x^{1}}, \widetilde{\mathrm{d} x^{2}}, \widetilde{\mathrm{d} x^{3}}\right\}
$$

complete the basis of left-invariant 1-forms on the tangent bundle as explained above. According to Proposition 5.7, this defines a 6-dimensional connected left-covariant 
differential calculus on the bicrossproduct $\mathbb{C}\left[\mathrm{SU}_{2}\right] \triangleleft U_{\lambda}\left(\mathrm{su}_{2}^{*}\right)$ with commutation relations and exterior derivative given by

$$
\begin{gathered}
{\left[\boldsymbol{t}, \omega^{l}\right]=0 \quad \text { for all } l \in\{0, \pm\}, \quad\left[\boldsymbol{t}, \widetilde{\mathrm{d} x^{i}}\right]=0,} \\
{\left[x^{i}, \widetilde{\mathrm{d} x^{j}}\right]=\lambda \mathrm{d}\left(\widetilde{x^{i} \circ x^{j}}\right) \quad \text { for all } i, j \in\{1,2,3\},} \\
{\left[x^{1}, \omega^{0}\right]=\frac{1}{2} \lambda\left(\omega^{+}+\omega^{-}\right), \quad\left[x^{1}, \omega^{+}\right]=0, \quad\left[x^{1}, \omega^{-}\right]=0,} \\
{\left[x^{2}, \omega^{0}\right]=\frac{1}{2} \iota \lambda\left(\omega^{+}-\omega^{-}\right), \quad\left[x^{2}, \omega^{+}\right]=0, \quad\left[x^{2}, \omega^{-}\right]=0,} \\
{\left[x^{3}, \omega^{0}\right]=0, \quad\left[\begin{array}{ll}
\left.x^{3}, \omega^{+}\right]=-\lambda \omega^{+}, & {\left[x^{3}, \omega^{-}\right]=-\lambda \omega^{-},} \\
\mathrm{d} x^{1}=\widetilde{\mathrm{d} x^{1}}+2 l x^{2} \omega^{0}+x^{3} \omega^{+}-x^{3} \omega^{-}, & \mathrm{d} x^{2}=\widetilde{\mathrm{d} x^{2}-2 \imath x^{1}} \omega^{0}+l x^{3} \omega^{+}+\imath x^{3} \omega^{-}, \\
c & b \\
c & d
\end{array}\right)=\left(\begin{array}{ll}
a & b \\
c & d
\end{array}\right)\left(\begin{array}{cc}
\omega^{0} & \omega^{+} \\
\omega^{-} & -\omega^{0}
\end{array}\right),} \\
\mathrm{d} x^{3}=\widetilde{\mathrm{d} x^{3}}-\left(x^{1}+l x^{2}\right) \omega^{+}+\left(x^{1}-\imath x^{2}\right) \omega^{-} .
\end{gathered}
$$

Proof. The commutation relations and derivative are computed from the formulae provided in Proposition 5.7. It is useful to also provide an independent, more algebraic proof of the example from [Majid and Tao 2015b, Theorem 2.5], where left-covariant first-order differential calculi $\Omega^{1}$ over a Hopf algebra $A$ are constructed from pairs $\left(\Lambda^{1}, \omega\right)$ where $\Lambda^{1}$ is a right $A$-module and $\omega: A^{+} \rightarrow \Lambda^{1}$ is a surjective right $A$-module map. Given such a pair, the commutation relation and derivative are given by $[a, v]=a v-a_{(1)} v \triangleleft a_{(2)}$ and $\mathrm{d} a=a_{(1)} \otimes \omega \pi_{\epsilon}\left(a_{(2)}\right)$ for any $a \in A$ and $v \in \Lambda^{1}$, where $\pi_{\epsilon}=\mathrm{id}-1 \epsilon$ and $\epsilon$ is the counit.

Firstly, the classical calculus on $A:=\mathbb{C}\left[\mathrm{SU}_{2}\right]$ corresponds to a pair $\left(\Lambda_{A}^{1}, \omega_{A}\right)$ with $\Lambda_{A}^{1}=\operatorname{span}\left\{\omega^{0}, \omega^{ \pm}\right\}$, where the right $\mathbb{C}\left[\mathrm{SU}_{2}\right]$-action on $\Lambda_{A}^{1}$ and the right $\mathbb{C}\left[\mathrm{SU}_{2}\right]$-module surjective map $\omega_{A}: \mathbb{C}\left[\mathrm{SU}_{2}\right]^{+} \rightarrow \Lambda_{A}^{1}$ are given by

$$
\begin{gathered}
\omega^{j} \triangleleft \boldsymbol{t}=\epsilon(\boldsymbol{t}) \omega^{j}, \quad j \in\{0, \pm\}, \\
\omega_{A}\left(\boldsymbol{t}-I_{2}\right)=\omega_{A}\left(\begin{array}{cc}
a-1 & b \\
c & d-1
\end{array}\right)=\left(\begin{array}{cc}
\omega^{0} & \omega^{+} \\
\omega^{-} & -\omega^{0}
\end{array}\right) .
\end{gathered}
$$

Meanwhile, the calculus over $H:=U_{\lambda}\left(\mathrm{su}_{2}^{*}\right)$ corresponds to a pair $\left(\Lambda_{H}^{1}, \omega_{H}\right)$ with

$$
\Lambda_{H}^{1}=\operatorname{span}\left\{\widetilde{\mathrm{d} x^{1}}, \widetilde{\mathrm{d} x^{2}}, \widetilde{\mathrm{d} x^{3}}\right\},
$$

in which the right $U_{\lambda}\left(\mathrm{su}_{2}^{*}\right)$-action on $\Lambda_{H}^{1}$ and the right $U_{\lambda}\left(\mathrm{su}_{2}^{*}\right)$-module surjective map $\omega_{H}: U_{\lambda}\left(\mathrm{su}_{2}^{*}\right)^{+} \rightarrow \Lambda_{H}^{1}$ are given by

$$
\widetilde{\mathrm{d} x^{j}} \triangleleft x^{i}=-\lambda \widetilde{\mathrm{d}\left(\widetilde{x^{i} \circ x^{j}}\right)} \text { and } \omega_{H}\left(x^{i}\right)=\widetilde{\mathrm{d} x^{i}} \quad \text { for all } i, j \in\{1,2,3\} .
$$

Next we construct a pair $\left(\Lambda^{1}, \omega\right)$ over $\widetilde{A}=A \bowtie \triangleleft H$ with direct sum $\Lambda^{1}=\Lambda_{A}^{1} \oplus \Lambda_{H}^{1}$. First, it is clear that $\Lambda_{H}^{1}$ is a right $\widetilde{A}$-module with trivial $A$-action $\mathrm{d} x^{j} \triangleleft t=\epsilon(\boldsymbol{t}) \mathrm{d} x^{j}$, 
One can see this more generally as

$$
v \triangleleft\left(\left(h_{(1)} \triangleright a\right) h_{(2)}\right)=\epsilon(a) v \triangleleft h=(v \triangleleft h) \triangleleft a=v \triangleleft(h a) .
$$

Next, we define a right $U_{\lambda}\left(\mathrm{su}_{2}^{*}\right)$-action on $\Lambda_{A}^{1}$ by the Lie bracket of $\mathrm{su}_{2}^{*}$ viewing $\left\{\omega^{0}, \omega^{ \pm}\right\}$as $\left\{\phi, \psi_{ \pm}\right\}$(the dual basis to $\left\{H, X_{ \pm}\right\}$), where

$$
\left\{x^{1}=\psi_{+}+\psi_{-}, x^{2}=\imath\left(\psi_{+}-\psi_{-}\right), x^{3}=2 \phi\right\}
$$

is the basis for the half-real form $\mathrm{su}_{2}^{*}$ of $\mathrm{sl}_{2}^{*}$, namely

$$
\begin{array}{lll}
\omega^{0} \triangleleft x^{1}=-\frac{1}{2} \lambda\left(\omega^{+}+\omega^{-}\right), & \omega^{+} \triangleleft x^{1}=0, & \omega^{-} \triangleleft x^{1}=0, \\
\omega^{0} \triangleleft x^{2}=-\frac{1}{2} \imath \lambda\left(\omega^{+}-\omega^{-}\right), & \omega^{+} \triangleleft x^{2}=0, & \omega^{-} \triangleleft x^{2}=0, \\
\omega^{0} \triangleleft x^{3}=0, & \omega^{+} \triangleleft x^{3}=\lambda \omega^{+}, & \omega^{-} \triangleleft x^{3}=\lambda \omega^{-} .
\end{array}
$$

This $H$-action commutes with the original trivial $A$-action on $\Lambda_{A}^{1}$, hence $\Lambda_{A}^{1}$ also becomes a right $\widetilde{A}$-module, as does $\Lambda_{A}^{1} \oplus \Lambda_{H}^{1}$.

We then define the map $\omega: \widetilde{A}^{+} \rightarrow \Lambda_{A}^{1} \oplus \Lambda_{H}^{1}$ on generators by $\omega\left(\boldsymbol{t}-I_{2}\right)=\omega_{A}\left(\boldsymbol{t}-I_{2}\right)=\left(\begin{array}{rr}\omega^{0} & \omega^{+} \\ \omega^{-} & -\omega^{0}\end{array}\right), \quad \omega\left(x^{i}\right)=\omega_{H}\left(x^{i}\right)=\widetilde{\mathrm{d} x^{i}}$ for $i \in\{1,2,3\}$.

This extends to the whole of $\widetilde{A}^{+}$as a right $\widetilde{A}$-module map. To see that $\omega$ is well-defined, it suffices to check

$$
\omega\left(x^{i} \boldsymbol{t}-\boldsymbol{t} x^{i}\right)=\omega\left(\left[x^{i}, \boldsymbol{t}\right]\right) \quad \text { for all } i \in\{1,2,3\},
$$

where $\left[x^{i}, t\right]$ are cross relations (5-8) computed in Example 5.3. On the one hand,

$$
\begin{aligned}
\omega\left(x^{i} \boldsymbol{t}-\boldsymbol{t} x^{i}\right) & =\omega\left(x^{i} \boldsymbol{t}-\left(\boldsymbol{t}-I_{2}\right) x^{i}-x^{i} I_{2}\right) \\
& =\omega_{H}\left(x^{i}\right) \triangleleft \boldsymbol{t}-\omega_{A}\left(\boldsymbol{t}-I_{2}\right) \triangleleft x^{i}-\omega_{H}\left(x^{i}\right) I_{2} \\
& =-\omega_{A}\left(\boldsymbol{t}-I_{2}\right) \triangleleft x^{i},
\end{aligned}
$$

that is,

$$
\omega\left(x^{i} \boldsymbol{t}-\boldsymbol{t} x^{i}\right)=-\left(\begin{array}{rr}
\omega^{0} & \omega^{+} \\
\omega^{-} & -\omega^{0}
\end{array}\right) \triangleleft x^{i}
$$

Since

$$
\begin{aligned}
{\left[x^{1}, \boldsymbol{t}\right] } & =-\lambda b c t e_{2}+\frac{\lambda}{2} \boldsymbol{t} \operatorname{diag}(a c,-b d)+\frac{\lambda}{2} \operatorname{diag}(b,-c) \\
& =-\lambda b c t e_{2}+\frac{\lambda}{2}\left(t-I_{2}\right) \operatorname{diag}(a c,-b d)+\frac{\lambda}{2} \operatorname{diag}(c a,-b d)+\frac{\lambda}{2} \operatorname{diag}(b,-c),
\end{aligned}
$$


we know

$$
\begin{aligned}
\omega\left(\left[x^{1}, \boldsymbol{t}\right]\right)= & -\lambda \omega(b) \epsilon\left(c t e_{2}\right)+\frac{\lambda}{2} \omega\left(\left(\boldsymbol{t}-I_{2}\right)\right) \epsilon(\operatorname{diag}(a c,-b d)) \\
& +\frac{\lambda}{2} \operatorname{diag}(\omega(c) \triangleleft a,-\omega(b) \triangleleft d)+\frac{\lambda}{2} \operatorname{diag}(\omega(b),-\omega(c)) \\
= & \frac{\lambda}{2} \operatorname{diag}\left(\omega^{+}+\omega^{-},-\omega^{+}-\omega^{-}\right),
\end{aligned}
$$

using $\epsilon(\boldsymbol{t})=I_{2}$. Likewise, we have

$$
\begin{aligned}
& \omega\left(\left[x^{1}, \boldsymbol{t}\right]\right)=\frac{\lambda}{2}\left(\begin{array}{cc}
\omega^{+}+\omega^{-} & 0 \\
0 & -\omega^{+}-\omega^{-}
\end{array}\right), \\
& \omega\left(\left[x^{2}, \boldsymbol{t}\right]\right)=\frac{\imath \lambda}{2}\left(\begin{array}{cc}
\omega^{+}-\omega^{-} & 0 \\
0 & -\omega^{+}+\omega^{-}
\end{array}\right), \\
& \omega\left(\left[x^{3}, \boldsymbol{t}\right]\right)=\lambda\left(\begin{array}{cc}
0 & -\omega^{+} \\
-\omega^{-} & 0
\end{array}\right) .
\end{aligned}
$$

Comparing with (5-15), we see that $\omega\left(x^{i} \boldsymbol{t}-\boldsymbol{t} x^{i}\right)=\omega\left(\left[x^{i}, \boldsymbol{t}\right]\right)$ holds for each $i=1,2,3$ if and only if the right $H$-action on $\Lambda_{A}^{1}$ is the one defined by (5-14). From the coproduct of $x^{i}$ given in Example 5.3, we know

$$
\mathrm{d} x^{i}=\widetilde{\mathrm{d} x^{i}}+\frac{1}{2} x^{k} \omega\left(\pi_{\epsilon}\left(\operatorname{Tr}\left(\boldsymbol{t} \sigma_{i} \boldsymbol{t}^{-1} \sigma_{k}\right)\right)\right) .
$$

This gives rise to the formulae for derivatives on $x^{i}$ as displayed.

We now analyse when a Poisson-compatible left-covariant flat preconnection is bicovariant.

Lemma 5.9. Let $\mathfrak{g}$ be in the setting of Theorem 5.6. The pre-Lie structure o given by (5-12) of $\underline{\mathfrak{g}^{*}} \triangleleft \overline{\mathfrak{g}^{*}}$ gives a bicovariant preconnection in Corollary 4.2 if and only if

$$
\begin{gathered}
\delta_{\mathfrak{g}^{*}}(f \circ g)=0, \quad f_{(1)} \otimes\left[f_{(2)}, g\right]_{\mathfrak{g}^{*}}=0, \\
f_{(1)} \circ g \otimes f_{(2)}=-f \circ g_{(1)} \otimes g_{(2)}, \\
\delta_{\mathfrak{g}^{*}}(\phi * \psi)=0, \quad \phi * f_{(1)} \otimes f_{(2)}=0,
\end{gathered}
$$

for all $\phi, \psi \in \underline{\mathfrak{g}^{*}}, f, g \in \overline{\mathfrak{g}^{*}}$.

Proof. Since the bicovariance condition (4-6) is bilinear on entries, it suffices to show that o obeys (4-6) on any pair of elements $(\phi, \psi),(\phi, f),(f, \phi)$ and $(f, g)$ if and only if all the displayed identities hold for any $\phi, \psi \in \underline{\mathfrak{g}^{*}}, f, g \in \overline{\mathfrak{g}^{*}}$.

Firstly, for any $f \in \overline{\mathfrak{g}^{*}}$ and $\phi \in \underline{\mathfrak{g}^{*}}$, (4-6) on õ reduces to $\delta_{\mathfrak{g}^{*}}[f, \phi]_{\mathfrak{g}^{*}}-\left[f, \phi_{(1)}\right] \otimes \phi_{(2)}-\phi_{(1)} \otimes\left[f, \phi_{(2)}\right]=\underline{f_{(1)}} * \phi \otimes \overline{f_{(2)}}+\left[\overline{f_{(1)}}, \phi\right] \otimes f_{(2)}$. 
The only term in the above identity not lying in $\mathfrak{g}^{*} \otimes \mathfrak{g}^{*}$ is $f_{(1)} * \phi \otimes \overline{f_{(2)}}$, which hence equals zero. Noting that $\delta_{\mathfrak{g}^{*}}$ is a 1-cocycle, the remaining terms imply that $f_{(1)} \otimes\left[f_{(2)}, \phi\right]_{\mathfrak{g}^{*}}=0$. Changing the role of $f$ and $\phi$ in (4-6) implies $\phi * f_{(1)} \otimes \overline{f_{(2)}}=0$, as required.

Next, for any $f, g \in \overline{\mathfrak{g}^{*}}$, the condition (4-6) on õ requires

$$
\begin{aligned}
& \underline{(f \circ g)_{(1)}} \otimes \overline{(f \circ g)_{(2)}}+\overline{(f \circ g)_{(1)}} \otimes \underline{(f \circ g)_{(2)}}-\left[f, g_{(2)}\right]_{\mathfrak{g}^{*}} \otimes \overline{g_{(2)}} \\
& -f \circ \overline{g_{(1)}} \otimes \underline{g_{(2)}}-\underline{g_{(1)}} \otimes f \circ \overline{g_{(2)}}-\overline{g_{(1)}} \otimes\left[f, \underline{g_{(2)}}\right]_{\mathfrak{g}^{*}} \\
& =\left[\underline{f_{(1)}}, g\right]_{\mathfrak{g}^{*}} \otimes \overline{f_{(2)}}+\overline{f_{(1)}} \circ g \otimes \underline{f_{(2)}}-\underline{g_{(1)}} \otimes \overline{g_{(2)}} \circ f .
\end{aligned}
$$

The terms in the above identity lying in $\overline{\mathfrak{g}^{*}} \otimes \mathfrak{g}^{*}$ are exactly the condition (4-6) on the pre-Lie structure $\circ$ for $\overline{\mathfrak{g}^{*}}$. Cancelling this, the remaining terms in $\mathfrak{g}^{*} \otimes \overline{\mathfrak{g}^{*}}$ reduce to $g_{(1)} \circ f \otimes g_{(2)}+g \circ f_{(1)} \otimes f_{(2)}=0$, which is equivalent to

$$
f_{(1)} \circ g \otimes f_{(2)}+f \circ g_{(1)} \otimes g_{(2)}=0 \quad \text { for all } f, g \in \mathfrak{g}^{*} .
$$

Combining the above with $f_{(1)} \otimes\left[f_{(2)}, \phi\right]_{\mathfrak{g}^{*}}=0$, the condition (4-6) on $\circ$ reduces to $\delta_{\mathfrak{g}^{*}}(f \circ g)=0$.

Finally, for any $\phi, \psi \in \mathfrak{g}^{*}$, the condition (4-6) on õ reduces to (4-6) on $*$ for $\mathfrak{g}^{*}$. Since $*$ is commutative, this eventually becomes

$$
(\phi * \psi)_{(1)} \otimes(\phi * \psi)_{(2)}=\phi * \psi_{(1)} \otimes \psi_{(2)}+\phi_{(1)} * \psi \otimes \phi_{(2)} .
$$

Since $\phi * f_{(1)} \otimes f_{(2)}=0$, this reduces to $\delta_{\mathfrak{g}^{*}}(\phi * \psi)=0$.

The conditions in Lemma 5.9 all hold when the Lie bracket of $\mathfrak{g}$ (or the Lie cobracket of $\mathfrak{g}^{*}$ ) vanishes. Putting these results together we have:

Proposition 5.10. Let $G$ be a finite-dimensional connected and simply connected Poisson-Lie group with Lie bialgebra $\mathfrak{g}$. Assume that $\left(\mathfrak{g}^{*},[,]_{\mathfrak{g}^{*}}\right)$ obeys the conditions in Theorem 5.6 and Lemma 5.9. Then the tangent bundle $\bar{G} \bowtie \underline{\mathfrak{g}}$ in Lemma 5.1 admits a Poisson-compatible bicovariant flat preconnection.

Example 5.11. In the setting of Example 5.2, we already know from Corollary 4.2 that the abelian Poisson-Lie group $\mathbb{R}^{n}>\mathfrak{m}^{*}$ admits a Poisson-compatible leftcovariant (bicovariant) flat preconnection if and only if $\left(\overline{\mathfrak{m}^{*}} \succ \mathfrak{m}^{*}\right)^{*}=\underline{\mathfrak{m}} \triangleleft_{\text {ad }} \mathfrak{m}$ admits a pre-Lie structure.

From Corollary 5.5, we know that such a pre-Lie structure o exists and is given by $(x, \xi) \tilde{o}(y, \eta)=\left(x \cdot y+[\xi, y]_{\mathfrak{m}}, \xi \circ \eta\right)$ if we assume $\left(\mathfrak{m}, \cdot,[,]_{\mathfrak{m}}\right)$ to be a finitedimensional (not necessarily unital) Poisson algebra such that $\left(\mathfrak{m},[,]_{\mathfrak{m}}\right)$ admits a pre-Lie structure $\circ: \mathfrak{m} \otimes \mathfrak{m} \rightarrow \mathfrak{m}$. Then the corresponding preconnection is

$$
\gamma((x, \xi), \mathrm{d}(y, \eta))=\mathrm{d}\left(x \cdot y+[\xi, y]_{\mathfrak{m}}, \xi \circ \eta\right)
$$

for any $x, y \in \underline{\mathfrak{m}}, \xi, \eta \in \mathfrak{m}$. 
In fact this extends to all orders. Under the assumptions above, according to Proposition 4.4, the noncommutative algebra $U_{\lambda}\left(\underline{\mathfrak{m}} \triangleleft_{\text {ad }} \mathfrak{m}\right)=S(\underline{\mathfrak{m}}) \rtimes U_{\lambda}(\mathfrak{m})$, or the cross product of algebras $\mathbb{C}\left[\mathbb{R}^{n}\right] \rtimes U(\mathfrak{m})$ (as quantisation of $C^{\infty}\left(\mathbb{R}^{n}>\mathfrak{m}^{*}\right)$ ), admits a connected bicovariant differential graded algebra

$$
\Omega\left(U_{\lambda}\left(\underline{\mathfrak{m}} \rtimes_{\mathrm{ad}} \mathfrak{m}\right)\right)=\left(S(\underline{\mathfrak{m}}) \rtimes U_{\lambda}(\mathfrak{m})\right) \bowtie \Lambda\left(\underline{\mathfrak{m}} \rtimes_{\mathrm{ad}} \mathfrak{m}\right)
$$

as quantisation. Note that $\mathrm{d}(x, \xi)=1 \otimes(x, \xi) \in 1 \otimes \Lambda^{1}$. The commutation relations on generators are

$$
\begin{gathered}
{[\xi, \eta]=\lambda[\xi, \eta]_{\mathfrak{m}}, \quad[x, y]=0, \quad[\xi, x]=\lambda[\xi, x]_{\mathfrak{m}},} \\
{[x, \mathrm{~d} y]=\lambda \mathrm{d}(x \cdot y), \quad[\xi, \mathrm{d} x]=\lambda \mathrm{d}[\xi, x]_{\mathfrak{m}}, \quad[\xi, \mathrm{d} \eta]=\lambda \mathrm{d}(\xi \circ \eta),}
\end{gathered}
$$

for any $x, y \in \underline{\mathfrak{m}}, \xi, \eta \in \mathfrak{m}$.

\section{Semiclassical data on the cotangent bundle $T^{*} G=\mathfrak{g}^{*}>\triangleleft G$}

In this section, we focus on the semiclassical data for quantisation of the cotangent bundle $T^{*} G$ of a Poisson-Lie group $G$. We aim to construct preconnections on $T^{*} G$.

As a Lie group, the cotangent bundle $T^{*} G$ can be identified with the semidirect product of Lie groups $\mathfrak{g}^{*} \rtimes G$ with product given by

$$
(\phi, g)(\psi, h)=\left(\phi+\operatorname{Ad}^{*}(g)(\psi), g h\right)
$$

for any $g, h \in G, \phi, \psi \in \mathfrak{g}^{*}$. As before, $\mathfrak{g}^{*}$ is $\mathfrak{g}^{*}$ but viewed as an abelian Lie group under addition. In particular,

$$
(\phi, g)^{-1}=\left(-\operatorname{Ad}^{*}\left(g^{-1}\right)(\phi), g^{-1}\right) \quad \text { and } \quad(0, g)(\phi, e)(0, g)^{-1}=\left(\operatorname{Ad}^{*}(g) \phi, e\right) .
$$

Here $\mathrm{Ad}^{*}$ is the coadjoint action of $G$ on the dual of its Lie algebra. The Lie algebra of $T^{*} G$ is then identified with the semidirect sum of Lie algebras $\underline{\mathfrak{g}}^{*} \rtimes \mathfrak{g}$, where the Lie bracket of $\mathfrak{g}^{*} \triangleleft \mathfrak{g}$ is given by

$$
[(\phi, x),(\psi, y)]=\left(\operatorname{ad}_{x}^{*} \psi-\operatorname{ad}_{y}^{*} \phi,[x, y]_{\mathfrak{g}}\right)
$$

for any $\phi, \psi \in \mathfrak{g}^{*}, x, y \in \mathfrak{g}$. Here $\mathfrak{g}^{*}$ is $\mathfrak{g}^{*}$ viewed as abelian Lie algebra and ad* denotes the usual left coadjoint action of $\mathfrak{g}$ on $\mathfrak{g}^{*}$ (or $\mathfrak{g}^{*}$ ).

Our strategy to build Poisson-Lie structures on the cotangent bundle here is to construct Lie bialgebra structures on $\mathfrak{g}^{*} \rtimes \mathfrak{g}$ via bosonisation of Lie bialgebras. Then we can exponentiate the obtained Lie cobracket of $\mathfrak{g}^{*} \rtimes \mathfrak{g}$ to a Poisson-Lie structure on $\mathfrak{g}^{*} \triangleleft G$. We can always do this, as we work in the nice case where the Lie group is connected and simply connected. 
6A. Lie bialgebra structures on $\mathfrak{g}^{*}>\triangleleft \mathfrak{g}$ via bosonisation. Let $\mathfrak{g} \mathcal{M}$ denote the monoidal category of left Lie $\mathfrak{g}$-crossed modules. A braided-Lie bialgebra $\mathfrak{b} \in \mathfrak{g} \mathcal{\mathfrak { g }} M$ is $\left(\mathfrak{b},[,]_{\mathfrak{b}}, \delta_{\mathfrak{b}}, \triangleright, \beta\right)$ given by a $\mathfrak{g}$-crossed module $(\mathfrak{b}, \triangleright, \beta)$ that is both a Lie algebra $\left(\mathfrak{b},[,]_{\mathfrak{b}}\right)$ and a Lie coalgebra $\left(\mathfrak{b}, \delta_{\mathfrak{b}}\right)$ living in $\mathfrak{g}_{\mathfrak{g}} \mathcal{M}$, with the infinitesimal braiding $\Psi: \mathfrak{b} \otimes \mathfrak{b} \rightarrow \mathfrak{b} \otimes \mathfrak{b}$ obeying $\Psi(x, y)=\operatorname{ad}_{x} \delta_{\mathfrak{b}} y-\operatorname{ad}_{y} \delta_{\mathfrak{b}} x-\delta_{\mathfrak{b}}\left([x, y]_{\mathfrak{b}}\right)$ for any $x, y \in \mathfrak{b}$. If $\mathfrak{b}$ is a braided-Lie bialgebra in ${ }_{\mathfrak{g}}^{\mathfrak{g}} \mathcal{M}$, then the bisum $\mathfrak{b} \rtimes \mathfrak{g}$ with semidirect Lie bracket/cobracket is a Lie bialgebra [Majid 2000].

For our purposes, a straightforward solution is to ask for

$$
\underline{\mathfrak{g}^{*}}=\left(\mathfrak{g}^{*},[,]=0, \delta_{\mathfrak{g}^{*}}, \mathrm{ad}^{*}, \alpha\right)
$$

to be a braided-Lie algebra in ${ }_{\mathfrak{g}}^{\mathfrak{g}} \mathcal{M}$ for some left $\mathfrak{g}$-coaction $\alpha$ on $\mathfrak{g}^{*}$.

Lemma 6.1. Let $\mathfrak{g}$ be a finite-dimensional Lie bialgebra and suppose there is a linear map $\Xi: \mathfrak{g}^{*} \otimes \mathfrak{g}^{*} \rightarrow \mathfrak{g}^{*}$ such that (2-6) holds. Then

$$
\underline{\mathfrak{g}^{*}}=\left(\mathfrak{g}^{*},[,]=0, \delta_{\mathfrak{g}^{*}}, \mathrm{ad}^{*}, \alpha\right)
$$

is a braided-Lie bialgebra in $\mathfrak{g}_{\mathfrak{g}}^{\mathfrak{M}}$ if and only if $\Xi$ is a pre-Lie structure on $\mathfrak{g}^{*}$ such that $\Xi$ is covariant under the Lie cobracket $\delta_{\mathfrak{g}^{*}}$, in the sense that

$$
\Xi(\phi, \psi)_{(1)} \otimes \Xi(\phi, \psi)_{(2)}=\Xi\left(\phi, \psi_{(1)}\right) \otimes \psi_{(2)}+\psi_{(1)} \otimes \Xi\left(\phi, \psi_{(2)}\right)
$$

and

$$
\Xi\left(\phi_{(1)}, \psi\right) \otimes \phi_{(2)}=\psi_{(1)} \otimes \Xi\left(\psi_{(2)}, \phi\right)
$$

for any $\phi, \psi \in \mathfrak{g}^{*}$. Here the left $\mathfrak{g}$-coaction $\alpha$ and the left pre-Lie product $\Xi$ of $\mathfrak{g}^{*}$ are mutually determined via

$$
\langle\alpha(\phi), \psi \otimes x\rangle=-\Xi(\psi, \phi)(x)
$$

for any $\phi, \psi \in \mathfrak{g}^{*}, x \in \mathfrak{g}$. In this case, the bisum $\mathfrak{g}^{*} \rtimes \mathfrak{g}$ is a Lie bialgebra with Lie bracket given by (6-1) and Lie cobracket given by

$$
\delta(\phi, X)=\delta_{\mathfrak{g}} X+\delta_{\mathfrak{g}^{*}} \phi+(\mathrm{id}-\tau) \alpha(\phi)
$$

for any $\phi \in \mathfrak{g}^{*}, X \in \mathfrak{g}$.

Proof. Since the Lie bracket is zero, by definition, the question amounts to finding a left $\mathfrak{g}$-coaction $\alpha$ on $\mathfrak{g}^{*}$ such that (1) ( $\left.\mathrm{ad}^{*}, \alpha\right)$ makes $\mathfrak{g}^{*}$ into a left $\mathfrak{g}$-crossed module; (2) $\delta_{\mathfrak{g}^{*}}$ is a left $\mathfrak{g}$-comodule map under $\alpha$; and (3) the infinitesimal braiding $\Psi$ on $\underline{\mathfrak{g}^{*}}$ is trivial, i.e.,

(6-6) $\Psi(\phi, \psi)=\mathrm{ad}_{\psi^{(1)}}^{*} \phi \otimes \psi^{(2)}-\mathrm{ad}_{\phi^{(1)}}^{*} \psi \otimes \phi^{(2)}-\psi^{(2)} \otimes \mathrm{ad}_{\psi^{(1)}}^{*} \phi+\phi^{(2)} \otimes \mathrm{ad}_{\phi^{(1)}}^{*} \psi$ is zero for any $\phi, \psi \in \underline{\mathfrak{g}}^{*}$, where we write $\alpha(\phi)=\phi^{(1)} \otimes \phi^{(2)}$. 
Clearly, $\alpha$ is a left $\mathfrak{g}$-coaction on $\mathfrak{g}^{*}$ if and only if $\Xi$ defines a left $\mathfrak{g}^{*}$ action on itself, since $\alpha$ and $\Xi$ are adjoint to each other by (6-4), thus if and only if $\Xi$ is left pre-Lie structure, due to (2-6). Next, the condition that the Lie cobracket $\delta_{\mathfrak{g}^{*}}$ is a left $\mathfrak{g}$-comodule map under $\alpha$ means $\delta_{\mathfrak{g}^{*}}$ is a right $\mathfrak{g}^{*}$-module map under $-\Xi$. This is exactly the assumption (6-2) on $\Xi$. In this case, the cross condition (3-2) or (4-6) (using compatibility) for making $\mathfrak{g}^{*}$ a left $\mathfrak{g}$-crossed module under ( $\mathrm{ad}^{*}, \alpha$ ) becomes (6-3).

It suffices to show that the infinitesimal braiding $\Psi$ on $\mathfrak{g}^{*}$ is trivial on $\mathfrak{g}^{*}$. By construction,

$$
\langle\alpha(\phi), \varphi \otimes x\rangle=-\Xi(\varphi, \phi)(x),
$$

so

$$
\operatorname{ad}_{\psi^{(1)}}^{*} \phi \otimes \psi^{(2)}=\phi_{(2)} \otimes \Xi\left(\phi_{(1)}, \psi\right),
$$

where

$$
\alpha(\phi)=\phi^{(1)} \otimes \phi^{(2)} \quad \text { and } \quad \delta_{\mathfrak{g}^{*}} \phi=\phi_{(1)} \otimes \phi_{(2)} .
$$

Thus, using (6-3),

$$
\begin{gathered}
\Psi(\phi, \psi)=\operatorname{ad}_{\psi^{(1)}}^{*} \phi \otimes \psi^{(2)}-\mathrm{ad}_{\phi^{(1)}}^{*} \psi \otimes \phi^{(2)}-\psi^{(2)} \otimes \mathrm{ad}_{\psi^{(1)}}^{*} \phi+\phi^{(2)} \otimes \mathrm{ad}_{\phi^{(1)}}^{*} \psi \\
=\Xi\left(\psi_{(1)}, \phi\right) \otimes \psi_{(2)}-\psi_{(2)} \otimes \Xi\left(\psi_{(1)}, \phi\right) \\
-\Xi\left(\phi_{(1)}, \psi\right) \otimes \phi_{(2)}+\phi_{(2)} \otimes \Xi\left(\phi_{(1)}, \psi\right) \\
=\Xi\left(\psi_{(1)}, \phi\right) \otimes \psi_{(2)}+\psi_{(1)} \otimes \Xi\left(\psi_{(2)}, \phi\right) \\
-\Xi\left(\phi_{(1)}, \psi\right) \otimes \phi_{(2)}-\phi_{(1)} \otimes \Xi\left(\phi_{(2)}, \psi\right) \\
=0 .
\end{gathered}
$$

Example 6.2. Let $\mathfrak{m}$ be a pre-Lie algebra with product $\circ: \mathfrak{m} \otimes \mathfrak{m} \rightarrow \mathfrak{m}$ and $\mathfrak{g}=\mathfrak{m}^{*}$ with zero Lie bracket as in Example 4.3. This meets the conditions in Lemma 6.1 and we have a Lie bialgebra $\mathfrak{g}^{*}>\mathfrak{g}=\underline{\mathfrak{m}}>\mathfrak{m}^{*}$ with zero Lie bracket and with Lie cobracket

$$
\delta \phi=\delta_{\mathfrak{m}^{*}} \phi \quad \text { and } \quad \delta x=(\mathrm{id}-\tau) \alpha(x) \quad \text { for all } \phi \in \mathfrak{m}^{*}, x \in \mathfrak{m},
$$

where $\alpha$ is given by the pre-Lie algebra structure $\circ$ on $\mathfrak{m}$, i.e., $\langle x \otimes \phi, \alpha(y)\rangle=$ $-\langle\phi, x \circ y\rangle$. The Lie bialgebra here is the dual of the semidirect sum Lie algebra $\tilde{\mathfrak{m}}=\mathfrak{m}^{*} \rtimes \mathfrak{m}$ (viewed as a Lie bialgebra with zero Lie cobracket), where $\mathfrak{m}$ acts on $\mathfrak{m}^{*}$ by the adjoint to the action of $\mathfrak{m}$ on $\mathfrak{m}$ given by o, i.e., $\langle x \triangleright \phi, y\rangle=-\phi(x \circ y)$, $[x, y]=[x, y]_{\mathfrak{m}}, \quad[x, \phi]=x \triangleright \phi \quad$ and $\quad[\phi, \psi]=0 \quad$ for all $x, y \in \mathfrak{m}, \phi, \psi \in \mathfrak{m}^{*}$. The Poisson bracket on $\tilde{\mathfrak{m}}^{*}=\underline{\mathfrak{m}}>\mathfrak{m}^{*}$ is then the Kirillov-Kostant one for $\tilde{\mathfrak{m}}$, i.e., given by this Lie bracket. 
Example 6.3. Let $\mathfrak{g}$ be a quasitriangular Lie bialgebra with $r$-matrix

$$
r=r^{(1)} \otimes r^{(2)} \in \mathfrak{g} \otimes \mathfrak{g}
$$

such that $r_{+} \triangleright X=0$ for all $X \in \mathfrak{g}$. As in Example 4.7, $\mathfrak{g}^{*}$ is a pre-Lie algebra with product $\Xi(\phi, \psi)=-\left\langle\phi, r^{(2)}\right\rangle \operatorname{ad}_{r^{(1)}}^{*} \psi$. Direct computation shows $\Xi$ satisfies (6-2)-(6-3) without any further requirement. So $\mathfrak{g}^{*}=\left(\mathfrak{g}^{*},[]=0,, \delta_{\mathfrak{g}^{*}}, \mathrm{ad}^{*}, \alpha\right)$ is a braided-Lie bialgebra in $\mathfrak{g}_{\mathfrak{g}}^{\mathfrak{g}} \mathcal{\text { with }} \alpha(\phi)=r^{(2)} \otimes \mathrm{ad}_{r^{(1)}}^{*} \phi$. Hence, from Lemma 6.1, $\mathfrak{g}^{*} \rtimes \mathfrak{g}$ is a Lie bialgebra with Lie bracket given by (6-1) and Lie cobracket given by (6-5), i.e.,

$$
\delta(\phi, X)=\delta_{\mathfrak{g}} X+\delta_{\mathfrak{g}^{*}} \phi+(\mathrm{id}-\tau)\left(r^{(2)} \otimes \operatorname{ad}_{r^{(1)}}^{*} \phi\right) .
$$

Note that if $\mathfrak{g}$ is a quasitriangular Lie bialgebra, Majid [2000, Corollary 3.2, Lemma 3.4] shows that $\left(\mathfrak{g}^{*}, \delta_{\mathfrak{g}^{*}}\right)$ is a braided-Lie bialgebra with Lie bracket given by

$$
[\phi, \psi]=2\left\langle\phi, r_{+}^{(1)}\right\rangle \operatorname{ad}_{r_{+}^{(2)}}^{*} \psi=0
$$

in our case, so in this example $\mathfrak{g}^{*}$ in Lemma 6.1 agrees with a canonical construction. On the other hand, this class of examples is more useful in the case where $\mathfrak{g}$ is triangular.

6B. Poisson-Lie structures on $\underline{\mathfrak{g}}^{*} \rtimes \boldsymbol{G}$ induced from $\mathfrak{g}^{*} \rtimes \mathfrak{g}$. Next we exponentiate our Lie bialgebra structure $\overline{\mathfrak{g}^{*}} \triangleleft \mathfrak{g}$ constructed by Lemma 6.1 to a Poisson-Lie structure on the cotangent bundle. As usual this is done by exponentiating $\delta$ to a group 1-cocycle $D$.

Proposition 6.4. Let $G$ be a connected and simply connected Poisson-Lie group. If its Lie algebra $\mathfrak{g}$ with a given coaction $\alpha$ meets the conditions of Lemma 6.1 then $\underline{\mathfrak{g}^{*}} \triangleleft G$ is a Poisson-Lie group with

$$
D(\phi, g)=\operatorname{Ad}_{\phi} D(g)+\delta_{\mathfrak{g}^{*}} \phi+(\mathrm{id}-\tau)\left(\phi^{(1)} \otimes \phi^{(2)}-\frac{1}{2} \mathrm{ad}_{\phi^{(1)}}^{*} \phi \otimes \phi^{(2)}\right),
$$

where $\alpha(\phi)=\phi^{(1)} \otimes \phi^{(2)}$.

Proof. Because of the cocycle condition, it suffices to find $D(\phi):=D(\phi, e)$ and $D(g):=D(e, g)$; then

$$
D(\phi, g)=D(\phi)+\operatorname{Ad}_{\phi} D(g) \text { for all }(\phi, g) \in \mathfrak{g}^{*} \rtimes G,
$$

where

$$
\operatorname{Ad}_{\phi}(X)=X-\operatorname{ad}_{X}^{*} \phi \quad \text { for all } X \in \mathfrak{g} \subset \mathfrak{g}^{*} \rtimes \mathfrak{g}, \phi \in \mathfrak{g}^{*}
$$

We require

$$
\frac{\mathrm{d}}{\mathrm{d} t} D(t \phi)=\operatorname{Ad}_{t \phi}(\delta \phi)
$$


which we solve writing

$$
D(\phi)=\delta_{\mathfrak{g}^{*}} \phi+Z(\phi),
$$

so that

$$
\begin{aligned}
\frac{\mathrm{d}}{\mathrm{d} t} Z(t \phi) & =\operatorname{Ad}_{t \phi}((\mathrm{id}-\tau) \circ \alpha(\phi))=(\mathrm{id}-\tau) \circ \alpha(\phi)-t(\mathrm{id}-\tau)\left(\mathrm{ad}_{\phi^{(1)}}^{*} \phi \otimes \phi^{(2)}\right), \\
Z(0) & =0 .
\end{aligned}
$$

Integrating this to

$$
Z(t \phi)=t(\mathrm{id}-\tau) \circ \alpha(\phi)-\frac{1}{2} t^{2}(\mathrm{id}-\tau)\left(\mathrm{ad}_{\phi^{(1)}}^{*} \phi \otimes \phi^{(2)}\right),
$$

we obtain

$$
D(\phi)=\delta_{\mathfrak{g}^{*}} \phi+(\mathrm{id}-\tau)\left(\phi^{(1)} \otimes \phi^{(2)}-\frac{1}{2} \mathrm{ad}_{\phi^{(1)}}^{*} \phi \otimes \phi^{(2)}\right),
$$

where $\alpha(\phi)=\phi^{(1)} \otimes \phi^{(2)}$. The general case $\mathrm{d} D(\phi+t \psi) /\left.\mathrm{d} t\right|_{t=0}=\operatorname{Ad}_{\phi}(\delta \psi)$ amounts to the vanishing of the expression (6-6), which we saw holds under our assumptions in the proof of Lemma 6.1.

Example 6.5. In the setting of Example 6.3 with $(\mathfrak{g}, r)$ quasitriangular such that $r_{+} \triangleright X=0$ for all $X \in \mathfrak{g}$, we know that $\mathfrak{g}^{*} \rtimes G$ is a Poisson-Lie group with

$$
D(\phi, g)=\delta_{\mathfrak{g}^{*}} \phi+\operatorname{Ad}_{(\phi, g)}(r)-r+2 r_{+} \triangleright \phi-r_{+} \triangleright(\phi \otimes \phi),
$$

where $\triangleright$ denotes the coadjoint action ad*. As $\alpha(\phi)=r_{21} \triangleright \phi$, direct computation shows that $D(\phi)=\delta_{\mathfrak{g}^{*}}(\phi)+(\mathrm{id}-\tau) r_{21} \triangleright \phi+r_{-} \triangleright(\phi \otimes \phi)$. Since the differential equation for $D(g)$ is the usual one on $G$ for $\mathfrak{g}$ quasitriangular, $D(g)=\operatorname{Ad}_{g}(r)-r$ and we obtain the stated result. Note that

$$
\begin{aligned}
\operatorname{Ad}_{\phi}(r) & =\left(r^{(1)}-r^{(1)} \triangleright \phi\right) \otimes\left(r^{(2)}-r^{(2)} \triangleright \phi\right) \\
& =r+r \triangleright(\phi \otimes \phi)-r^{(1)} \triangleright \phi \otimes r^{(2)}-r^{(1)} \otimes r^{(2)} \triangleright \phi .
\end{aligned}
$$

The differential equation $\mathrm{d} D(\phi+t \psi) /\left.\mathrm{d} t\right|_{t=0}=\operatorname{Ad}_{\phi}(\delta \psi)$ amounts to

$$
r_{+} \triangleright(\mathrm{id}-\tau)(\phi \otimes \psi)=0,
$$

which is guaranteed by $r_{+} \triangleright X=0$ for all $X \in \mathfrak{g}$.

Note that we can view $r \in\left(\mathfrak{g}^{*} \rtimes \mathfrak{g}\right)^{\otimes 2}$, where it will obey the the classical Yang-Baxter equation and, in our case, $\operatorname{ad}_{\phi}\left(r_{+}\right)=0$ as $r_{+} \triangleright \phi=0$ on $\mathfrak{g}^{*}$ under our assumptions. In this case $\underline{\mathfrak{g}}^{*} \rtimes \mathfrak{g}$ is quasitriangular with the same $r$, with Lie cobracket

$$
\delta_{r}(\phi)=\operatorname{ad}_{\phi}(r)=-r^{(1)} \triangleright \phi \otimes r^{(2)}-r^{(1)} \otimes r^{(2)} \triangleright \phi=(\mathrm{id}-\tau) r_{21} \triangleright \phi
$$

at the Lie algebra level (differentiating the above $\operatorname{Ad}_{t \phi}$ ) and with $\delta X$ as before. In our case the cobracket has an additional $\delta_{\mathfrak{g}^{*}} \phi$ term reflected also in $D$. 
6C. Preconnections on the cotangent bundle $\mathfrak{g}^{*} \rtimes G$. Let $\mathfrak{g}$ be a finite-dimensional Lie bialgebra and suppose that its dual $\mathfrak{g}^{*}$ admits a pre-Lie structure

$$
\Xi: \mathfrak{g}^{*} \otimes \mathfrak{g}^{*} \rightarrow \mathfrak{g}^{*}
$$

such that (6-2) and (6-3) hold as in the setting of Lemma 6.1. Then the dual of the Lie bialgebra $\mathfrak{g}^{*} \rtimes \mathfrak{g}$ is $\overline{\mathfrak{g}} \rtimes \mathfrak{g}^{*}$, with Lie bracket the semidirect sum $\mathfrak{g} \rtimes \mathfrak{g}^{*}$ and Lie cobracket the semidirect cobracket $\overline{\mathfrak{g}}>\mathfrak{g}^{*}$, that is,

$$
\begin{gathered}
{[x, y]=[x, y]_{\mathfrak{g}}, \quad[\phi, x]=\phi \triangleright x, \quad[\phi, \psi]=[\phi, \psi]_{\mathfrak{g}^{*}},} \\
\delta x=(\mathrm{id}-\tau) \beta(x), \quad \delta \phi=\delta_{\mathfrak{g}^{*}} \phi,
\end{gathered}
$$

for any $x, y \in \mathfrak{g}, \phi, \psi \in \mathfrak{g}^{*}$. Here the left action and coaction of $\mathfrak{g}^{*}$ on $\mathfrak{g}$ are given by

$$
\langle\phi \triangleright x, \psi\rangle=-\Xi(\phi, \psi)(x) \text { and }\langle\beta(x), y \otimes \phi\rangle=\langle\phi,[x, y]\rangle,
$$

respectively.

Here again, we use Lemma 5.4 to construct pre-Lie algebra structures on the semidirect sum $\overline{\mathfrak{g}} \rtimes \mathfrak{g}^{*}$.

Theorem 6.6. Let $G$ be a connected and simply connected Poisson-Lie group with Lie bialgebra $\mathfrak{g}$. Let $\mathfrak{g}^{*}$ admit two pre-Lie structures $\Xi$ and $\circ$, with $\Xi$ obeying (6-2) and (6-3) as in the setting of Lemma 6.1. Let $\mathfrak{g}$ also admit a pre-Lie structure $*$ such that

$$
\phi \triangleright(x * y)=(\phi \triangleright x) * y+x *(\phi \triangleright y),
$$

for all $x, y \in \mathfrak{g}, \phi \in \mathfrak{g}^{*}$, where $\triangleright$ is defined by (6-8). Then the Lie algebra $\overline{\mathfrak{g}} \rtimes \mathfrak{g}^{*}$ admits a pre-Lie structure õ:

$$
(x, \phi) \tilde{\circ}(y, \psi)=(x * y+\phi \triangleright y, \phi \circ \psi),
$$

and the cotangent bundle $\mathfrak{g}^{*} \rtimes G$ admits a Poisson-compatible left-covariant flat preconnection.

Proof. Since $(\mathfrak{g}, \Xi)$ is in the setting of Lemma 6.1, the left $\mathfrak{g}^{*}$-action in the semidirect sum $\overline{\mathfrak{g}} \rtimes \mathfrak{g}^{*}$ is the one defined in (6-8). The rest is immediate from Lemma 5.4 and Corollary 4.2.

To construct a bicovariant preconnection, the pre-Lie structure constructed in Theorem 6.6 must satisfy the bicovariance condition (4-6).

Proposition 6.7. In the setting of Theorem 6.6, the pre-Lie structure õ of $\overline{\mathfrak{g}} \rtimes \mathfrak{g}^{*}$ defined by (6-10) obeys the bicovariance condition if and only if o obeys (4-6), * is 
associative and

$$
\begin{gathered}
{[x, y] * z=[y, z] * x,} \\
\left(\left(\operatorname{ad}_{x}^{*} \psi\right) \circ \phi\right)(y)+\Xi\left(\operatorname{ad}_{y}^{*} \phi, \psi\right)(x)=0, \\
\Xi(\phi, \psi)\left([x, y]_{\mathfrak{g}}\right)=\Xi\left(\phi, \operatorname{ad}_{y}^{*} \psi\right)(x)-\left(\phi \circ \operatorname{ad}_{x}^{*} \psi\right)(y),
\end{gathered}
$$

for any $x, y, z \in \mathfrak{g}$ and $\phi, \psi \in \mathfrak{g}^{*}$. The associated preconnection is then bicovariant.

Proof. Since (4-6) is bilinear, it suffices to show that (4-6) holds on any pair of elements $(x, y),(x, \phi),(\phi, x)$ and $(\phi, \psi)$ if and only if all the conditions and displayed identities hold. Here we write $\beta(x)=x^{1} \otimes x_{2} \in \mathfrak{g}^{*} \otimes \mathfrak{g}$, so we know

$$
\left\langle x^{1}, y\right\rangle x_{2}=[x, y]_{\mathfrak{g}}, \quad x^{1}\left\langle x_{2}, \phi\right\rangle=-\mathrm{ad}_{x}^{*} \phi .
$$

Firstly, for any $\phi, \psi \in \mathfrak{g}^{*}$, the condition (4-6) for õ reduces to (4-6) on the pre-Lie structure $\circ$ for $\mathfrak{g}^{*}$.

Secondly, for any $x, y \in \overline{\mathfrak{g}}$, the condition (4-6) requires

$$
\begin{array}{r}
(x * y)^{1} \otimes(x * y)_{2}-(x * y)_{2} \otimes(x * y)^{1}-x^{1} \triangleright y \otimes x_{2}+x_{2} * y \otimes x^{1}+x * y_{2} \otimes y^{1} \\
=y^{1} \otimes\left[x, y_{2}\right]_{\mathfrak{g}}+y_{2} \otimes y^{1} \triangleright x .
\end{array}
$$

The terms lying in $\overline{\mathfrak{g}} \otimes \overline{\mathfrak{g}}$ on both sides should be equal, i.e., $-x^{1} \triangleright y \otimes x_{2}=y_{2} \otimes y^{1} \triangleright x$, which is equivalent to $-\Xi\left(\operatorname{ad}_{x}^{*} \psi, \phi\right)(y)=\Xi\left(\operatorname{ad}_{y}^{*} \phi, \psi\right)(x)$. This is true from our assumption (6-3) on $\Xi$. The terms in $\overline{\mathfrak{g}} \otimes \mathfrak{g}^{*}$ give $[x * y, z]=[x, z] * y+x *[y, z]$, i.e., $*$ is associative. The terms in $\mathfrak{g}^{*} \otimes \overline{\mathfrak{g}}$ give $(x * y)^{1} \otimes(x * y)_{2}=y^{1} \otimes\left[x, y_{2}\right]_{\mathfrak{g}}$ and, applying the first factor to $z \in \mathfrak{g}$, we obtain $[x * y, z]=[x,[y, z]]$, which is equivalent to $[x, z] * y=[z, y] * x$.

Now, for any $x \in \mathfrak{g}, \phi \in \mathfrak{g}^{*}$, the condition (4-6) reduces to

$$
0=x^{1} \circ \phi \otimes x_{2}-\phi_{(1)} \otimes \phi_{(2)} \triangleright x .
$$

Applying $y \otimes \psi$, this becomes $-\Xi\left(\operatorname{ad}_{y}^{*} \phi, \psi\right)(x)=\left(\left(\operatorname{ad}_{x}^{*} \psi\right) \circ \phi\right)(y)$.

Finally, for any $\phi \in \mathfrak{g}^{*}, x \in \overline{\mathfrak{g}}$, the condition (4-6) requires

$$
\begin{aligned}
(\phi \triangleright x)^{1} & \otimes(\phi \triangleright x)_{2}-(\phi \triangleright x)_{2} \otimes(\phi \triangleright x)^{1}-\phi \circ x^{1} \otimes x_{2} \\
& +\phi \triangleright x_{2} \otimes x^{1}-x^{1} \otimes \phi \triangleright x_{2}+x_{2} \otimes \phi \circ x^{1}=\phi_{(1)} \triangleright x \otimes \phi_{(2)}+x_{2} \otimes x^{1} \circ \phi .
\end{aligned}
$$

The terms lying in $\mathfrak{g}^{*} \otimes \overline{\mathfrak{g}}$ give

$$
(\phi \triangleright x)^{1} \otimes(\phi \triangleright x)_{2}-\phi \circ x^{1} \otimes x_{2}-x^{1} \otimes \phi \triangleright x_{2}=0 .
$$

Applying $y \otimes \psi$, this is equivalent to

$$
-\Xi\left(\phi, \operatorname{ad}_{y}^{*} \psi\right)(x)+\left(\phi \circ \operatorname{ad}_{x}^{*} \psi\right)(y)+\Xi(\phi, \psi)\left([x, y]_{\mathfrak{g}}\right)=0 .
$$


Applying $\psi \otimes y$ to the terms lying in $\overline{\mathfrak{g}} \otimes \mathfrak{g}^{*}$, after cancelling the identity just obtained, we have $\left(\left(\operatorname{ad}_{x}^{*} \psi\right) \circ \phi\right)(y)+\Xi\left(\operatorname{ad}_{y}^{*} \phi, \psi\right)(x)=0$.

For simplicity, one can choose $\Xi=\circ$ in Theorem 6.6 and Proposition 6.7:

Corollary 6.8. Let $\mathfrak{g}$ be a finite-dimensional Lie bialgebra. Assume that $\mathfrak{g}^{*}$ admits a pre-Lie structure $\Xi$ such that (6-2) and (6-3) hold. Also assume that $\mathfrak{g}$ admits a pre-Lie structure * such that (6-9) holds, where the action is defined by (6-8) from $\Xi$. Then

$$
(x, \phi) \tilde{\circ}(y, \psi)=(x * y+\phi \triangleright y, \Xi(\phi, \psi))
$$

defines a pre-Lie structure for the Lie algebra $\overline{\mathfrak{g}} \rtimes \mathfrak{g}^{*}$, and thus provides a Poissoncompatible left-covariant flat preconnection on the cotangent bundle $\mathfrak{g}^{*} \rtimes G$. Moreover, if $*$ is associative and obeys (6-11), then the pre-Lie structure o obeys (4-6) and the corresponding preconnection is bicovariant.

Proof. Clearly, there is no further condition on $\circ$ in the case $\circ=\Xi$ in Theorem 6.6. In the bicovariant case, the further conditions on $\circ$ in Proposition 6.7 are (4-6), (6-12) and (6-13). These all can be proven from the assumptions (6-2) and (6-3) we already made on $\Xi$. In particular, (6-3) shows that (6-12) is true, and (6-2) is simply a variation of (6-13) when $\circ=\Xi$. The only conditions left in Proposition 6.7 are that $*$ is associative and (6-11).

Example 6.9. In the easier case of Example 6.2, we already know the answer: a Poisson-compatible bicovariant flat preconnection on $\tilde{\mathfrak{m}}^{*}=\underline{\mathfrak{m}}>\mathfrak{m}^{*}$ corresponds to a pre-Lie algebra structure on $\tilde{\mathfrak{m}}=\mathfrak{m}^{*} \rtimes \mathfrak{m}$.

Assume o is such a pre-Lie structure, and also assume o is such that $\tilde{o}(\mathfrak{m} \otimes \mathfrak{m}) \subseteq \mathfrak{m}$, $\tilde{o}\left(\mathfrak{m}^{*} \otimes \mathfrak{m}^{*}\right) \subseteq \mathfrak{m}^{*}, \tilde{o}\left(\mathfrak{m} \otimes \mathfrak{m}^{*}\right) \subseteq \mathfrak{m}^{*}$ and that the restriction of $\tilde{o}$ on the other subspace is zero. Directly from the definition of pre-Lie structure, one can show $\circ:=\left.\tilde{o}\right|_{\mathfrak{m} \otimes \mathfrak{m}}$ also provides a pre-Lie structure for $\left(\mathfrak{m},[,]_{\mathfrak{m}}\right)$, while $*:=\left.\tilde{o}\right|_{\mathfrak{m}^{*} \otimes \mathfrak{m}^{*}}$ provides a pre-Lie structure for $\left(\mathfrak{m}^{*},[,]_{\mathfrak{m}^{*}}=0\right)$, thus $*$ is associative and (6-11) holds automatically. Meanwhile, $\triangleright:=\left.\tilde{o}\right|_{\mathfrak{m} \otimes \mathfrak{m}^{*}}$ can be shown to be a left $\mathfrak{m}$-action on $\mathfrak{m}^{*}$, which is exactly the adjoint to the left $\mathfrak{m}$-action on $\mathfrak{m}$ given by the pre-Lie structure $\circ$ on $\mathfrak{m}$. Applying o to any $x \in \mathfrak{m}, \phi, \psi \in \mathfrak{m}^{*}$, one has $x \triangleright(\phi * \psi)=$ $(x \triangleright \phi) * \psi+\phi *(x \triangleright \psi)$, i.e., (6-9). The analysis above shows that $\circ, *, \triangleright$ corresponds to the data in Corollary 6.8. So this example agrees with our construction of Poissoncompatible bicovariant flat preconnections on $\underline{\mathfrak{g}}^{*} \triangleleft \mathfrak{g}=\underline{\mathfrak{m}} \succ \mathfrak{m}^{*}$ in the case of $\mathfrak{g}=\left(\mathfrak{m}^{*},[,]_{\mathfrak{m}^{*}}=0\right)$ in Corollary 6.8.

We already know how to quantise the algebra $C^{\infty}\left(\tilde{\mathfrak{m}}^{*}\right)$ or $S(\tilde{\mathfrak{m}})$ and its differential graded algebra as in Example 4.3. More precisely, the quantisation of $S(\tilde{\mathfrak{m}})$ is the noncommutative algebra $U_{\lambda}(\tilde{\mathfrak{m}})$ with relations $x y-y x=\lambda[x, y]$ for all $x, y \in \tilde{\mathfrak{m}}$, so

$$
U_{\lambda}(\tilde{\mathfrak{m}})=U_{\lambda}\left(\mathfrak{m}^{*} \rtimes \mathfrak{m}\right)=S\left(\mathfrak{m}^{*}\right) \rtimes U_{\lambda}(\mathfrak{m})
$$


with cross relations $x \phi-\phi x=\lambda x \triangleright \phi$ for all $x \in \mathfrak{m}, \phi \in \mathfrak{m}^{*}$. Meanwhile, as in Example 4.3 and Proposition 4.4, the preconnection on $\tilde{\mathfrak{m}}^{*}=\underline{\mathfrak{m}}>\mathfrak{m}^{*}$ is given by

$$
\gamma((\phi, x), \mathrm{d}(\psi, y))=\mathrm{d}((\phi, x) \tilde{o}(\psi, y))=\mathrm{d}(\phi * \psi+x \triangleright \phi, x \circ y) .
$$

Thus, the quantised differential calculus is

$$
\Omega\left(U_{\lambda}(\tilde{\mathfrak{m}})\right)=U_{\lambda}(\tilde{\mathfrak{m}}) \bowtie \Lambda(\tilde{\mathfrak{m}})=\left(S\left(\mathfrak{m}^{*}\right) \rtimes U_{\lambda}(\mathfrak{m})\right) \bowtie \Lambda\left(\mathfrak{m}^{*} \oplus \mathfrak{m}\right)
$$

with bimodule relations

$$
[(\phi, x), \mathrm{d}(\psi, y)]=\lambda \mathrm{d}(\phi * \psi+x \triangleright \phi, x \circ y)
$$

for all $(\phi, x),(\psi, y) \in \tilde{\mathfrak{m}} \subset U_{\lambda}(\tilde{\mathfrak{m}})$, where $\Lambda\left(\mathfrak{m}^{*} \oplus \mathfrak{m}\right)$ denotes the usual exterior algebra on the vector space $\mathfrak{m}^{*} \oplus \mathfrak{m}$ and $\mathrm{d}(\psi, y)=1 \otimes(\psi+y) \in 1 \otimes \Lambda$.

For a concrete example, we take $\mathfrak{m}$ the 2 -dimensional complex nonabelian Lie algebra defined by $[x, y]=x$ and for $\mathfrak{m}^{*}$ the 2-dimensional abelian Lie algebra with its five families of pre-Lie structures [Burde 1998]. Among many choices of pairs of pre-Lie structures for $\mathfrak{m}$ and $\mathfrak{m}^{*}$, there are two pairs which meet our condition (6-9) and provide a pre-Lie structure for $\tilde{\mathfrak{m}}=\mathfrak{m}^{*} \succ \mathfrak{m}$, namely

$$
\begin{aligned}
& y \circ x=-x, \quad y^{2}=-\frac{1}{2} y, \quad Y * Y=X, \\
& x \triangleright X=0, \quad x \triangleright Y=0, \quad y \triangleright X=X, \quad y \triangleright Y=\frac{1}{2} Y \text {; } \\
& y \circ x=-x, \quad X * Y=X, \quad Y * X=X, \quad Y * Y=Y, \\
& x \triangleright X=0, \quad y \triangleright Y=0, \quad y \triangleright X=X, \quad y \triangleright Y=0,
\end{aligned}
$$

where $\{X, Y\}$ is chosen to be the basis of $\mathfrak{m}^{*}$ dual to $\{x, y\}$. By Theorem 6.6 and the general analysis earlier, we know that $\Omega\left(U_{\lambda}(\tilde{\mathfrak{m}})\right)=U_{\lambda}(\tilde{\mathfrak{m}}) \ltimes \Lambda\left(\mathfrak{m}^{*} \oplus \mathfrak{m}\right)$ is a bicovariant differential graded algebra. In particular,

$$
\Omega^{1}\left(U_{\lambda}(\tilde{\mathfrak{m}})\right)=U_{\lambda}(\tilde{\mathfrak{m}}) \mathrm{d} x \oplus U_{\lambda}(\tilde{\mathfrak{m}}) \mathrm{d} y \oplus U_{\lambda}(\tilde{\mathfrak{m}}) \mathrm{d} X \oplus U_{\lambda}(\tilde{\mathfrak{m}}) \mathrm{d} Y .
$$

The bimodule relations for case (1) are

$$
\begin{gathered}
{[y, \mathrm{~d} x]=-\lambda \mathrm{d} x, \quad[y, \mathrm{~d} y]=-\frac{1}{2} \lambda \mathrm{d} y, \quad[Y, \mathrm{~d} Y]=\lambda \mathrm{d} X,} \\
{[y, \mathrm{~d} X]=\lambda \mathrm{d} X, \quad[y, \mathrm{~d} Y]=\frac{1}{2} \lambda \mathrm{d} Y .}
\end{gathered}
$$

For case (2), we have

$$
\begin{gathered}
{[y, \mathrm{~d} x]=-\lambda \mathrm{d} x, \quad[X, \mathrm{~d} Y]=\lambda \mathrm{d} X, \quad[Y, \mathrm{~d} X]=\lambda \mathrm{d} X, \quad[Y, \mathrm{~d} Y]=\lambda \mathrm{d} Y,} \\
{[y, \mathrm{~d} X]=\lambda \mathrm{d} X .}
\end{gathered}
$$

Example 6.10. Suppose that $\mathfrak{g}$ is quasitriangular with $r_{+} \triangleright x=0$ for all $x \in \mathfrak{g}$ as in Example 6.3. According to Corollary 6.8, if $\mathfrak{g}$ admits a pre-Lie product $*$ such that

$$
\left[r^{(1)}, x * y\right] \otimes r^{(2)}=\left[r^{(1)}, x\right] * y \otimes r^{(2)}+x *\left[r^{(1)}, y\right] \otimes r^{(2)},
$$


from (6-9), then $\overline{\mathfrak{g}} \rtimes \mathfrak{g}^{*}$ in Example 6.3 admits a pre-Lie structure õ

$$
x \tilde{\circ} y=x * y, \quad \phi \tilde{\circ} x=\phi \triangleright x=-\left\langle\phi, r^{(2)}\right\rangle\left[r^{(1)}, x\right], \quad \phi \tilde{\circ} \psi=-\left\langle\phi, r^{(2)}\right\rangle \operatorname{ad}_{r^{(1)}}^{*} \psi,
$$

and thus determines a Poisson-compatible left-covariant flat preconnection on the cotangent bundle $\mathfrak{g}^{*} \rtimes G$. Such a preconnection is bicovariant if $*$ is associative and (6-11) holds, and in this case condition (6-9) vanishes. Recall that we cannot take $\mathfrak{g}$ semisimple here since it will not then admit a pre-Lie structure.

For a concrete example, we take $\mathfrak{g}$ to again be the 2-dimensional Lie algebra $[x, t]=x$ as in Example 4.5 but with $\delta x=0$ and $\delta t=x \otimes t-t \otimes x$ as a triangular Lie bialgebra with $r=t \otimes x-x \otimes t$. If $\{X, T\}$ is the dual basis to $\{x, t\}$ then the pre-Lie algebra structure $\circ$ of $\mathfrak{g}^{*}$ determined by $r$ is

$$
T \circ X=-T, \quad X \circ X=-X,
$$

and otherwise zero, which is isomorphic to $\mathfrak{b}_{2,1}$ listed in Example 4.5. On the other hand, computation shows that among all the possible pre-Lie algebra structures for $\mathfrak{g}$ listed in Example 4.5, precisely $\mathfrak{b}_{1,-1}$ and $\mathfrak{b}_{2,1}$ satisfy condition (6-14), giving us two pre-Lie algebra structures on $\overline{\mathfrak{g}} \rtimes \mathfrak{g}^{*}$ by our construction, namely

$$
\begin{aligned}
& t * x=-x, \quad t * t=-t, \quad T \circ X=-T, \quad X \circ X=-X, \\
& X \triangleright x=x, \quad T \triangleright t=x ; \\
& x * t=x, \quad t * t=t, \quad T \circ x=-T, \quad X \circ X=-X, \\
& X \triangleright x=x, \quad T \triangleright t=x .
\end{aligned}
$$

These determine two Poisson-compatible left-covariant flat preconnections on the cotangent bundle $\mathfrak{g}^{*} \triangleleft G$. In case (1) this is also bicovariant as $*$ is associative and satisfies (6-11), which can be checked directly.

\section{References}

[Beggs and Majid 2006] E. J. Beggs and S. Majid, "Semiclassical differential structures", Pacific J. Math. 224:1 (2006), 1-44. MR Zbl

[Beggs and Majid 2010] E. J. Beggs and S. Majid, "Quantization by cochain twists and nonassociative differentials", J. Math. Phys. 51:5 (2010), art. ID 053522. MR Zbl

[Beggs and Majid 2014a] E. J. Beggs and S. Majid, "Gravity induced from quantum spacetime", Classical Quantum Gravity 31:3 (2014), art. ID 035020. MR Zbl

[Beggs and Majid 2014b] E. J. Beggs and S. Majid, "Semiquantisation functor and Poisson-Riemannian geometry, I", preprint, 2014. arXiv

[Burde 1994] D. Burde, "Left-symmetric structures on simple modular Lie algebras", J. Algebra 169:1 (1994), 112-138. MR Zbl

[Burde 1998] D. Burde, "Simple left-symmetric algebras with solvable Lie algebra", Manuscripta Math. 95:3 (1998), 397-411. MR Zbl 
[Burde 2006] D. Burde, "Left-symmetric algebras, or pre-Lie algebras in geometry and physics", Cent. Eur. J. Math. 4:3 (2006), 323-357. MR Zbl

[Cartier 2009] P. Cartier, "Vinberg algebras and combinatorics", preprint (IHES/M/09/34), Institut des Hautes Études Scientifiques, 2009, available at http://goo.gl/H74Hzz.

[Drinfeld 1987] V. G. Drinfeld, "Quantum groups”, pp. 798-820 in Proceedings of the International Congress of Mathematicians (Berkeley, CA, 1986), vol. 1, edited by A. M. Gleason, Amer. Math. Soc., Providence, RI, 1987. MR Zbl

[Hawkins 2004] E. Hawkins, "Noncommutative rigidity", Comm. Math. Phys. 246:2 (2004), 211-235. MR Zbl

[Huebschmann 1990] J. Huebschmann, "Poisson cohomology and quantization", J. Reine Angew. Math. 408 (1990), 57-113. MR Zbl

[Majid 1990a] S. Majid, "Matched pairs of Lie groups associated to solutions of the Yang-Baxter equations", Pacific J. Math. 141:2 (1990), 311-332. MR Zbl

[Majid 1990b] S. Majid, "Physics for algebraists: noncommutative and noncocommutative Hopf algebras by a bicrossproduct construction", J. Algebra 130:1 (1990), 17-64. MR Zbl

[Majid 1995] S. Majid, Foundations of quantum group theory, Cambridge University Press, 1995. MR Zbl

[Majid 2000] S. Majid, "Braided-Lie bialgebras", Pacific J. Math. 192:2 (2000), 329-356. MR Zbl

[Majid and Tao 2015a] S. Majid and W.-Q. Tao, "Cosmological constant from quantum spacetime", Phys. Rev. D 91:12 (2015), 124028, 10. MR

[Majid and Tao 2015b] S. Majid and W.-Q. Tao, "Duality for generalised differentials on quantum groups", J. Algebra 439 (2015), 67-109. MR Zbl

[Meljanac et al. 2012] S. Meljanac, S. Krešić-Jurić, and R. Štrajn, "Differential algebras on $\kappa$ Minkowski space and action of the Lorentz algebra", Internat. J. Modern Phys. A 27:10 (2012), art. ID 1250057. MR Zbl

[Woronowicz 1989] S. L. Woronowicz, "Differential calculus on compact matrix pseudogroups (quantum groups)", Comm. Math. Phys. 122:1 (1989), 125-170. MR Zbl

Received February 5, 2015. Revised March 3, 2016.

\section{SHAHN MAJID}

SCHOOL OF Mathematical SCIENCES

QUEEN MARY UNIVERSITY OF LONDON

MILE END ROAD

LONDON

E1 4NS

UNITED KINGDOM

s.majid@qmul.ac.uk

WEN-QING TAO

SCHOOL OF MATHEMATICS AND STATISTICS

HUAZHONG UNIVERSITY OF SCIENCE AND TECHNOLOGY

WUHAN, 430074

CHINA

wqtao@ hust.edu.cn 


\title{
PACIFIC JOURNAL OF MATHEMATICS
}

Founded in 1951 by E. F. Beckenbach (1906-1982) and F. Wolf (1904-1989)

$$
\text { msp.org/pjm }
$$

\section{EDITORS}

\author{
Don Blasius (Managing Editor) \\ Department of Mathematics \\ University of California \\ Los Angeles, CA 90095-1555 \\ blasius@math.ucla.edu
}

\author{
Paul Balmer \\ Department of Mathematics \\ University of California \\ Los Angeles, CA 90095-1555 \\ balmer@math.ucla.edu \\ Robert Finn \\ Department of Mathematics \\ Stanford University \\ Stanford, CA 94305-2125 \\ finn@math.stanford.edu \\ Sorin Popa \\ Department of Mathematics \\ University of California \\ Los Angeles, CA 90095-1555 \\ popa@math.ucla.edu
}

\author{
Vyjayanthi Chari \\ Department of Mathematics \\ University of California \\ Riverside, CA 92521-0135 \\ chari@math.ucr.edu \\ Kefeng Liu \\ Department of Mathematics \\ University of California \\ Los Angeles, CA 90095-1555 \\ liu@math.ucla.edu \\ Igor Pak \\ Department of Mathematics \\ University of California \\ Los Angeles, CA 90095-1555 \\ pak.pjm@gmail.com \\ Paul Yang \\ Department of Mathematics \\ Princeton University \\ Princeton NJ 08544-1000 \\ yang@math.princeton.edu
}

\section{PRODUCTION}

Silvio Levy, Scientific Editor, production@msp.org

\section{SUPPORTING INSTITUTIONS}

ACADEMIA SINICA, TAIPEI

CALIFORNIA INST. OF TECHNOLOGY

STANFORD UNIVERSITY

UNIV. OF BRITISH COLUMBIA

UNIV. OF CALIFORNIA, BERKELEY

UNIV. OF CALIFORNIA, DAVIS

UNIV. OF CALIFORNIA, LOS ANGELES

UNIV. OF CALIFORNIA, RIVERSIDE

UNIV. OF CALIFORNIA, SAN DIEGO

UNIV. OF CALIF., SANTA BARBARA
KEIO UNIVERSITY

MATH. SCIENCES RESEARCH INSTITUTE

NEW MEXICO STATE UNIV.

OREGON STATE UNIV.
Daryl Cooper

Department of Mathematics

University of California

Santa Barbara, CA 93106-3080 cooper@math.ucsb.edu

Jiang-Hua Lu

Department of Mathematics

The University of Hong Kong

Pokfulam Rd., Hong Kong

jhlu@maths.hku.hk

$$
\text { Jie Qing }
$$

Department of Mathematics

University of California

Santa Cruz, CA 95064

qing@ cats.ucsc.edu

\author{
UNIV. OF CALIF., SANTA CRUZ \\ UNIV. OF MONTANA \\ UNIV. OF OREGON \\ UNIV. OF SOUTHERN CALIFORNIA \\ UNIV. OF UTAH \\ UNIV. OF WASHINGTON \\ WASHINGTON STATE UNIVERSITY
}

These supporting institutions contribute to the cost of publication of this Journal, but they are not owners or publishers and have no responsibility for its contents or policies.

See inside back cover or msp.org/pjm for submission instructions.

The subscription price for 2016 is US $\$ 440 /$ year for the electronic version, and \$600/year for print and electronic.

Subscriptions, requests for back issues and changes of subscriber address should be sent to Pacific Journal of Mathematics, P.O. Box 4163, Berkeley, CA 94704-0163, U.S.A. The Pacific Journal of Mathematics is indexed by Mathematical Reviews, Zentralblatt MATH, PASCAL CNRS Index, Referativnyi Zhurnal, Current Mathematical Publications and Web of Knowledge (Science Citation Index).

The Pacific Journal of Mathematics (ISSN 0030-8730) at the University of California, c/o Department of Mathematics, 798 Evans Hall \#3840, Berkeley, CA 94720-3840, is published twelve times a year. Periodical rate postage paid at Berkeley, CA 94704, and additional mailing offices. POSTMASTER: send address changes to Pacific Journal of Mathematics, P.O. Box 4163, Berkeley, CA 94704-0163.

PJM peer review and production are managed by EditFLOW ${ }^{\circledR}$ from Mathematical Sciences Publishers.

PUBLISHED BY

\section{I. mathematical sciences publishers}

nonprofit scientific publishing

http://msp.org/

(C) 2016 Mathematical Sciences Publishers 


\section{PACIFIC JOURNAL OF MATHEMATICS}

Volume $284 \quad$ No. $1 \quad$ September 2016

Bitwist manifolds and two-bridge knots

JAmes W. CANNON, William J. Floyd, LEeR LAMbert,

WALTER R. PARry and Jessica S. PurCELL

Recognizing right-angled Coxeter groups using involutions

Charles Cunningham, Andy Eisenberg, Adam Piggott and KIM RUANE

On Yamabe-type problems on Riemannian manifolds with boundary

Marco Ghimenti, Anna Maria Micheletti and Angela

PISTOIA

Quantifying separability in virtually special groups

MARK F. HAGEN and PRIYAM PATEL

Conformal designs and minimal conformal weight spaces of vertex operator superalgebras

TOMONORI HASHIKAWA

Coaction functors

S. KALiszewski, Magnus B. LANDSTAD and John QuigG

Cohomology and extensions of braces

VICTORIA LEBED and LEANDRO VENDRAMIN

Noncommutative differentials on Poisson-Lie groups and pre-Lie algebras

SHAHN MAJID and WEN-QING TAO 